\title{
Unconventional Superconductivity in Samarium Nitride
}

By

\section{William Doonan}

\author{
A thesis \\ submitted to the Victoria University of Wellington \\ in fulfilment of the requirements for the degree of \\ Master of Science
}

Victoria University of Wellington 2020 


\begin{abstract}
We have studied the nature of unconventional superconductivity in the rare-earth nitride (REN) samarium nitride ( $\mathrm{SmN}$ ) for the purposes of providing a deeper understanding of the mechanisms that lead to such a phenomenon in an already extremely interesting material. An approximate low energy model has been introduced for $\mathrm{SmN}$ based on previous bandstructure calculation and recent experimental results. This consists of the non-dispersive $4 f$ band associated with the samarium ion crossing through the dispersive $5 d$ band associated with the nitrogen ion. Due to large spin polarisation in the bandstructure we need only consider the majority-spin $5 d$ and $4 f$ bands which lead to an essentially spinless two band system. Starting from this two band system, we apply the $\mathbf{k} \cdot \mathbf{p}$ method to it in order to create an effective model for the system. This effective model for the material acts as the platform from which we study the possible triplet superconducting pairing. Basing our pairing on the electron-phonon interaction we have postulated the existence of triplet pairing in the $5 d$ band, from which we have successfully characterised the pair potential in this system through the self-consistency equation. The pair potential $\Delta_{d}$ could be solved analytically in a special case where the Fermi level was equal to the $4 f$ band. In this case we find that above a threshold effective coupling strength the superconducting state is established and analytically known. In contrast to this result for the more general case where the Fermi level is different to the $4 f$ band we numerically recover a solution that was exponential in the effective coupling strength which is similar to the pairing as we expect from the single band case. Analytic solutions in this case were not able to be found, however, we know that from our numerical investigations there will exist a solution for any effective coupling strength, contrasting with the special case where the pairing amplitude can disappear below a certain threshold. In conjunction to these results we also examined the situation where the $5 d$ and $4 f$ bands have hybridised together in order to search for unique pairing that may be resistant to disorder. By keeping the triplet pairing only in the $5 d$ band, this translates to hybrid pairing between electrons in the two hybridised bands. Results from the hybridised bands system show a new singlet-like pairing $\Delta_{\mathcal{S}}$ which is even in $\mathbf{k}$ and singlet in the hybridised band indices. Preliminary numerical results suggest that this pairing indeed exists and occurs only near the avoided crossing of the hybridised bands. The existence of such a pairing, originating from triplet pairing, has exciting implications for the robustness of the superconductivity in the presence of disorder and/or impurities.
\end{abstract}




\section{Acknowledgements}

I would like to thank my two supervisors; Michele and Uli, without whom I would never have been able to complete this study. Their tireless patience for questions and their physical insight have taught me much about our world, as well as how to stay positive in the face of seemingly insurmountable problems.

I gratefully thank Victoria University of WELLINGTON and the MacDiarmid Institute for financial assistance for my study.

Finally I thank my family for their constant love and support, which has allowed me to wholeheartedly work towards my aspirations. 


\section{Contents}

Abstract

Acknowledgements

1 Introduction $\quad 1$

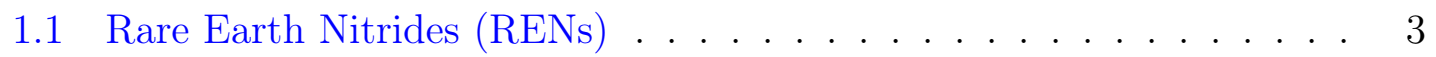

1.1.1 Properties of Samarium Nitride $(\mathrm{SmN})$. . . . . . . . . . . 4

1.2 Superconductivty . . . . . . . . . . . . . . . 6

1.2.1 BCS Superconductivity . . . . . . . . . . . . 7

1.2.2 Unconventional Superconductivity . . . . . . . . . . . . . 9

$1.2 .3 p$-wave Superconductivity . . . . . . . . . . . . . . . 13

1.3 The $\mathbf{k} \cdot \mathbf{P}$ Method . . . . . . . . . . . . . . . . 15

1.4 Outline . . . . . . . . . . . . . . . . 16

2 The Model $\quad 19$

2.1 Band-Structure of $\mathrm{SmN} \ldots \ldots$. . . . . . . . . . . . . . . . 19

2.2 The Effective Model of $\mathrm{SmN}$. . . . . . . . . . . . . . . . . . 22

2.3 The Electron-Phonon Interaction . . . . . . . . . . . . . . 26

3 Triplet Pairing Superconductivity in SmN 31

3.1 The Effective Hamiltonian . . . . . . . . . . . . . . . . . . . 31

3.2 The Mean Field Hamiltonian _. . . . . . . . . . . . . . . . . . 34

3.2.1 Diagonalising the Mean-Field Hamiltonian . . . . . . . . . . . 37

3.3 Self Consistency Equation for $\Delta_{d} \ldots \ldots$. . . . . . . . . . 39

3.3.1 Treating the Self Consistency Integral . . . . . . . . . . . . . 41

3.4 Results . . . . . . . . . . . . . . . . . . . . 45

3.4.1 Special Case When $\varepsilon_{f b}=0 \ldots \ldots$. . . . . . . . 45

3.4 .2 General Case With $\varepsilon_{f b} \neq 0 \ldots \ldots$. . . . . . . . 55

3.4.3 General Case; Small $\left|\Delta_{d}\right| \ldots \ldots \ldots$. . . . . . . . . 57

3.5 Conclusions . . . . . . . . . . . . . . . . . . . . 60 
4 Pairing Between Mixed Bands $\quad 63$

4.1 The Effective Hamiltonian for the Mixed Bands . . . . . . . . . . . . 63

4.2 The Mean Field Hamiltonian . . . . . . . . . . . . . . . . . 66

4.2.1 Emergence of Even Parity Pairing . . . . . . . . . . . . . 70

4.3 The Self-Consistency Equation . . . . . . . . . . . . . . . . . . 72

4.3.1 Treating the Self-Consistency Equation . . . . . . . . . . . 73

4.4 Results . . . . . . . . . . . . . . . . 76

4.5 Conclusions . . . . . . . . . . . . . . . . . . . 78

5 Summary, Conclusions, and Outlook $\quad 79$

$\begin{array}{ll}\text { Appendices } & 83\end{array}$

A Simplification of the Expectation Value $\quad 85$

B Treating $\sin 2 \alpha(\mathbf{k}) \quad \mathbf{8 9}$ 


\section{Chapter 1}

\section{Introduction}

In 1965 Gordan Moore [1] made his stunning observation that every two years or so the number of transistors that fit on a silicon chip doubled. This is commonly known as Moore's Law and it has ushered in a technological boom, the likes of which the world hasn't seen since the start of the industrial age. However, as we start to see the saturation of Moore's Law [2] we must move past the miniaturisation of existing technologies and look towards the future of new and exciting information technologies. Such examples include spintronic devices, molecular electronics, and of course the fabled quantum computer. Looking towards this future requires a deep and thorough understanding of new and exotic materials to push into this new frontier and to make these technologies a reality.

The end of Moore's law is inevitable, of course, due to size constraints on a physical transistor and the new possibility of transistors being manufactured on such a small scale that quantum effects start to manifest in the transistor [3]. However, a more pressing issue for the construction of super dense computer chips is the amount of heat they radiate collectively and the amount of damage that will do to the chip itself. If not cooled properly, new chips cannot perform to their optimal performance since current supplied to the chip will need to be throttled so as not to threaten the integrity of the chip. With rising costs of cooling such chips, businesses are hesitating to invest in the development of smaller transistors. To continue the growth of the sector we may look to spintronic devices which use the manipulation of an electron's spin. These devices would move the current industry away from the silicon transistor towards devices that use the small magnetic moment of electrons, rather than their charge. As a result these devices have been predicted to be smaller, faster, and more powerful than their electric cousins. If we were to look at a spintronic transistor instead of the common electric one, the amount of current required to operate such a device will be smaller since the spintronic device can be 
manipulated with a small magnetic field, rather than a comparably larger current. This would protect the integrity of the chip at higher temperatures as well as more punishing environments and in higher densities. As well as this, the promise of high-temperature superconductivity could also move the industry forward. If the chip or internal circuitry of the transistor was fashioned from such a material then there would be no heat generated by the internal resistance of the circuitry, limiting or even eradicating such wayward heat from damaging the chip. Current materials that have promise for spintronic devices are the rare-earth nitrides (RENs) due to their strong and unique magnetic properties as well as our material of interest, samarium nitride $(\mathrm{SmN})$, which has been shown to be superconducting as well as a semiconductor [4-9]. The understanding of these materials may be the key to realising some of these exciting new ideas.

The rare-earth nitrides were first investigated in the 1960s with much promise for new technological applications however not much progress was made due to poor stoichiometry of samples which is impacted by the materials' ready oxidation in ambient conditions $[10,11]$. Recently however progress on the RENs has grown rapidly due to breakthroughs in the production of high quality epitaxial thin films [9, 12-16]. Some RENs have exhibited semiconducting and ferromagnetic properties [6, $12,14,17-20]$ which has potential for exploitation of the spin of charge carriers in semiconducting technologies as discussed above. There are now about a dozen laboratories worldwide reporting growth and study of REN thin films [4].

Samarium nitride stands out from the rest of the RENs since has been reported to be a low-moment ferromagnetic semiconductor $[6-9,21]$ which is already interesting enough for potential spintronic devices. However, it is with great interest that $\mathrm{SmN}$ has been observed to be superconducting. The mechanism behind the superconductivity in $\mathrm{SmN}$ is believed to be based on $p$-wave superconductivity due to the spin polarization of its bands [22]. However nothing in depth is known theoretically about the phenomenon in $\mathrm{SmN}$ and the development of such knowledge may lead to improvements in its critical temperature for viable use in industry. This system may also inspire the creation of some new material that will lead to commercialisation. Therefore, in this thesis we will be looking to describe the superconductivity of SmN in fuller detail than has been done before. We will use an idealised model of SmN's bandstructure to study the effect of possible $p$-wave superconducting pairing in the region where two energy bands cross close to the Fermi-energy. The existence of such multi-band $p$-wave pairing is intriguing and may be the reason as to why we see superconductivity at all in this system. 


\subsection{Rare Earth Nitrides (RENs)}

Rare earth nitrides (RENs) are materials that have one of the elements from the Lanthanide series (from $57(\mathrm{La})$ to $71(\mathrm{Lu})$ ) bonded to nitrogen. The RENs we will consider are the so called rare earth mononitides where the rare-earth forms an $\mathrm{RE}^{3+}$ cation and bonds to a single nitrogen $\mathrm{N}^{3-}$ anion. RENs show promise in a wide variety of applications such as the realisation of spintronic devices, infrared (IR) detectors and as contacts to group III-V compounds [4].

The rare-earths of the Lanthanide series are intriguing as they are the elements that have increasingly filled $4 f$ orbitals. They have atomic configurations of $[\mathrm{Xe}] 6 s^{2} 4 f^{n}$ with $n$ ranging from $0(\mathrm{La})$ to $14(\mathrm{Lu})$ with some elements (La, Ce, $\mathrm{Gd}, \mathrm{Lu}$ ) having extra $5 d$ electrons. The $4 f$ states that make these elements interesting are very localised and are often called heavy-fermions due to their localisation and characteristically flat band dispersions. The highly localised nature of the $4 f$ electrons leads to the electrons having atomic-like properties and heavily influences the material's strong magnetic properties. The rare-earths are also the only stable elements with significant filling of the $4 f$ shell which give them their large spin and orbital moments, making them desirable for applications that involve large moments such as spintronic devices.

The RENs all form in the face-centered cubic (FCC) NaCl structure, see figure 1.1, with lattice constants ranging from $5.305 \AA$ for $\mathrm{LaN}$ [23] to $4.76 \AA$ for LuN [24]. This structure is also shared by the rare earth pnictides (RE-Vs), of which Nitrogen is the first pnictide. This structure has made the RE-Vs useful for theoretical calculations due its simple crystal structure. Many studies have tried to use these materials to better understand how the localised and strongly correlated $4 f$ electrons alter a material's bandstructure. The interactions of these $4 f$ electrons with a material's bandstructure have been modelled using a variety of different methods [22, 25-44] to varying degrees of success. The RENs also have a strong exchange interaction which induces large spin splitting of the energy bands for both the conduction and valence bands. The majority spin has higher energy in the valence band and lower energy in the conduction band which means that at low temperatures charge carriers (either electrons or holes) will all be of the majority spin (minority-spin bands are unoccupied at lower temperatures [4]).

The strong magnetic behaviour exhibited in the rare-earths is also seen on display in the RENs. An example of this is that almost all RENs are ferromagnetic [45-47]. They only adopt magnetic order at low temperatures however, the highest of which is the Curie temperature $\left(T_{C}\right)$ of $\operatorname{GdN}(70 \mathrm{~K})[48,49]$. These magnetic properties are offshoots of the $4 f$ shell electrons, as stated before. The magnetic properties of 


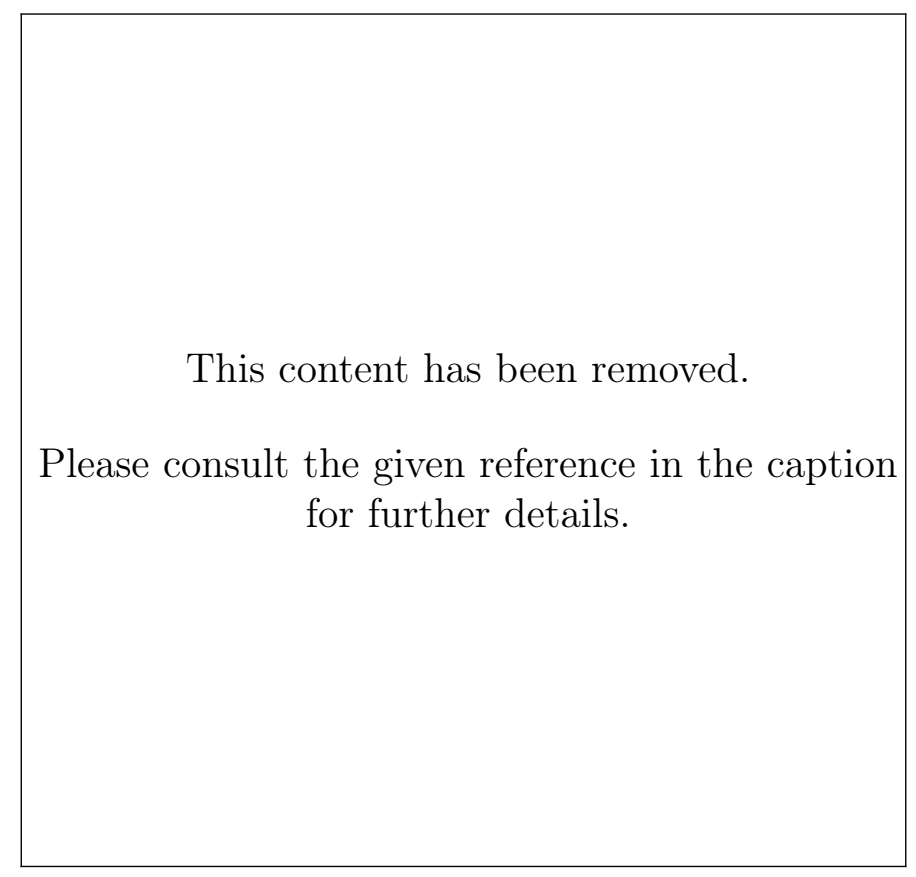

Figure 1.1: The $\mathrm{NaCl}$ structure of the RENs. The large spheres represent the rare earth cation while the small spheres represent the Nitrogen anion. Picture taken from [4] (Figure 1).

the RENs can be roughly understood using Hund's rules and in fact leads to the breaking of cubic crystal symmetry [22] in the materials. This is due to a non-zero orbital angular momentum in all the rare-earths, excepting Gd (see figure 1.2).

\subsubsection{Properties of Samarium Nitride $(\mathrm{SmN})$}

Samarium Nitride $(\mathrm{SmN})$ is the REN that uses the rare earth Samarium (Sm) which has an atomic structure [Xe] $6 s^{2} 4 f^{5}$. It has five filled $4 f$ states and 9 unfilled $4 f$ states, one of which is close to the Fermi level. This one low-lying unfilled $4 f$ state is unusual for a REN since photoemission and inverse photoemission show that the $4 f$ shells are in most cases well below (filled) or above (empty) the Fermi level $[22,50,51]$. This leads to interesting structure near the Fermi level which will be the basis of our investigation.

Samarium has a curious magnetic structure due to its number of $4 f$ electrons. Based on Hund's rules we expect that the spin and orbital moments of Sm to cancel each other out, giving a net zero total angular momentum $J$ as seen in figure 1.2. This property carries over to SmN where Preston et al. [6] have measured SmN to have a very small moments, less than $0.1 \mu_{B}$ per Sm ion even in a $6 T$ applied field at low temperatures. Due to the large spin-splitting in its band dispersions (fig 2.3 and 2.4) it becomes a material with single-spin transport with a small magnetic 


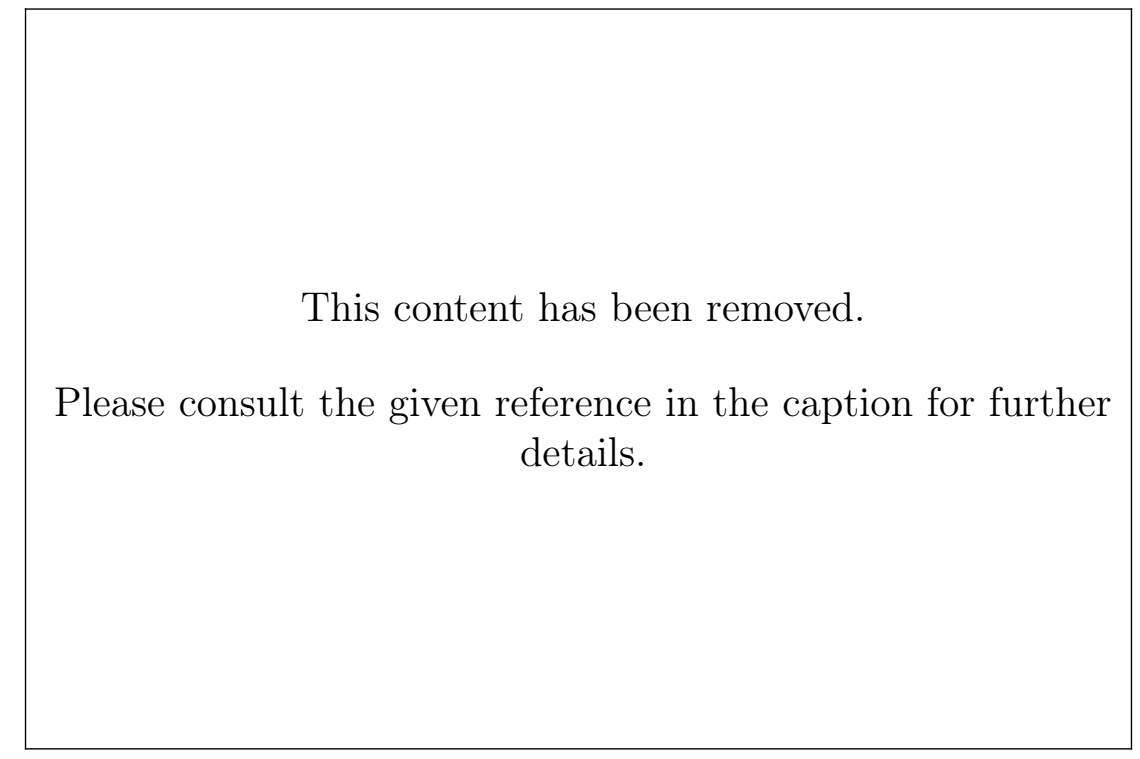

Figure 1.2: The magnetic moments of all the RENs decomposed into spin and orbital contributions based on a Hund's rule density matrix [22]. SmN is predicted to have a close to zero total magnetic moment which has been experimentally verified $[6,7,21]$. Figure taken from [22] (Figure 5(c)).

moment. This is because the only bands available for low energy transport near the Fermi energy will occur in the majority spin bands. Along with its small moment $\mathrm{SmN}$ is a ferromagnetic material at low temperatures with a $T_{C}$ of around $27 \mathrm{~K}$. Coupled to this is the experimental evidence for $\mathrm{SmN}$ being a semi-conductor [4-9], as compared to being a semi-metal as previously predicted [30]. This allows it to have exciting potential in spintronic devices, for example it could be paired with another spin dependent material with a large moment (say GdN) to create a spindependent memory element, as well as having the advantage of being able to inject spin-polarized electrons into a conventional semiconductor without the deleterious effects of a fringe magnetic field [52].

$\mathrm{SmN}$ has also recently been reported to be superconductive [5] with critical temperatures of less than $5 K$. This is an unexpected phenomenon to observe in $\mathrm{SmN}$ since superconductivity is generally due to the common superconducting pairing, $s$-wave pairing, involving both spin up and spin down electrons. This is impossible at low energies for $\mathrm{SmN}$ due to its spin polarization which means that that the superconducting pairing must pair together electrons of the same spin. Because of the large spin-splitting and subsequent spin polarization, $\mathrm{SmN}$ opens the door to unconventional superconducting pairing present in the material, specifically tripletpairing in this case. While the coexistence of magnetism and superconductivity in bulk samples is not unheard of [53-55] what makes $\mathrm{SmN}$ special is that its superconductivity must likely be based on the heavy fermion electrons in the $4 f$ band [5]. The 
necessity of these heavy fermions is so the triplet-pairing may survive in the presence of disorder in the crystal, which has been established to severely limit the critical temperature of such superconductors $[56,57]$. This allows $\mathrm{SmN}$ to become a versatile tool to access new states of quantum matter [58] due to its interplay between its low moment ferromagnetism, superconductivity, and semi-conductor nature. To first do this, a fuller understanding is required of the superconductivity at play in $\mathrm{SmN}$ which will lead to accessing some of its promise as well as pave the way towards understanding other potential new and exotic superconducting systems.

\section{$1.2 \quad$ Superconductivty}

Superconductivity is the phenomenon whereby a material loses all electrical resistance below a critical temperature and magnetic field. In conventional superconductors there is also no internal magnetic field however, as stated before, there have been reported superconductors where superconductivity and magnetic behaviour have occurred together. This phenomenon was first discovered by Heike Kamerlingh Onnes in 1911 [59] and the first successful microscopic theory to describe the phenomenon was developed by Bardeen, Cooper, and Schrieffer and now called the BCS theory of superconductivity [60]. Superconductors that obey BCS theory are often called BCS superconductors and these materials generally have low critical temperatures $(<30 \mathrm{~K})$ (see figure 1.4). However breakthroughs in new material synthesis have increasingly pushed the upper limit of superconductors critical temperature with some even having critical temperatures up to $138 \mathrm{~K}$ [61] in ambient pressure! Such materials with high critical temperatures may pave the way to innovate new circuitry designs that take advantage of zero electrical resistances and the resulting energy savings and efficiency increases. These materials that push the boundaries, however, are generally not described by the classic BCS framework, a notable exception being $\mathrm{H}_{2} \mathrm{~S}$, and are described instead by processes not fully understood which are all labelled as unconventional superconductivity. Of these unconventional superconductivity mechanisms, triplet pairing is the type we are interested in for $\mathrm{SmN}$ due to its fully spin-polarized conduction bands. Thus understanding these new types of materials will be paramount in the pursuit of engineering new and improved materials to propel us to higher temperature superconductors and new exotic circuitry possibilities. 


\subsubsection{BCS Superconductivity}

The BCS framework, as developed in 1957, involves electrons forming pairs in the presence of a weak interaction which is commonly taken to be the electron-phonon interaction. These pairs are called Cooper pairs after Cooper showed in 1956 that at least one such pair would form in a Fermi sea, so long as the interaction was positive [62]. These pairs form the new ground state of the system and are formed from electrons that have opposite spin and momenta. Using a mean-field approach of the system they found that the energy spectrum of the system became gapped, i.e. they found the existence of an energy region where there could be no quasi-particle excitations. The only states that can exist within this gap is the ground state of the system which, in this instance, are the Cooper pairs. This region is labelled the superconducting gap and usually denoted by $\Delta$. This gap is temperature dependant and at a critical temperature the gap closes to zero with larger temperatures keeping it closed and lower temperatures opening it up. At larger energies there are allowed quasiparticle states but at low enough energies the only particles that will contribute to transport are the allowed Cooper pairs, which leads to the observed phenomenon of zero resistance.

As an introduction to the formalism we start with the well known BCS Hamiltonian

$$
H_{B C S}=\sum_{\mathbf{k}, \sigma} \varepsilon_{\mathbf{k}} c_{\mathbf{k}, \sigma}^{\dagger} c_{\mathbf{k}, \sigma}-V \sum_{\mathbf{k}, \mathbf{k}^{\prime}} c_{\mathbf{k}, \uparrow}^{\dagger} c_{-\mathbf{k}, \downarrow}^{\dagger} c_{-\mathbf{k}^{\prime}, \downarrow} c_{\mathbf{k}^{\prime}, \uparrow}
$$

where $\varepsilon_{\mathbf{k}}$ is the energy dispersion of an electron with momenta $\mathbf{k}, V$ is the effective interaction strength, $\sigma$ is the spin of the electron (either $\uparrow$ or $\downarrow$ ), and $c_{\mathbf{k}, \sigma}^{(\dagger)}$ is the annihilation (creation) operator for an electron with momentum $\mathbf{k}$ and spin $\sigma$. This Hamiltonian can be split into two parts; the first of which describes the energy of the single particle state $|\mathbf{k}, \sigma\rangle$ and the second the effective attractive interaction which is mediated by phonons. This interaction reads: through the absorption (or emission) of a phonon two electrons of states $\left|\mathbf{k}^{\prime}, \uparrow\right\rangle$ and $\left|-\mathbf{k}^{\prime}, \downarrow\right\rangle$ change to the states $|\mathbf{k}, \uparrow\rangle$ and $|-\mathbf{k}, \downarrow\rangle$ thereby pairing the electrons into the Cooper pair state $|\mathbf{k} \uparrow ;-\mathbf{k} \downarrow\rangle$. The interaction is attractive by the negative sign in the Hamiltonian and the assertion that the strength $V>0$. This interaction strength is assumed to be non-vanishing for states in a shell of width $2 \hbar \omega_{D}$ around the Fermi energy, where $\omega_{D}$ is the Debye frequency. The established way to proceed now is to make a mean-field approximation. While the Hamiltonian (1.1) conserves particle numbers, the mean-field Hamiltonian will not. However, the ground state $\left|\phi_{B C S}\right\rangle$ is expected to contain a large number of pairs so we may make the mean-field approximation by expanding in small fluctuations about a mean value of the operators. In such a 
way we may make the definition

$$
\Delta=V \sum_{\mathbf{k}}\left\langle\phi_{B C S}\left|c_{-\mathbf{k}, \downarrow} c_{\mathbf{k}, \uparrow}\right| \phi_{B C S}\right\rangle
$$

which allows us to write the mean-field approximation

$$
V \sum_{\mathbf{k}} c_{-\mathbf{k}, \downarrow} c_{\mathbf{k}, \uparrow}=\Delta+\left(V \sum_{\mathbf{k}} c_{-\mathbf{k}, \downarrow} c_{\mathbf{k}, \uparrow}-\Delta\right)
$$

where the first term is the mean value of the operators and the term in brackets are the small fluctuations about the mean value. Combining this result with the original Hamiltonian (1.1), ignoring constant terms, and measuring the energy dispersions with respect to the chemical potential $\mu$ we come to the mean-field Hamiltonian:

$$
H_{B C S}^{M F}=\sum_{\mathbf{k}, \sigma}\left(\varepsilon_{\mathbf{k}}-\mu\right) c_{\mathbf{k}, \sigma}^{\dagger} c_{\mathbf{k}, \sigma}-\sum_{\mathbf{k}}\left[\Delta c_{\mathbf{k}, \uparrow}^{\dagger} c_{-\mathbf{k}, \downarrow}^{\dagger}+\Delta^{*} c_{-\mathbf{k}^{\prime}, \downarrow} c_{\mathbf{k}^{\prime}, \uparrow}\right]
$$

This Hamiltonian describes what is known as an $s$-wave superconductor since the bound electrons have a singlet symmetry in their spins and is therefore $s$-wave in its orbital wavefunction (figure 1.3). This lets us write that the solution for $\Delta$ can be taken as a constant. In general $\Delta$ can be complex and so we may write it by absorbing its complex nature as a phase factor $\Delta=e^{i \varphi_{s}}|\Delta|$. This Hamiltonian is also quadratic in operators which means that it may be diagonalised and this diagonalisation is known as a Bogoliubov transformation. This allows us to write new operators:

$$
\begin{aligned}
\gamma_{\mathbf{k}, \uparrow} & =u_{\mathbf{k}} c_{\mathbf{k}, \uparrow}-v_{\mathbf{k}} c_{-\mathbf{k}, \downarrow}^{\dagger} \\
\gamma_{-\mathbf{k}, \downarrow} & =v_{\mathbf{k}} c_{\mathbf{k}, \uparrow}^{\dagger}+u_{\mathbf{k}} c_{-\mathbf{k}, \downarrow}
\end{aligned}
$$

where the quantum amplitudes $u_{\mathbf{k}}$ and $v_{\mathbf{k}}$ are related to the probability of pairs being occupied and obey the relation $\left|u_{\mathbf{k}}\right|^{2}+\left|v_{\mathbf{k}}\right|^{2}=1$. The required form of $u_{\mathbf{k}}$ and $v_{\mathbf{k}}$ can be easily worked out and give

$$
\begin{array}{r}
u_{\mathbf{k}}=\sqrt{\frac{1}{2}\left(1+\frac{\varepsilon_{\mathbf{k}}-\mu}{E_{\mathbf{k}}}\right)} \\
v_{\mathbf{k}}=e^{i \varphi_{s}} \sqrt{\frac{1}{2}\left(1-\frac{\varepsilon_{\mathbf{k}}-\mu}{E_{\mathbf{k}}}\right)}
\end{array}
$$

where $E_{\mathbf{k}}=\sqrt{\left(\varepsilon_{\mathbf{k}}-\mu\right)^{2}+|\Delta|^{2}}$ and $\varphi_{s}$ is the phase of $\Delta$, which here turns out to 
also be the phase between $u_{\mathbf{k}}$ and $v_{\mathbf{k}}$. This results in the diagonalised Hamiltonian

$$
H_{B C S}^{M F}=\sum_{\mathbf{k}, \sigma} E_{\mathbf{k}} \gamma_{\mathbf{k}, \sigma}^{\dagger} \gamma_{\mathbf{k}, \sigma}
$$

where we see now that $E_{\mathbf{k}}$ are the excitation energies of the quasi-particles and that the smallest such energy possible is $|\Delta|$. This is where we come to call $\Delta$ the superconducting energy gap. From this the celebrated superconducting ground state is formulated to be

$$
\begin{aligned}
\left|\phi_{B C S}\right\rangle & =\prod_{\mathbf{k}}\left(\gamma_{\mathbf{k}, \uparrow} \gamma_{-\mathbf{k}, \downarrow}\right)\left|\phi_{0}\right\rangle \\
& =\prod_{\mathbf{k}} v_{\mathbf{k}}\left(u_{\mathbf{k}}+v_{\mathbf{k}} c_{\mathbf{k}, \uparrow}^{\dagger} c_{-\mathbf{k}, \downarrow}^{\dagger}\right)\left|\phi_{0}\right\rangle
\end{aligned}
$$

The superconducting gap can also be expressed in terms of the ground state and the definitions of $u_{\mathbf{k}}, v_{\mathbf{k}}$ and $\Delta$. By starting with the definition (1.2) and performing some simple operator algebra one can find:

$$
\begin{array}{r}
\Delta=g \sum_{\mathbf{k}}\left\langle\phi_{B C S}\left|c_{-\mathbf{k}, \downarrow} c_{\mathbf{k}, \uparrow}\right| \phi_{B C S}\right\rangle \\
=g \sum_{\mathbf{k}} u_{\mathbf{k}}^{*} v_{\mathbf{k}}=e^{i \varphi_{s}}|\Delta| \frac{g}{2} \sum_{\mathbf{k}} \frac{1}{\sqrt{\left(\varepsilon_{\mathbf{k}}-\mu\right)^{2}+|\Delta|^{2}}}
\end{array}
$$

This equation is called the self-consistency equation since we find now that $\Delta$ depends on itself. By presuming that $\Delta$ is non-zero one can solve this readily by transforming the sum over momentum into an integral in energy, the result of which gives:

$$
|\Delta|=\frac{\hbar \omega_{D}}{\sinh \left(\frac{1}{g N}\right)}
$$

where $N$ is the density of states and presumed to be constant in the relevant thin shell about the Fermi energy. This is the classic $s$-wave pairing superconducting gap equation at zero temperature and describes well most conventional superconductors.

\subsubsection{Unconventional Superconductivity}

Where for conventional superconductors much of the microscopic theory has already been completed and well understood, unconventional superconductors are wild exotic materials that are, at the moment, not completely understood and whose theoretical natures are not fully established yet.

Before discussing unconventional superconductivity though it will be instructive 
to briefly touch on the symmetries that the superconducting pair potential must have. Since we are considering pairing between electrons, any solution that we have for the system must necessarily be anti-symmetric due to the fermionic nature of electrons. We know that the wavefunction of the pairing can be partitioned into a product of a spin part and a space part. Naturally now either the spin part is odd and the space part is even or the spin is even and space is odd which ensures that, overall, the wavefunction is anti-symmetric. In BCS theory the spin part of the wavefunction is odd, and therefore the space part is even, which is what we call singlet pairing. Generally, to understand the space part of the wavefunction we may expand it in terms of spherical harmonics, i.e. $\phi_{\text {space }}(\mathbf{r}) \propto \sum_{l,|m| \leq l} r^{l} Y_{l}^{m}$. Now since, in BCS, the space part is even then it will only contain terms that are even $(l=0,2, \ldots)$. This means that the leading term in the expansion is a constant, which is what we take in normal BCS theory as in equation (1.10). This is commonly labelled $s$-wave pairing due to the leading term we take in the spherical harmonic expansion as in table 1.1. In contrast to this unconventional superconductivity occupies all other

Table 1.1: The names and expansions of the first four Spherical Harmonics.

\begin{tabular}{|c|c|c|}
\hline $\begin{array}{c}\text { Spherical } \\
\text { Harmonic }\end{array}$ & Name & Expansion \\
\hline$l=0$ & $s$-wave & $\propto r^{0} Y_{0}^{0}(\theta, \phi)$ \\
$l=1$ & $p$-wave & $\propto r^{1} Y_{1}^{m}(\theta, \phi)$ \\
$l=2$ & $d$-wave & $\propto r^{2} Y_{2}^{m}(\theta, \phi)$ \\
$l=3$ & $f$-wave & $\propto r^{3} Y_{3}^{m}(\theta, \phi)$ \\
\hline
\end{tabular}

types of pairing; higher singlet harmonics such as $d$-wave pairing and triplet pairing such as $p$ and $f$-wave pairing where the spin now is even which allows for an odd space part. The spin symmetries of given pairing will determine whether it will have singlet or triplet space symmetries. Figure 1.3 captures this information and gives examples of some of the discovered unconventional superconducting materials that fit the different symmetries.

Unconventional superconductors were first postulated as a response to the discovery of 'high-temperature' superconductors which does not fit well with the predictions of BCS superconductivity. Here 'high-temperature' is generally thought to mean superconductors with a critical temperature of the order of or larger than the boiling point of Nitrogen $(77 \mathrm{~K})$. The first proper group of materials that breached the $77 \mathrm{~K}$ mark were a new class of ceramics called the cuprates as they all have $\mathrm{CuO}$ in their chemical composition. The first of these was $\mathrm{LaBaCuO}_{4}$ which was discovered in 1986 [63] and had a critical temperature of 35.1K. Soon after there was rapid increase in the critical temperatures that could be obtained by the cuprates as can 


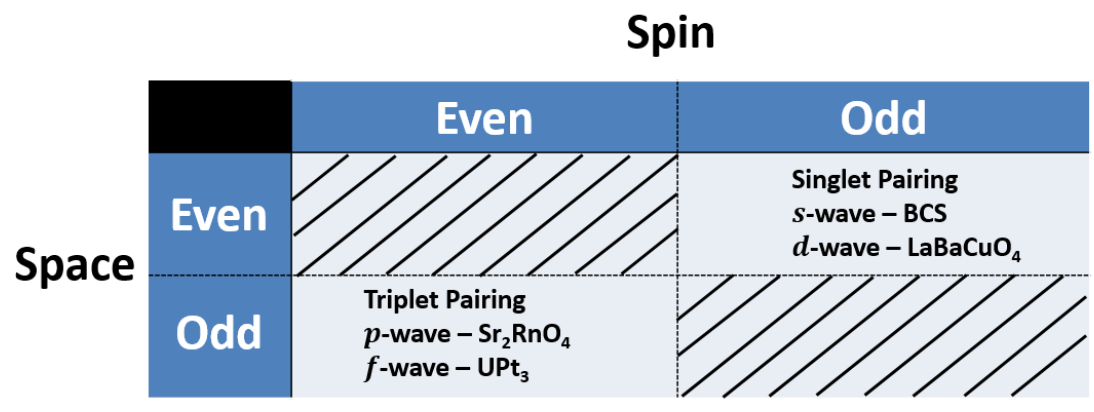

Figure 1.3: A table that shows the allowed products of space and spin parts of the superconducting states wavefunction and the resultant symmetries that exist.

be seen from figure 1.4, including mercury, barium, calcium, copper oxide, which has a critical temperature of $138 \mathrm{~K}$ at ambient temperature [61]. The cuprates and their a-typical structure demanded different theoretical understanding compared to BCS theory. Current understanding places their superconducting pairing as having $d$-wave symmetry which was first proposed by Bickers et al. [65]. This was based upon a description of the material using resonating valence bond theory [66] and has been confirmed to be a direct consequence of such a description [67]. This has also been experimentally confirmed by direct observation of the excitation spectrum as captured by Angle Resolved Photoemission Spectroscopy (ARPES) [68, 69].

Other forms of unconventional superconductivity have been proposed such as $p$ wave superconductivity which are part of the spin-triplet class of superconductors. Spin-triplet pairing involves the superconducting pairing having a symmetrical spin state, i.e. swapping the two spins does not change the sign of the state. This state, like most unconventional superconductors, is also characterised by an anisotropic gap function or order parameter which means that it is not a constant. Triplet pairing, due to its spin symmetry, requires anti-symmetric orbitals (space part) which if we expand in spherical harmonics leads to the allowed odd harmonics such as $p$-wave $(l=1), f$-wave $(l=3)$, etc. Current key materials to have triplet pairing is the heavy fermion material $\mathrm{UPt}_{3}$ with reported $f$-wave pairing [70] and $\mathrm{Sr}_{2} \mathrm{RuO}_{4}$ with reported chiral $p$-wave pairing [71]. However, up till now there has been scant evidence of $p$ wave superconductivity experimentally due its sensitivity to disorder, characterised by several theoretical studies $[56,57]$. The disorder inhibits the superconducting pairing and lowers the critical temperature of such materials, making it hard to identify any superconducting behaviour at all. This is expressed in the universal formula [57] relating the critical temperature $T_{c}$ to the critical temperature in the 


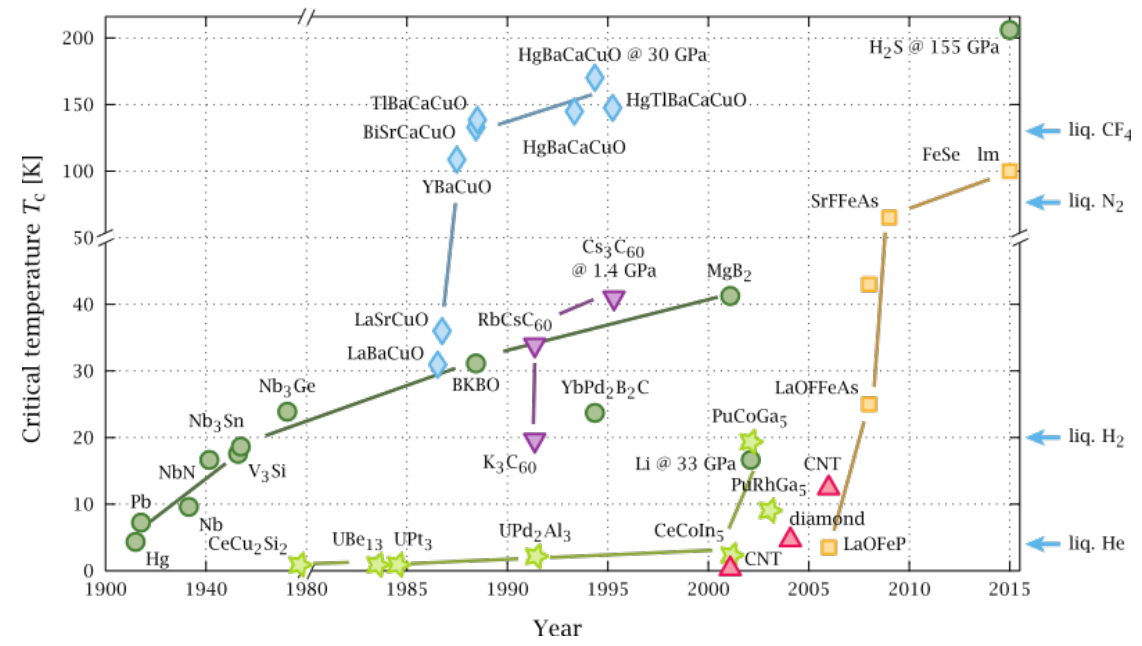

Figure 1.4: Graph showing the critical temperatures of known superconductors from the first discovery in 1911 to 2015. The colours represent different types of superconductors found; green circles represent BCS superconductors, light green stars are heavy fermion based superconductors, blue diamonds are the cuprates, purple inverted triangles are Buckminsterfullerene-based superconductors, red triangles are carbon-allotropes, and orange squares are Iron-pnictogen-based superconductors. Picture taken from [64].

absence of disorder $T_{c 0}$ :

$$
\ln \left(\frac{T_{c 0}}{T_{c}}\right)=\psi\left(\frac{1}{2}+\frac{\hbar}{4 \pi \tau k_{B} T_{c}}\right)-\psi\left(\frac{1}{2}\right)
$$

where $\psi$ denotes the Digamma function, $\tau$ the quasiparticle scattering time, and $k_{B}$ the Boltzmann constant. The increase in impurities will serve to lower $\tau$ and will thus reduce the critical temperature of the materials.

Another form of superconductivity comes from considering multi-band superconductivity. Up until now we have only talked about superconductivity where electron pairing occurs between electrons from the same band. However, one can extend this to systems that have two or more bands and look at either intraband pairing or interband pairing. Intraband pairing would describe pairing between electrons within a single band. This type of pairing describes superconducting states in $\mathrm{MgB}_{2}$ [72, 73] and iron pnictides $[74,75]$. On the other hand interband pairing pairs electrons from differing bands and are expected in a wide variety of topological-type materials such as $\mathrm{Cu}_{x} \mathrm{Bi}_{2} \mathrm{Se}_{3}$ [76-78]. They are expected in these types of materials since band-crossings play essential roles in realizing topologically nontrivial states. These interband couplings can also open the door to both spin-singlet and spin-triplet order parameters as the band index can give the wavefunction its needed asymmetry as required by the fermionic nature of the electrons. This may be the way in which 
we can construct a spin-triplet $s$-wave pairing in $\mathrm{SmN}$. However, it has been reported that such pairing is fragile in the presence of random non-magnetic impurities [79].

In our model of $\mathrm{SmN}$ we are attempting to combine $p$-wave superconductivity in a two-band environment in the hopes that this will explain the existence of seemingly $p$-wave symmetry pairing [5].

\subsection{3 $p$-wave Superconductivity}

Since we are attempting to describe $\mathrm{SmN}$ with a possible $p$-wave superconducting pairing we will describe a simple one-band case as developed thanks to private communications with Moghadden et al. [80].

Considering one spin polarised band we see that the pairing will be a spintriplet: $\Delta_{\uparrow \uparrow}$. This pairing must have an odd orbital form in keeping with fermionic dynamics as the spin component of the wavefunction is naturally symmetric. In this type of superconductivity since both spins are in the same direction we may drop the electron's spin indices. The Hamiltonian of such a system may be written as:

$$
H=\sum_{\mathbf{k}} \xi_{\mathbf{k}} c_{\mathbf{k}}^{\dagger} c_{\mathbf{k}}-\frac{1}{2} \sum_{\mathbf{k}, \mathbf{k}^{\prime}} V_{\mathbf{k}, \mathbf{k}^{\prime}} c_{\mathbf{k}}^{\dagger} c_{-\mathbf{k}}^{\dagger} c_{-\mathbf{k}^{\prime}} c_{\mathbf{k}^{\prime}}
$$

Here $\xi_{\mathbf{k}}$ is the band dispersion about the chemical potential $\mu$ and $V_{\mathbf{k}, \mathbf{k}^{\prime}}$ is the interaction potential which is assumed to be attractive. Here the form of $V_{\mathbf{k}, \mathbf{k}^{\prime}}$ is unknown but the functional form is assumed to be a function of $\left|\mathbf{k}-\mathbf{k}^{\prime}\right|$. From this point on much of the formalism follows closely in comparison to BCS theory, but with some meaningful differences.

The first step is to apply the mean-field approximation to (1.12) by making the definition for $\Delta$ be:

$$
\Delta(\mathbf{k})=\sum_{\mathbf{k}^{\prime}} V_{\mathbf{k}, \mathbf{k}^{\prime}}\left\langle c_{-\mathbf{k}^{\prime}} c_{\mathbf{k}^{\prime}}\right\rangle
$$

Note that in difference to BCS theory that the pairing potential $\Delta_{\mathbf{k}}$ has explicit $\mathbf{k}$ dependence. This allows us to write the mean-field Hamiltonian:

$$
H_{M . F}=\sum_{\mathbf{k}} \xi_{\mathbf{k}} c_{\mathbf{k}}^{\dagger} c_{\mathbf{k}}-\sum_{\mathbf{k}}\left[\Delta_{\mathbf{k}} c_{\mathbf{k}}^{\dagger} c_{-\mathbf{k}}^{\dagger}+\Delta_{\mathbf{k}}^{*} c_{-\mathbf{k}} c_{\mathbf{k}}\right]
$$

Now since (1.14) is quadratic in operators it can be diagonalised in the same way by using the Bogoliubov transformation:

$$
\begin{aligned}
\gamma_{\mathbf{k}} & =u_{\mathbf{k}} c_{\mathbf{k}}-v_{\mathbf{k}} c_{-\mathbf{k}}^{\dagger} \\
\gamma_{-\mathbf{k}} & =v_{\mathbf{k}} c_{\mathbf{k}}^{\dagger}+u_{\mathbf{k}} c_{-\mathbf{k}}
\end{aligned}
$$


where again $u_{\mathbf{k}}$ and $\mathbf{k}$ obey $\left|u_{\mathbf{k}}\right|^{2}+\left|v_{\mathbf{k}}\right|^{2}=1$ and $u_{\mathbf{k}}$ and $\mathbf{k}$ have the form

$$
\begin{array}{r}
u_{\mathrm{k}}=\sqrt{\frac{1}{2}\left(1+\frac{\xi_{\mathrm{k}}}{E_{\mathrm{k}}}\right)} \\
v_{\mathrm{k}}=\frac{\Delta_{\mathrm{k}}}{\left|\Delta_{\mathrm{k}}\right|} \sqrt{\frac{1}{2}\left(1-\frac{\xi_{\mathrm{k}}}{E_{\mathrm{k}}}\right)}
\end{array}
$$

where $E_{\mathbf{k}}=\sqrt{\xi_{\mathbf{k}}^{2}+\left|\Delta_{\mathbf{k}}\right|^{2}}$. Note that at this point there is no difference between this and the BCS theory as the prefactor $\frac{\Delta_{\mathbf{k}}}{\left|\Delta_{\mathbf{k}}\right|}$ is just another way of writing the phase between $u_{\mathbf{k}}$ and $v_{\mathbf{k}}$. These choices of quantum amplitudes allows us to once again write our Hamiltonian as $H_{M . F}=\sum_{\mathbf{k}} E_{\mathbf{k}} \gamma_{\mathbf{k}}^{\dagger} \gamma_{\mathbf{k}}$ and our expected form for the ground state $\left|\phi_{G}\right\rangle=\prod_{\mathbf{k}} \gamma_{\mathbf{k}}\left|\phi_{0}\right\rangle$. Here we see that the only differences between BCS and this spinless $p$-wave formalism is that the spin of the electrons are not important and that the pair potential cannot only be a constant and must depend on $\mathbf{k}$ in a non-trivial way.

At zero temperature one can construct the self-consistency equation which can be easily worked out to be

$$
\begin{aligned}
\Delta_{\mathbf{k}} & =\sum_{\mathbf{k}^{\prime}} V_{\mathbf{k}, \mathbf{k}^{\prime}}\left\langle c_{-\mathbf{k}^{\prime}} c_{\mathbf{k}^{\prime}}\right\rangle=\sum_{\mathbf{k}^{\prime}} V_{\mathbf{k}, \mathbf{k}^{\prime}} u_{\mathbf{k}^{\prime}}^{*} v_{\mathbf{k}^{\prime}} \\
& =\frac{1}{2} \sum_{\mathbf{k}^{\prime}} V_{\mathbf{k}, \mathbf{k}^{\prime}} \frac{\Delta_{\mathbf{k}}}{\sqrt{\xi_{\mathbf{k}}^{2}+\left|\Delta_{\mathbf{k}}\right|^{2}}}
\end{aligned}
$$

To solve this self-consistently there are three possible pairing symmetries that $\Delta_{\mathbf{k}}$ may take. These are governed by the three spherical harmonics $Y_{l}^{m}(l=1, m=$ $-1,0,1)[81]$ in the following way:

$$
\Delta(\mathbf{k})^{(m)} \equiv \Delta_{0}^{(m)} \frac{k}{k_{F}} Y_{1}^{m}(\theta, \varphi)
$$

where $m=0, \pm 1$ corresponds to what are called the $p_{z^{-}}$and $\left(p_{x} \pm i p_{y}\right)$-pairing symmetries. In addition to this since $V_{\mathbf{k}, \mathbf{k}^{\prime}}$ is assumed to be a function of $\left|\mathbf{k}-\mathbf{k}^{\prime}\right|$ then it may be expanded in powers of $\mathbf{k} \cdot \mathbf{k}^{\prime}$. Now since the pairing potential is $p$-wave and is thus defined by its expansion in spherical harmonics $r^{1} Y_{1}^{m}(\theta, \varphi)$ as in table 1.1 and in equation (1.18). Terms entering into the gap equation must therefore be of the same symmetry. We must conclude that the only surviving term of the $V_{\mathbf{k}, \mathbf{k}^{\prime}}$ expansion will be the term linear in $\mathbf{k} \cdot \mathbf{k}^{\prime}: V_{2} \mathbf{k} \cdot \mathbf{k}^{\prime}$. This is due to this linear term being the only such term to contain the required form in the radial direction, i.e. $r^{1}$ or in this case $\mathbf{k}^{1}$. Using this result and converting the sum in (1.17) to an integral, 
where $\Omega$ is the volume, via

$$
\sum_{\mathbf{k}^{\prime}} \rightarrow \frac{\Omega}{(2 \pi)^{3}} \int d k^{\prime 3}=\frac{\Omega}{(2 \pi)^{3}} \int d k^{\prime} k^{\prime 2} \int_{0}^{\pi} d \theta^{\prime} \sin \theta^{\prime} \int_{0}^{2 \pi} d \varphi^{\prime}
$$

a self-consistent solution can be found for (1.17) for all three $p$-wave symmetries. The results of such an integration give the results:

$$
\begin{aligned}
\Delta_{0}^{(0)} & =\omega_{D} 2 e^{\frac{1}{3}} \exp \left(-\frac{3}{\lambda N\left(\varepsilon_{F}\right)}\right) \\
\Delta_{0}^{( \pm 1)} & =\omega_{D} e^{\frac{5}{6}} \exp \left(-\frac{3}{\lambda N\left(\varepsilon_{F}\right)}\right)
\end{aligned}
$$

where $\lambda=\Omega V_{2} k_{F}^{2}$ and $N\left(\varepsilon_{F}\right)=\frac{k_{F}^{2}}{2 v_{F} \pi^{2}}$. We will be adapting this formalism in our description of SmN's $p$-wave superconductivity.

\section{$1.3 \quad$ The $\mathrm{k} \cdot \mathrm{P}$ Method}

In order to model our REN of choice $\mathrm{SmN}$, we will be utilising the results of the $\mathbf{k} \cdot \mathbf{p}$ method $[82,83]$, the basics of which are reviewed below. As in [82] we begin with a Hamiltonian containing only a kinetic operator, a periodic potential, and a spin-orbit coupling term.

$$
H=\frac{p^{2}}{2 m_{0}}+V(\mathbf{r})+\alpha_{0}(\boldsymbol{\sigma} \times \nabla V) \cdot \mathbf{p}
$$

with $\mathbf{p}=-i \hbar \nabla, \alpha_{0}=\frac{\hbar}{4 m_{0}^{2} c^{2}}$ and bold symbols represent vector quantities. From considering the Schrödinger equation for one electron

$$
H \psi_{n \mathbf{k}}(\mathbf{r})=E_{n}(\mathbf{k}) \psi_{n \mathbf{k}}(\mathbf{r})
$$

we presume that solutions may take the form of Bloch states

$$
\psi_{n \mathbf{k}}(\mathbf{r})=e^{i \mathbf{k} \cdot \mathbf{r}} u_{n \mathbf{k}}(\mathbf{r})
$$

where the $u_{n \mathbf{k}}(\mathbf{r})$ corresponding to different bands $n$ are orthogonal to each other, i.e.

$$
\left\langle u_{n \mathbf{k}} \mid u_{n^{\prime} \mathbf{k}}\right\rangle=\int_{V} d V u_{n \mathbf{k}}^{*} u_{n^{\prime} \mathbf{k}}=\delta_{n n^{\prime}} \frac{V}{(2 \pi)^{3}}
$$

with $V$ the volume of the unit cell.

Now we may substitute the solution (1.23) into (1.22) to find an eigenvalue 
equation for $u_{n, \mathbf{k}}$. Initially we apply the operator $\mathbf{p}$ and $p^{2}$ to $\psi_{n \mathbf{k}}$ to find

$$
\begin{gathered}
\mathbf{p}\left(\psi_{n \mathbf{k}}\right)=-i \hbar \boldsymbol{\nabla}\left(e^{i \mathbf{k} \cdot \mathbf{r}} u_{n \mathbf{k}}\right)=\hbar \mathbf{k} e^{i \mathbf{k} \cdot \mathbf{r}} u_{n \mathbf{k}}+e^{i \mathbf{k} \cdot \mathbf{r}} \mathbf{p} u_{n \mathbf{k}} \\
p^{2}\left(\psi_{n \mathbf{k}}\right)=-i \hbar \boldsymbol{\nabla}\left(\hbar \mathbf{k} e^{i \mathbf{k} \cdot \mathbf{r}} u_{n \mathbf{k}}+e^{i \mathbf{k} \cdot \mathbf{r}} \mathbf{p} u_{n \mathbf{k}}\right)=e^{i \mathbf{k} \cdot \mathbf{r}}\left(\hbar^{2} k^{2}+2 \hbar \mathbf{k} \cdot \mathbf{p}+p^{2}\right) u_{n \mathbf{k}}
\end{gathered}
$$

Thus (1.22) becomes

$$
\begin{gathered}
e^{i \mathbf{k} \cdot \mathbf{r}}\left[\frac{\hbar^{2} k^{2}}{2 m_{0}}+\frac{\hbar \mathbf{k} \cdot \mathbf{p}}{m_{0}}+\frac{p^{2}}{2 m_{0}}+V(\mathbf{r})\right] u_{n \mathbf{k}}+e^{i \mathbf{k} \cdot \mathbf{r}} \alpha_{0}(\boldsymbol{\sigma} \times \boldsymbol{\nabla} V) \cdot(\hbar \mathbf{k}+\mathbf{p}) u_{n \mathbf{k}}=E_{n}(\mathbf{k}) e^{i \mathbf{k} \cdot \mathbf{r}} u_{n \mathbf{k}} \\
\underbrace{\left[\frac{p^{2}}{2 m_{0}}+V(\mathbf{r})+\alpha_{0}(\boldsymbol{\sigma} \times \nabla V) \cdot \mathbf{p}\right]}_{H_{0}} u_{n \mathbf{k}}+\underbrace{\frac{\hbar}{m_{0}}\left(\mathbf{k} \cdot \boldsymbol{\pi}+\frac{\hbar}{2} k^{2}\right)}_{H_{\mathbf{k} \cdot \mathbf{p}}} u_{n \mathbf{k}}=E_{n}(\mathbf{k}) u_{n \mathbf{k}}(1.27)
\end{gathered}
$$

with $\boldsymbol{\pi}=\mathbf{p}+\alpha_{0} m_{0}(\boldsymbol{\sigma} \times \boldsymbol{\nabla} V)$ and we may write $H(\mathbf{k})=H_{0}+H_{\mathbf{k} \cdot \mathbf{p}}$. Equation (1.27) is the $\mathbf{k} \cdot \mathbf{p}$ equation and is the starting point for our treatment of $\mathrm{SmN}$. If the set of $u_{n \mathbf{k}}$ used form a complete set then one may find the dispersion relation throughout the entire Brillouin zone by diagonalising $\left\langle u_{n \mathbf{k}}|H(\mathbf{k})| u_{n^{\prime} \mathbf{k}}\right\rangle$. This is the power of the $\mathbf{k} \cdot \mathbf{p}$ method. As well as this if we only consider one single $u_{n \mathbf{k}}$ function then we may apply non-degenerate perturbation theory to find its energy dispersion. Recalling perturbation theory for a system of the form $H=H_{0}+\lambda V$ with $\lambda$ small then the energy dispersion to second order can be given by

$$
E_{n}=E_{n}^{(0)}+\lambda\left\langle n^{(0)}|V| n^{(0)}\right\rangle+\lambda^{2} \sum_{k \neq n} \frac{\left|\left\langle n^{(0)}|V| k^{(0)}\right\rangle\right|^{2}}{E_{n}^{(0)}-E_{k}^{(0)}}
$$

\subsection{Outline}

The goal of this thesis is to investigate the nature of superconductivity in $\mathrm{SmN}$, a ferromagnetic semi-conductor, to shed light on recent experimental results that indicate the onset of superconductivity in this material. To understand the superconductivity of $\mathrm{SmN}$ we will attempt to find some pair potential $\Delta$ in a system based on SmN's bandstructure in a similar way as seen in subsection 1.2.1 and subsection 1.2.3. We will also work to see what happens to the pairing if we consider the case when a band crossing occurs near the Fermi energy. The model Hamiltonians that we will be working with will all be treated with the mean-field approximation and assuming that the potential pairing together electrons comes from phonons in the long wavelength limit. Focus will be given to finding the pairing potential $\Delta$ which contains key information about superconductivity in a given system and attempts will be made to find it in a closed form (i.e. an analytic expression for $\Delta$ ). 
In chapter 2 we develop the effective model that we will use to probe the superconductivity of SmN. In this chapter we will consider the bandstructure of SmN, where recent experiments have placed the $4 f$ energy band close to the bottom of the conduction band. We assume that the electron-phonon interaction is the mechanism through which we expect the electrons to pair to one-another. As such, we form a model for this interaction in the long wavelength model which we may input into our model Hamiltonian. This is done by considering how the energy bands will change due to a phonon travelling through the crystal, which allows us to predict how strong the interaction will be. Next, in chapter 3 we will investigate $p$-wave pairing present between electrons in SmN's $5 d$-band. We apply the mean-field approximation to our model Hamiltonian and diagonalise this system in order to find an expression for the pair potential. This is done by constructing a self-consistency equation which we attempt to solve. This has partial success in generating analytic solutions but we are able to find the functional dependence on the effective coupling strength for the resultant pairing. After this, in chapter 4, we instead consider the electron-phonon interaction interacting with energy bands that have hybridised together. We did this to search for more robust pairing since we expect the $p$-wave pairing found in the previous chapter to be sensitive to disorder. This hybridisation has the effect of 'mixing' the pair potential that we found previously from which we will find new pairing between electrons in both of the hybridised bands. Finally, we conclude the thesis in chapter 5 by summarising the results in the previous chapters and providing avenues for potential continuation of this research in the future. 


\section{Chapter 2}

\section{The Model}

\subsection{Band-Structure of SmN}

The bandstructure of a material is a plot of the energy dispersions of the material in reciprocal space, or $\mathbf{k}$ space. A simple example is give in figure 2.1 where the energy bands of the semi-conductor Silicon ( $\mathrm{Si}$ ) are shown as a function of electron wavevector $\mathbf{k}$. We see that as with all semi-conductors there is a band gap; a range of energies where no states are available for occupation. The conduction bands are those bands above this band gap while the valence bands are those below. Si is in fact an example of a semi-conductor with an indirect band gap since the top of the valence band (at $\Gamma$ ) is not in line with the bottom of the conduction band (close to $X)$. This is the same type of semi-conductor as $\mathrm{SmN}$, although $\mathrm{SmN}$ has a more complicated structure overall. The $y$-axis moves up in energy and the $x$-axis along $\mathbf{k}$ space, which does not move in a straight line but rather moves from predetermined points of high symmetry such as the $\Gamma$ point or the $X$ point. These points will depend on the shape of the crystal structure of the material. We are studying $\mathrm{SmN}$ which has a cubic crystal structure, therefore its reciprocal space has a body centered cubic (BCC) structure. Figure 2.2 shows the high symmetry points for such a crystal and how they connect to each other. At zero temperature the Fermi level will sit directly in the middle of the band gap, although this can be moved up and down by doping the semiconductor with either extra electrons or holes in the form of foreign atoms. In SmN's case this can also be achieved by introducing Nitrogen vacancies.

For most materials, bandstructure calculations are a straightforward task that yield widely accepted results. The calculated bandstructures are usually probed by conducting experiments on the material and finding its density of states (DOS) among other techniques. However, there are materials where bandstructure calculations are not so easy to compute. The RENs are one such material class that 


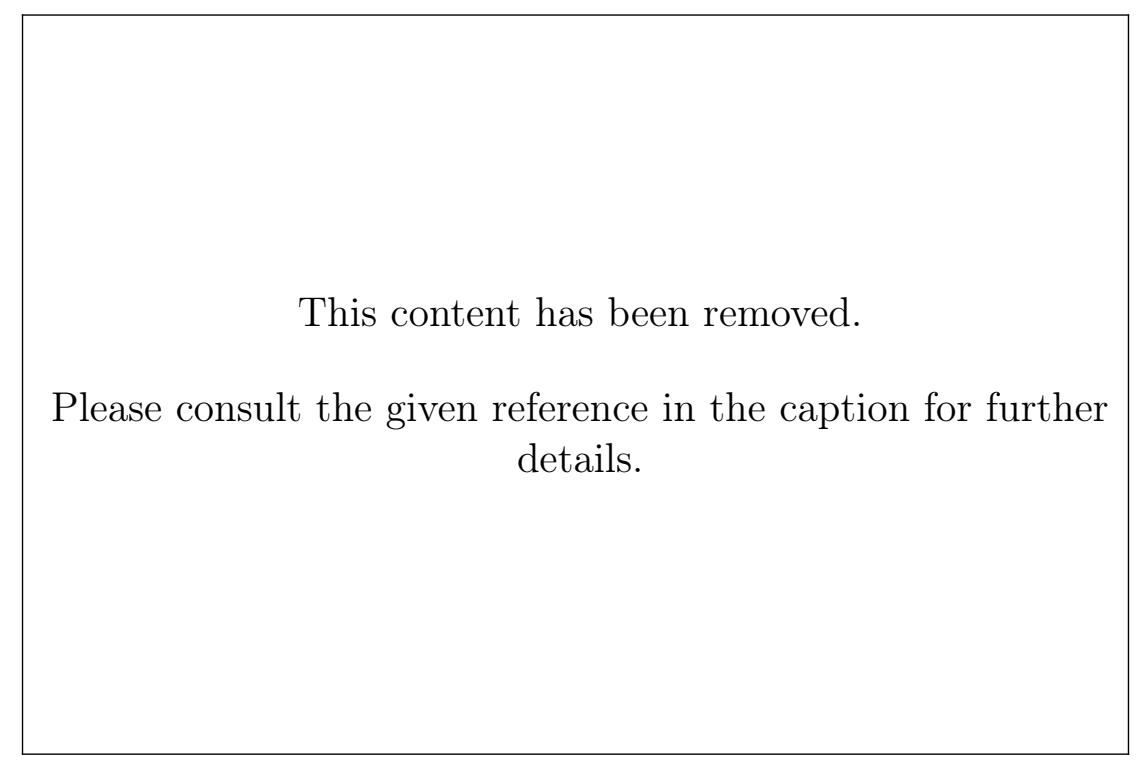

Figure 2.1: The bandstructure of Silicon. There is a band-gap between the top of the valence band at the $\Gamma$ point and the bottom of the conduction band around the $X$ point. The positions of these points show that it is an indirect band-gap semiconductor since electrons with just enough energy to be excited into the conduction band must also be shifted in momentum (k) space by an incident phonon. Figure taken from [84] (see section 2.1.5 under the Semiconductor Physics chapter).

have bandstructures that are complicated to model due, almost exclusively, to the highly localised and correlated $4 f$ electrons. There has been difficulty isolating the proper bandstructure for SmN due to sparse experimental data and the difficulties of properly modelling the effects of the atomic-like $4 f$ electrons. An example of SmN's bandstructure is seen in figure 2.3 which has been calculated by Larson and Lambrecht et al. [22]. The figure shows the highly atomic $4 f$ bands as the flat bands down at $-5 \mathrm{eVs}$ and below and up at $5 \mathrm{eVs}$ and above, with the one unoccupied majority-spin $4 f$ band hybridising with the conduction band near the Fermi level. It also shows an optical band gap between the conduction band and the valence band at the $X$ point.

There are multiple similarities between the different bandstructures that researchers have calculated such as the five occupied $4 f$ bands far below the Fermi energy and the top eight unoccupied $4 f$ bands far above the Fermi energy and the observed optical band-gap at the $X$ point. However, what has been debated is where to pin the unoccupied majority $4 f$ band that is closest to the Fermi level and how to model the magnitude of the indirect band gap we expect it to have due to its semi-conductor behaviour. Some calculations do not take the localised nature of the $4 f$ bands into account very well which push it far above the Fermi energy [86, 87] while some calculations and experiments place the $4 f$ band near the conduction 


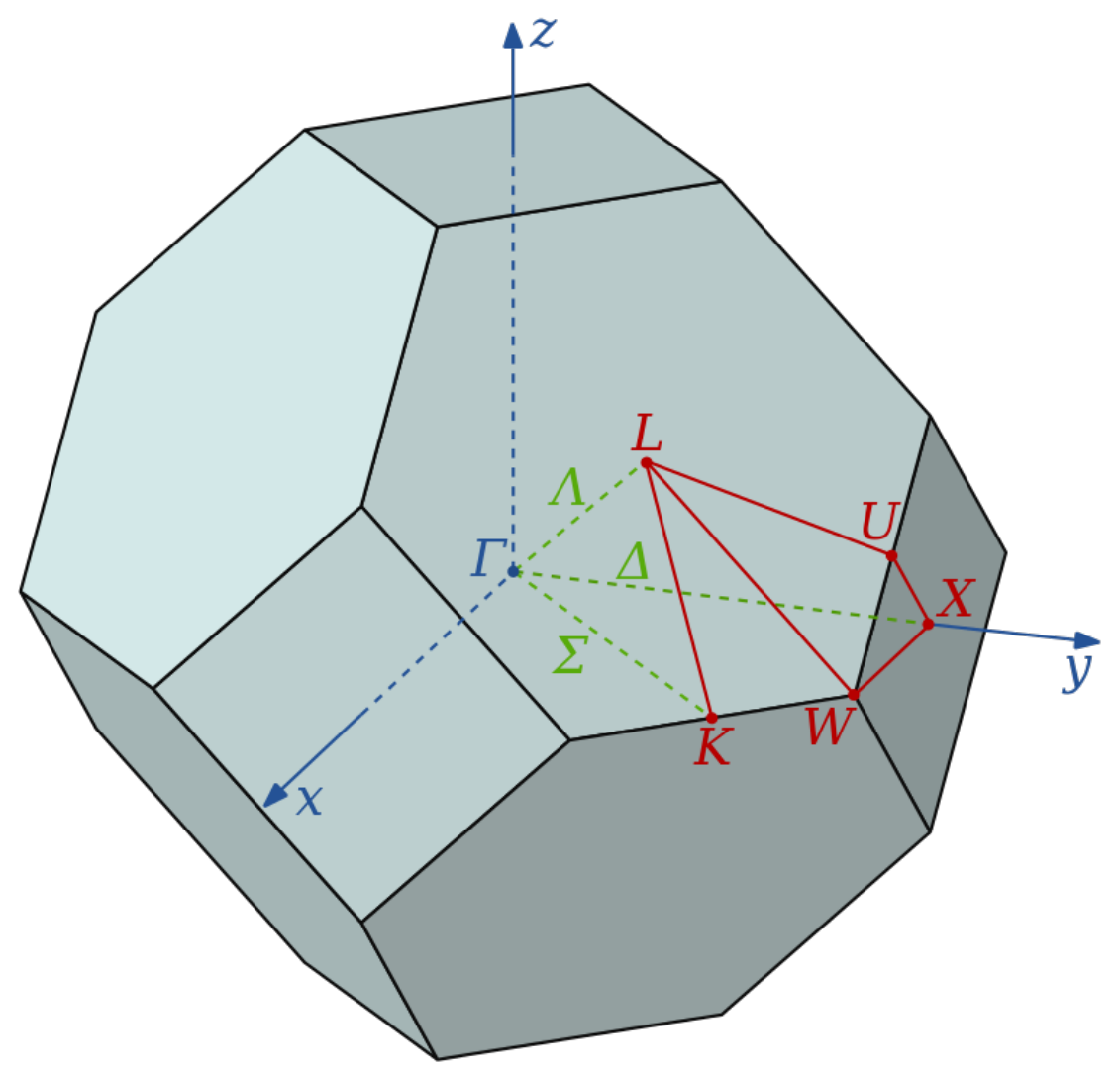

Figure 2.2: The high symmetry points in reciprocal space of SmN. The shaded polygon is the shape of the first Brillouin Zone of SmN. Figure taken from [85].

band minimum $[22,88]$. However, we are fortunate to have recent experimental studies that have probed for the exact location of this closest $4 f$ band $[8,22,89]$.

Informed by these experimental results and the general form of the bandstructure calculations we suppose that the shape of the bandstructure for bands close to the Fermi energy is as shown schematically in figure 2.4. The Sm $4 f$ band is assumed to be flat and dispersion-less for the most part and meets the Sm $5 d$ band near the $X$ point. This forms the bottom of the conduction band and will be the focus of the model. As discussed before there is significant exchange splitting within the bands as well. Since there is large exchange splitting of the bands we may treat each band as spin-polarized i.e. containing electrons of one spin. This also naturally leads to the breaking of time reversal symmetry in the crystal. There is only one spin majority $\mathrm{Sm} 4 f$ band that is located near the Fermi level as in figure 2.4 and the other unoccupied bands are calculated to be at energies $\geq 5 \mathrm{eV}[22$, 87] allowing us to ignore these bands in our low energy model. The location of the Fermi level $E_{F}$ is not well known as well since the size of the indirect band gap between the valence band maximum (VBM) (attributed to the Nitrogen's $2 p$ 


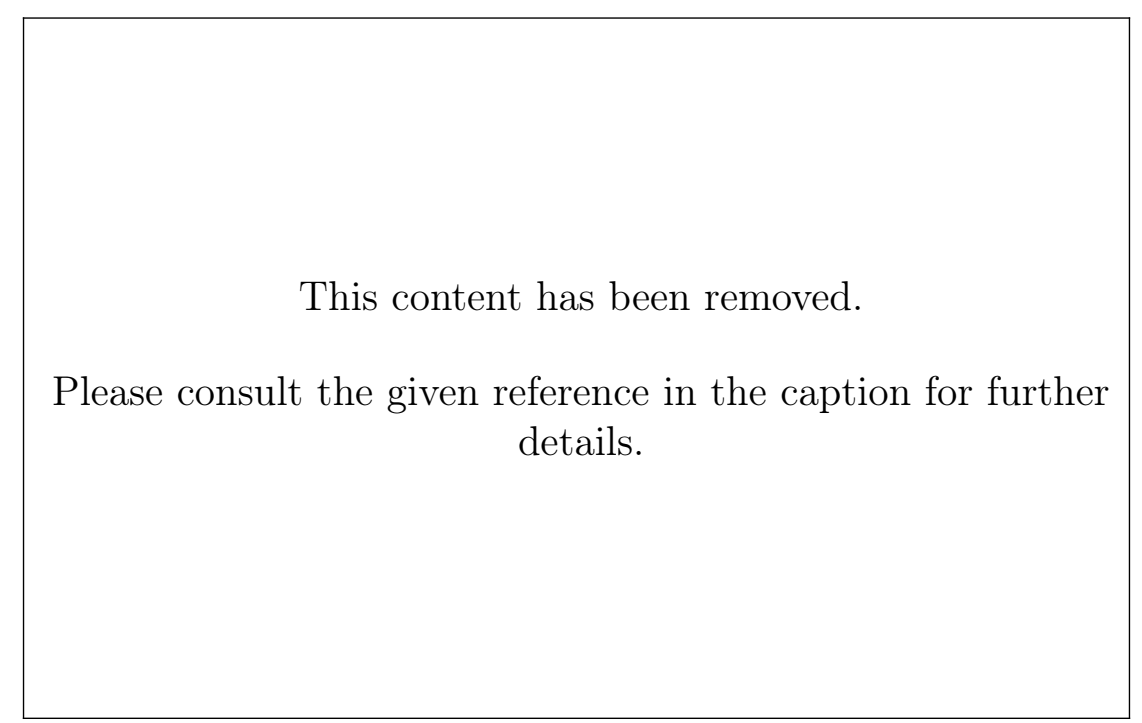

Figure 2.3: Calculated bandstructure of SmN using the LSDA $+\mathrm{U}$ approach [22]. The occupied $4 f$ bands are all located well below the Fermi level at $-5 \mathrm{eV}$. There is a band crossing between the lowest unoccupied $4 f$ level and the Sm $5 d$ level at the $X$ point. There is also significant spin splitting in the band levels as indicated by the dotted line. In this bandstructure calculation there does not seem to be an indirect band-gap between the majority spin bands. Figure taken from [22] (Figure 8).

electrons) at the $\Gamma$ point and the conduction band minimum (CBM) at the $X$ point is not well known. Band calculations have reported $[22,90]$ that there may in fact be no indirect band-gap, however, this is not consistent with experiment [4-9] which reports semiconductor behaviour at temperatures below the ferromagnetic $T_{C}$. Thus we will assume that there is a definite indirect band gap for the majority spin bands which lie lower (higher) in energy for the conduction (valence) band. From this we may then disregard the N $2 p$ bands in our model since these bands should play no role in the transport properties of the conduction band due to the band gap.

\subsection{The Effective Model of SmN}

Moving from the bandstructure of $\mathrm{SmN}$ as developed in the preceding section we may now create an effective model from the bands that are close to the Fermi level. For our model we will only consider the spin minority and majority Sm $5 d$ and spin majority $\operatorname{Sm} 4 f$ bands at the $X$ point to be relevant for transport properties and for superconductivity. As such we expect to be able to write an effective Hamiltonian 


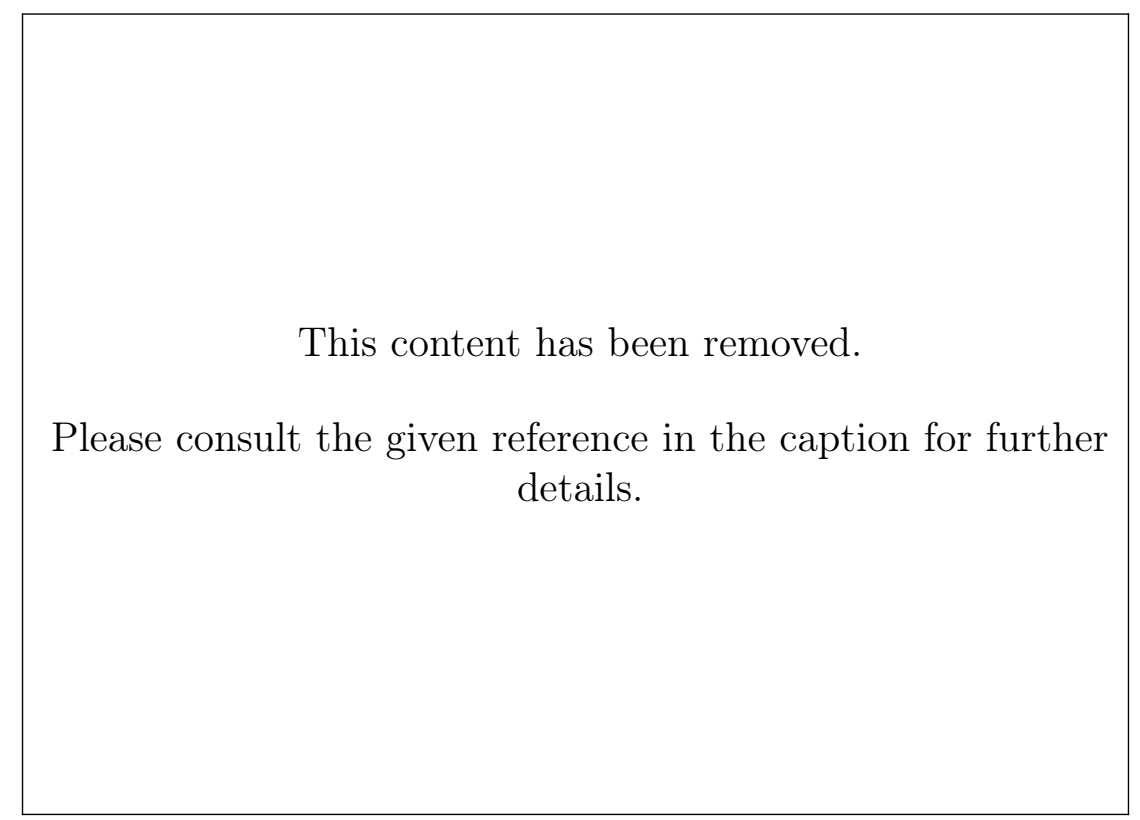

Figure 2.4: Schematic representation of the bandstructure for SmN. The Sm $4 f$ band is presumed to be flat and meets the parabolic Sm $5 d$ band at the $X$ point (hysteresis not shown). There is significant spin splitting within the bands as seen by the difference between the majority spin bands (solid black) and the minority spin bands (dashed red). Figure taken from [8] (Figure 1).

for this system of the form

$$
H_{X}=\left[\begin{array}{ccc}
E_{d \downarrow}(\overline{\mathbf{k}}) & \mathcal{S} . \mathcal{O} . & \mathcal{S} . \mathcal{O} . \\
\mathcal{S} . \mathcal{O} . & E_{d \uparrow}(\overline{\mathbf{k}}) & A \overline{\mathbf{k}} \\
\mathcal{S} . \mathcal{O} . & A \overline{\mathbf{k}} & E_{f b}(\overline{\mathbf{k}})
\end{array}\right]
$$

where $\overline{\mathbf{k}}$ is $\mathbf{k}$ measured from the $X$ point, $E_{d \downarrow,(\uparrow)}(\overline{\mathbf{k}})$ is the energy dispersion of the $5 d$ minority (majority) spin bands, $E_{f b}(\overline{\mathbf{k}})$ is the energy dispersion of the $4 f$ majority spin band, $\mathcal{S} . \mathcal{O}$. is the spin-orbit coupling between the $5 d$ majority and minority spin bands and between the $4 f$ and minority $5 d$ bands, and $A \overline{\mathbf{k}}$ is an interaction term between the majority spin $4 f$ and $5 d$ states found via the $\mathbf{k} \cdot \mathbf{p}$ method. We also presume that at this stage the $\mathcal{S}$.O . terms will be negligibly small due to the large spin-splitting between bands in $\mathrm{SmN}$. To find the elements $A$ we first apply the $\mathbf{k} \cdot \mathbf{p}$ method at the $X$ point.

We begin by calling the $\mathbf{k}$ vector at $X \mathbf{k}_{X}$ and define $\overline{\mathbf{k}}$ to be the $\mathbf{k}$ vector around $\mathbf{k}_{X}$ i.e. $\overline{\mathbf{k}}=\mathbf{k}-\mathbf{k}_{X}$. When $\mathbf{k}=\mathbf{k}_{X}$ equation (1.27) is now

$$
H_{0} u_{n \mathbf{k}_{X}}+\frac{\hbar}{m_{0}}\left(\mathbf{k}_{X} \cdot \boldsymbol{\pi}+\frac{\hbar}{2} k_{X}^{2}\right) u_{n \mathbf{k}_{X}}=E_{n}\left(\mathbf{k}_{X}\right) u_{n \mathbf{k}_{X}}
$$


and we presume now that we are able to solve for and find $E_{n}\left(\mathbf{k}_{X}\right)$. As in [91] we can write

$$
H_{\mathbf{k}_{X}}=H_{0}+\frac{\hbar}{m_{0}}\left(\mathbf{k}_{X} \cdot \boldsymbol{\pi}+\frac{\hbar}{2} k_{X}^{2}\right)
$$

which will act as our unperturbed Hamiltonian at $\mathbf{k}_{X}$. Now we can re-express $H(\mathbf{k})$ as

$$
\begin{aligned}
H(\mathbf{k}) & =H_{0}+H_{\mathbf{k} \cdot \mathbf{p}} \\
& =H_{0}+\frac{\hbar}{m_{0}}\left(\mathbf{k} \cdot \boldsymbol{\pi}+\frac{\hbar}{2} k^{2}\right)+\frac{\hbar}{m_{0}}\left(\mathbf{k}_{X} \cdot \boldsymbol{\pi}+\frac{\hbar}{2} k_{X}^{2}\right)-\frac{\hbar}{m_{0}}\left(\mathbf{k}_{X} \cdot \boldsymbol{\pi}+\frac{\hbar}{2} k_{X}^{2}\right) \\
& =H_{\mathbf{k}_{X}}+\underbrace{\frac{\hbar}{m_{0}}\left(\left(\mathbf{k}-\mathbf{k}_{X}\right) \cdot \boldsymbol{\pi}+\frac{\hbar}{2}\left(k^{2}-k_{X}^{2}\right)\right)}_{H_{p}}
\end{aligned}
$$

and so we may now treat $H_{p}$ as our perturbation to $H_{\mathbf{k}_{X}}$. Since we would like to write everything in terms of $\overline{\mathbf{k}}$ let us rearrange $H_{p}$ to a suitable form first.

$$
\begin{aligned}
& H_{p}=\frac{\hbar}{m_{0}}\left(\mathbf{k}-\mathbf{k}_{X}\right) \cdot \boldsymbol{\pi}+\frac{\hbar^{2}}{2 m_{0}}\left(k^{2}-k_{X}^{2}\right)+\frac{\hbar}{m_{0}}\left(\mathbf{k}-\mathbf{k}_{X}\right) \cdot \hbar \mathbf{k}_{X}-\frac{\hbar}{m_{0}}\left(\mathbf{k}-\mathbf{k}_{X}\right) \cdot \hbar \mathbf{k}_{X} \\
& =\frac{\hbar}{m_{0}}\left(\mathbf{k}-\mathbf{k}_{X}\right) \cdot\left(\boldsymbol{\pi}+\hbar \mathbf{k}_{X}\right)+\frac{\hbar^{2}}{2 m_{0}} \overbrace{\left(k^{2}+k_{X}^{2}-2 \mathbf{k} \cdot \mathbf{k}_{X}\right)}^{\left|\mathbf{k}-\mathbf{k}_{X}\right|^{2}} \\
& =\frac{\hbar}{m_{0}}\left(\overline{\mathbf{k}} \cdot\left(\boldsymbol{\pi}+\hbar \mathbf{k}_{X}\right)+\frac{\hbar}{2} \bar{k}^{2}\right)
\end{aligned}
$$

Now we may perform perturbation theory about the $X$ point by presuming we know the solution set $u_{n \mathbf{k}_{X}}$. In this basis we now find for the $5 d$ majority spin band

$$
\begin{aligned}
E_{d}(\overline{\mathbf{k}}) & =E_{d}(\overline{\mathbf{k}}=0)+\left\langle d\left|H_{p}\right| d\right\rangle+\sum_{n^{\prime} \neq d} \frac{\left|\left\langle d\left|H_{p}\right| n^{\prime}\right\rangle\right|^{2}}{E_{d}(\overline{\mathbf{k}}=0)-E_{n^{\prime}}(\overline{\mathbf{k}}=0)} \\
& =E_{d}(0)+\frac{\hbar}{m_{0}}\left\langle d\left|\overline{\mathbf{k}} \cdot\left(\boldsymbol{\pi}+\hbar \mathbf{k}_{X}\right)+\frac{\hbar}{2} \bar{k}^{2}\right| d\right\rangle+\frac{\hbar^{2}}{m_{0}^{2}} \sum_{n^{\prime} \neq d} \frac{\left|\left\langle d\left|\overline{\mathbf{k}} \cdot\left(\boldsymbol{\pi}+\hbar \mathbf{k}_{X}\right)+\frac{\hbar}{2} \bar{k}^{2}\right| n^{\prime}\right\rangle\right|^{2}}{E_{d}(0)-E_{n^{\prime}}(0)} \\
& =E_{d}(0)+\frac{\hbar^{2}}{2 m_{0}} \bar{k}^{2}+\frac{\hbar}{m_{0}} \overline{\mathbf{k}} \cdot\left(\hbar \mathbf{k}_{X}+\langle d|\boldsymbol{\pi}| d\rangle\right)+\frac{\hbar^{2}}{m_{0}^{2}} \sum_{n^{\prime} \neq d} \frac{\left|\overline{\mathbf{k}} \cdot\left\langle d|\boldsymbol{\pi}| n^{\prime}\right\rangle\right|^{2}}{E_{d}(0)-E_{n^{\prime}}(0)}
\end{aligned}
$$

where $|n\rangle=u_{n \mathbf{k}_{X}}$ and $\left\langle n|W| n^{\prime}\right\rangle=\frac{(2 \pi)^{3}}{V} \int_{V} d V u_{n \mathbf{k}_{X}}^{*} W u_{n^{\prime} \mathbf{k}_{X}}$ for convenience. Considering that the $5 d$ band is at an extremum at $\mathbf{k}_{X}$ then we also expect that any linear terms in the dispersion will go to zero, i.e. $\frac{\partial E_{n}\left(\mathbf{k}_{X}\right)}{\partial k_{i}}=0$. Also by only considering the lower $2 \times 2$ block of (2.1) the diagonal element $E_{d \uparrow}$ will not have second order 
corrections from the $4 f$ band since these will go on the off-diagonal for the $A \overline{\mathbf{k}}$ term. For now we consider only an isotropic case. Thus we may write

$$
\begin{aligned}
E_{d \uparrow}(\overline{\mathbf{k}}) & =E_{d \uparrow}(0)+\frac{\hbar^{2}}{2 m_{0}} \bar{k}^{2}+\frac{\hbar^{2}}{m_{0}^{2}} \bar{k}^{2} \sum_{n^{\prime} \neq d \uparrow, f} \frac{\left|\left\langle d \uparrow|\boldsymbol{\pi}| n^{\prime}\right\rangle\right|^{2}}{E_{d}(0)-E_{n^{\prime}}(0)} \\
& =E_{d \uparrow}(0)+\frac{\hbar^{2}}{2 m_{d \uparrow}} \bar{k}^{2}
\end{aligned}
$$

with $\frac{1}{m_{d \uparrow}}=\frac{1}{m_{0}}+\frac{2}{m_{0}^{2}} \sum_{n^{\prime} \neq d \uparrow, f} \frac{\left|\left\langle d \uparrow|\pi| n^{\prime}\right\rangle\right|^{2}}{E_{d}(0)-E_{n^{\prime}}(0)}$. Naturally based off of the bandstructure diagram we are working with $E_{d \downarrow}$ will have the same from as $E_{d \uparrow}$ but with an additional $E_{e s}$ constant representing the exchange splitting of the bands. This term can be absorbed into a $E_{d \downarrow}(0)$ term, i.e. $E_{d \downarrow}(0)=E_{e s}+E_{d \uparrow}(0)$. Similarly for $E_{f b}$, recognizing that the band is assumed to be flat, we arrive at

$$
E_{f b}(\overline{\mathbf{k}})=E_{f b}(0)+\frac{\hbar^{2}}{2 m_{f b}} \bar{k}^{2}
$$

with $\frac{1}{m_{f b}}=\frac{2}{m_{0}^{2}} \sum_{n^{\prime} \neq d \uparrow, f} \frac{\left|\left\langle f|\boldsymbol{\pi}| n^{\prime}\right\rangle\right|^{2}}{E_{f b}(0)-E_{n^{\prime}}(0)}$ and $m_{f b}$ assumed to be very large. Now we may write our off-diagonal $A \overline{\mathbf{k}}$ terms as $\langle d|H(\mathbf{k})| f\rangle$ and $\langle d|H(\mathbf{k})| f\rangle$ which gives

$$
\begin{aligned}
\langle d \uparrow|H(\mathbf{k})| f\rangle & =\left\langle d \uparrow\left|H_{\mathbf{k}_{X}}+H_{p}\right| f\right\rangle \\
& =\left\langle d \uparrow\left|H_{p}\right| f\right\rangle=\left\langle d \uparrow\left|\frac{\hbar}{m_{0}}\left(\overline{\mathbf{k}} \cdot\left(\boldsymbol{\pi}+\hbar \mathbf{k}_{X}\right)+\frac{\hbar}{2} \bar{k}^{2}\right)\right| f\right\rangle \\
& =\frac{\hbar}{m_{0}} \overline{\mathbf{k}} \cdot\langle d \uparrow|\boldsymbol{\pi}| f\rangle
\end{aligned}
$$

Putting this all together equation (2.1) now looks like

$$
H_{X}=\left[\begin{array}{ccc}
E_{d \downarrow}(0)+\frac{\hbar^{2}}{2 m_{d \downarrow}} \bar{k}^{2} & \mathcal{S} . \mathcal{O} . & \mathcal{S} . \mathcal{O} . \\
\mathcal{S} . \mathcal{O} . & E_{d \uparrow}(0)+\frac{\hbar^{2}}{2 m_{d \uparrow}} \overline{k^{2}} & \frac{\hbar}{m_{0}} \overline{\mathbf{k}} \cdot\langle d \uparrow|\boldsymbol{\pi}| f\rangle \\
\mathcal{S} . \mathcal{O} . & \frac{\hbar}{m_{0}} \overline{\mathbf{k}} \cdot\langle f|\boldsymbol{\pi}| d \uparrow\rangle & E_{f b}(0)+\frac{\hbar^{2}}{2 m_{f b}} \bar{k}^{2}
\end{array}\right]
$$

However, we are also assuming that there is significant spin-splitting in SmN's energy bands due to the broken symmetry of the $4 f$ electrons below the Fermi level. From this we also then expect that any contributions from the spin-minority $5 d$ band will be negligible since the spin splitting will make any spin-orbit contributions negligible. Continuing on, for the sake of simplifying the notation, we will now drop the bar from $\overline{\mathbf{k}}$ so that $\mathbf{k}$ is now the wavevector measured from the $X$-point. We expect now the existence of pairing for superconductivity to occur in the two-band 
system of the majority-spin $4 f$ and $5 d$ bands like so:

$$
H_{N}(\mathbf{k})=\left[\begin{array}{cc}
\varepsilon_{d, \mathbf{k}} & \mathbf{k} \cdot \mathbf{P} \\
\mathbf{k} \cdot \mathbf{P}^{*} & \varepsilon_{f b, \mathbf{k}}
\end{array}\right]
$$

where $\varepsilon_{d, \mathbf{k}}=E_{d \uparrow}(0)+\frac{\hbar^{2}}{2 m_{d \uparrow}} k^{2}, \varepsilon_{f b, \mathbf{k}}=E_{f b}(0)+\frac{\hbar^{2}}{2 m_{f b}} k^{2}$, and $\mathbf{P}=\frac{\hbar}{m_{0}}\langle d \uparrow|\boldsymbol{\pi}| f\rangle$. For simplicity's sake, as in both (1.3) and (1.14), we will also measure the band dispersions with respect to the chemical potential $\mu$. As well as this we will assume that

\subsection{The Electron-Phonon Interaction}

As in BCS theory we assume that the interaction that mediates superconductivity is the electron-phonon interaction. The electron-phonon interaction is a process whereby two electrons can transfer momentum via a phonon wave in the material. In normal BCS superconductivity the effect provides a positive interaction between two electrons with equal and opposite momenta and spin to be linked to form the Cooper pairs upon which superconductivity operates. For more exotic pairing such as $p$-wave pairing, there have been different mechanisms proposed for the attractive interaction between electrons, for example spin fluctuations [92]. However, for our case to simplify matters we will consider the effective interaction to be due to phonons. In the same vein as BCS theory then we expect a similar formalism for this effect between electrons in our two-band system. For this model we will only consider long wavelength acoustic phonons as it will simplify the considerations we need to make for the effective potential.

We begin by considering the changes that an incoming phonon will have on a materials energy dispersion. Now informed by arguments made by Kittel [93] we write the deformation of the energy dispersions due to long wavelength acoustic phonons as:

$$
\varepsilon_{n}(\mathbf{k}, \mathbf{r})=\varepsilon_{0, n}(\mathbf{k})+C_{1, n} \Delta(\mathbf{r})
$$

with $\varepsilon_{0}$ being the unperturbed energy dispersions, $C_{1, n}=\left.\frac{\partial \varepsilon_{0, n}}{\partial \Delta}\right|_{\Delta=0}$ a constant, and the dilation of the lattice, $\Delta$, which is expressed as

$$
\Delta(\mathbf{r})=i \sum_{\mathbf{q}} \frac{1}{\sqrt{2 \rho \omega_{\mathbf{q}}}}|\mathbf{q}|\left(b_{\mathbf{q}} e^{i \mathbf{q} \cdot \mathbf{r}}-b_{\mathbf{q}}^{\dagger} e^{-i \mathbf{q} \cdot \mathbf{r}}\right)
$$

where $\rho$ is the density, $\omega_{\mathbf{q}}$ is the phonon dispersion, and $b_{\mathbf{q}}^{(\dagger)}$ is the phonon annihilation (creation) operator. We now consider the electron-phonon vertex as seen in figure 2.5 
where either a phonon is being absorbed or emitted from the scattering of an electron from a state $|n, \mathbf{k}\rangle$ to $\left|n^{\prime}, \mathbf{k}^{\prime}\right\rangle$. We may write this vertex as an overlap integral of

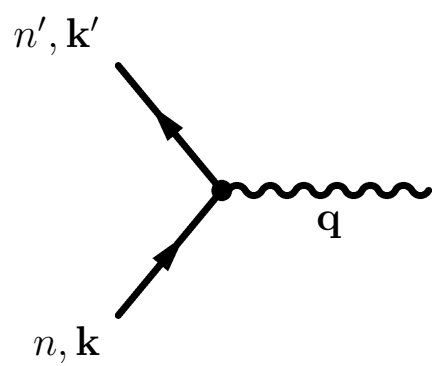

Figure 2.5: Vertex component for the electron-phonon interaction.

the form:

$$
A_{n, n^{\prime}}\left(\mathbf{k}, \mathbf{k}^{\prime}\right)=\int d r^{3} \psi_{n^{\prime} \mathbf{k}^{\prime}}^{\dagger}(\mathbf{r}) C_{1, n} \Delta(\mathbf{r}) \psi_{n \mathbf{k}}(\mathbf{r})
$$

To compute this integral we first recall equation (1.23) where we express the wavefunctions $\psi$ in terms of Bloch states. Since we also work within the $\mathbf{k} \cdot \mathbf{P}$ scheme we may also express our Bloch states perturbatively as we have done with our energy dispersions $(2.5,2.6)$, i.e. to first order

$$
u_{n \mathbf{k}}=u_{n \mathbf{k}_{X}}+\frac{\hbar}{m_{0}} \sum_{n^{\prime} \neq d \uparrow, f} \frac{\left\langle n|\mathbf{k} \cdot \boldsymbol{\pi}| n^{\prime}\right\rangle}{E_{n}(0)-E_{n^{\prime}}(0)} u_{n^{\prime} \mathbf{k}_{X}}
$$

where $\mathbf{k}$ is measured from the $X$-point. Since we are only interested in the $d$ and $f$ bands at the moment we may approximate the Bloch states then by $u_{n \mathbf{k}} \approx u_{n \mathbf{k}_{X}}$. We now may express $A_{n, n^{\prime}}\left(\mathbf{k}, \mathbf{k}^{\prime}\right)$ in the following way:

$$
\begin{aligned}
A_{n, n^{\prime}}\left(\mathbf{k}, \mathbf{k}^{\prime}\right)= & i C_{1, n} \sum_{\mathbf{q}} \frac{1}{\sqrt{2 \rho \omega_{\mathbf{q}}}}|\mathbf{q}| \\
& \times \int d r^{3} u_{n^{\prime} \mathbf{k}_{X}}^{*} u_{n \mathbf{k}_{X}}\left(b_{\mathbf{q}} e^{i\left(\mathbf{k}-\mathbf{k}^{\prime}+\mathbf{q}\right) \cdot \mathbf{r}}-b_{\mathbf{q}}^{\dagger} e^{i\left(\mathbf{k}-\mathbf{k}^{\prime}-\mathbf{q}\right) \cdot \mathbf{r}}\right) c_{n^{\prime}, \mathbf{k}^{\prime}}^{\dagger} c_{n, \mathbf{k}}
\end{aligned}
$$

Now since the Bloch functions are also periodic in the lattice then the factors $e^{i\left(\mathbf{k}-\mathbf{k}^{\prime} \pm \mathbf{q}\right) \cdot \mathbf{r}}$ in the integral restrict the wavevectors to obey $\mathbf{k}-\mathbf{k}^{\prime} \pm \mathbf{q}=0$ or $\mathbf{G}$ ( $\mathbf{G}$ a reciprocal lattice vector). If we have that $\mathbf{k}-\mathbf{k}^{\prime} \pm \mathbf{q}=0$ then the wavelike portion of the integral becomes a constant. On the other hand if $\mathbf{k}-\mathbf{k}^{\prime} \pm \mathbf{q}=\mathbf{G}$ then this portion of the integral remains non-constant. However, since we are in the long wavelength limit we may approximate the wavelike portion of the integral in this case to be constant over the unit cell to simplify the integral. Now we may treat the $u_{n^{\prime} \mathbf{k}_{X}}^{*} u_{n \mathbf{k}_{X}}$ term separately. Due to the orthogonality of the $u_{n, \mathbf{k}}$ Bloch functions (1.24) we may evaluate the integral $\int d r^{3} u_{n^{\prime} \mathbf{k}_{X}}^{*} u_{n \mathbf{k}_{X}}$ to be $\delta_{n, n^{\prime}}$ since we are considering, in the spirit of the $\mathbf{k} \cdot \mathbf{P}$ method, the $u_{n, \mathbf{k}}$ only at the $X$ point. 


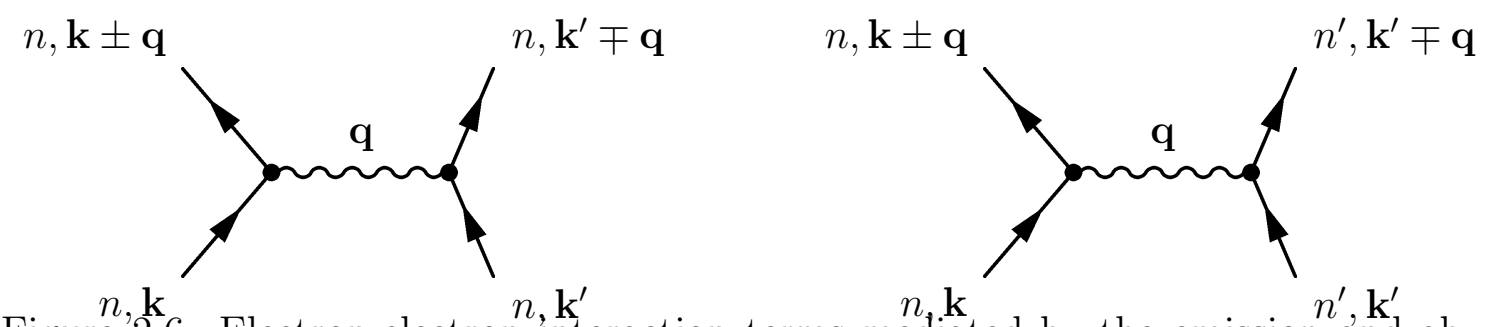

Figure 2.6: Electron-electron interaction terms mediated by the emission and absorption of virtual phonons. The changed momenta $\mathbf{k}+(-) \mathbf{q}$ correspond to the absorption (emission) of a phonon.

Due to the periodicity of the Bloch functions this will be the same in every unit cell in the crystal. Now the remaining wavelike part of the integral may be integrated simply to unity as long the wavevector restrictions are observed. Thus we now write (2.14) as

$$
A_{n, n^{\prime}}\left(\mathbf{k}, \mathbf{k}^{\prime}\right)=i C_{1, n} \sum_{\mathbf{q}} \frac{1}{\sqrt{2 \rho \omega_{\mathbf{q}}}}|\mathbf{q}| \delta_{n, n^{\prime}}\left(b_{\mathbf{q}} \delta_{\mathbf{k}^{\prime}-\mathbf{k}, \mathbf{q}}-b_{\mathbf{q}}^{\dagger} \delta_{\mathbf{k}-\mathbf{k}^{\prime}, \mathbf{q}}\right) c_{n^{\prime}, \mathbf{k}^{\prime}}^{\dagger} c_{n, \mathbf{k}}
$$

From the form of (2.15) we immediately see that the matrix element prohibits scattering events that shift an electron into a different energy band, i.e. the diagram in figure 2.5 may only scatter to the same band $\left(n=n^{\prime}\right)$. Therefore we can express $A_{n, n^{\prime}}$ as just $A_{n}$. We may simplify $A_{n}$ further by evaluating the $\mathbf{q}$ sum to gain

$$
A_{n}\left(\mathbf{k}, \mathbf{k}^{\prime}\right)=i C_{1, n} \frac{\left|\mathbf{k}^{\prime}-\mathbf{k}\right|}{\sqrt{2 \rho}}\left(\frac{1}{\sqrt{\omega_{\mathbf{k}^{\prime}-\mathbf{k}}}} b_{\mathbf{k}^{\prime}-\mathbf{k}}-\frac{1}{\sqrt{\omega_{\mathbf{k}-\mathbf{k}^{\prime}}}} b_{\mathbf{k}-\mathbf{k}^{\prime}}^{\dagger}\right) c_{n, \mathbf{k}^{\prime}}^{\dagger} c_{n, \mathbf{k}}
$$

since $\left|\mathbf{k}^{\prime}-\mathbf{k}\right|=\left|\mathbf{k}-\mathbf{k}^{\prime}\right|$. This model as described by Kittel [93] is also only valid for acoustic phonons since the dilation is only related to acoustic phonons and that we have not included long-range electrostatic potentials which would arise from optical phonon deformations. Therefore the phonon frequencies $\omega_{\mathbf{q}}$ can be expressed as:

$$
\omega_{\mathbf{q}}=v_{s}|\mathbf{q}|
$$

where $v_{s}$ is the speed of sound in the material. Now we may write $A_{n}$ as

$$
A_{n}\left(\mathbf{k}, \mathbf{k}^{\prime}\right)=i C_{1, n} \frac{\left|\mathbf{k}^{\prime}-\mathbf{k}\right|}{\sqrt{2 \rho \omega_{\mathbf{k}^{\prime}-\mathbf{k}}}}\left(b_{\mathbf{k}^{\prime}-\mathbf{k}}-b_{\mathbf{k}-\mathbf{k}^{\prime}}^{\dagger}\right) c_{n, \mathbf{k}^{\prime}}^{\dagger} c_{n, \mathbf{k}}
$$

where we can now identify the prefactor that will give us the strength of the electronphonon interaction:

$$
M_{n}\left(\mathbf{k}^{\prime}-\mathbf{k}\right)=i C_{1, n} \frac{\left|\mathbf{k}^{\prime}-\mathbf{k}\right|}{\sqrt{2 \rho \omega_{\mathbf{k}^{\prime}-\mathbf{k}}}}
$$

We note that this is based on the assumption that both the $d$ and $f$ bands can 
be treated with the Born-Oppenhiemer adiabatic approximation, which may not be the case for the $f$ electrons due to their highly atomic-like behaviour and slow group velocity due to their flat dispersion.

The interaction terms themselves will be composed of two vertices as we see from the Feynman diagrams (figure 2.6). This means that while a single vertex cannot swap band indices the full interaction does not prohibit joining two vertices with different band indices together. So now if we were to have an interaction term between electrons in bands $n$ and $n^{\prime}$ we would be able to write the interaction term as

$$
V_{n, n^{\prime}}=M_{n} M_{n^{\prime}}^{*}=C_{1, n} C_{1, n^{\prime}} \frac{\left(\mathbf{k}^{\prime}-\mathbf{k}\right)^{2}}{2 \rho \omega_{\mathbf{k}^{\prime}-\mathbf{k}}}
$$

If we also use the expression for the phonon frequencies (2.17) then we may write

$$
V_{n, n^{\prime}}=\frac{C_{1, n} C_{1, n^{\prime}}}{2 \rho v_{s}}\left|\mathbf{k}^{\prime}-\mathbf{k}\right|=V\left|\mathbf{k}^{\prime}-\mathbf{k}\right|
$$

We see here that the relative strength of these interactions are purely based on the constants $C_{1, n}$ and that the interaction will depend on $\left|\mathbf{k}^{\prime}-\mathbf{k}\right|$ which is even in $\left(\mathbf{k}^{\prime}-\mathbf{k}\right)$. 


\section{Chapter 3}

\section{Triplet Pairing Superconductivity in $\mathrm{SmN}$}

\subsection{The Effective Hamiltonian}

We begin our investigation into superconductivity in $\mathrm{SmN}$ by first considering the normal state Hamiltonian of our system (2.9) which we will rewrite in the following way:

$$
H_{N}(\mathbf{k})=\left[\begin{array}{cc}
\varepsilon_{d, \mathbf{k}} & e^{i \theta} \mathbf{k} \cdot \mathbf{P} \\
e^{-i \theta} \mathbf{k} \cdot \mathbf{P} & \varepsilon_{f b}
\end{array}\right]
$$

where now we have made explicit the (possible) complex nature of the $\mathbf{k} \cdot \mathbf{P}$ term through the introduction of the phase factor $e^{i \theta}$. As explained before this Hamiltonian will describe the two-band system close to the Fermi level. The $5 d$ band here will have a quadratic dispersion while we assume that the $4 f$ band will be dispersionless since the $4 f$ band hosts highly localised electrons which orbit close to the nucleus of the Sm atom. The off-diagonal elements in (3.1) will act as a term to introduce band hybridisation into the system. Band hybridisation is when one band mixes with another such that the new hybridised band now has characteristics of both of the unmixed bands. The hybridisation serves also to introduce avoided crossings at the points where the two bands would normally intersect. As an example, the dispersions in our case gives new hybridised bands of the form seen in figure 3.1. We see that instead of the $4 f$ band intersecting the $5 d$ band as in figure 2.3 the bands form an avoided crossing such that the states do not overlap. Such hybridisation between the bands can even be seen in the calculated bandstructure of $\mathrm{SmN}$ in figure 2.3 where the red of the $4 f$ bands give way to the blue of the $5 d$ band and vice versa.

We wish to add to the Hamiltonian (3.1) the phonon interaction terms that 


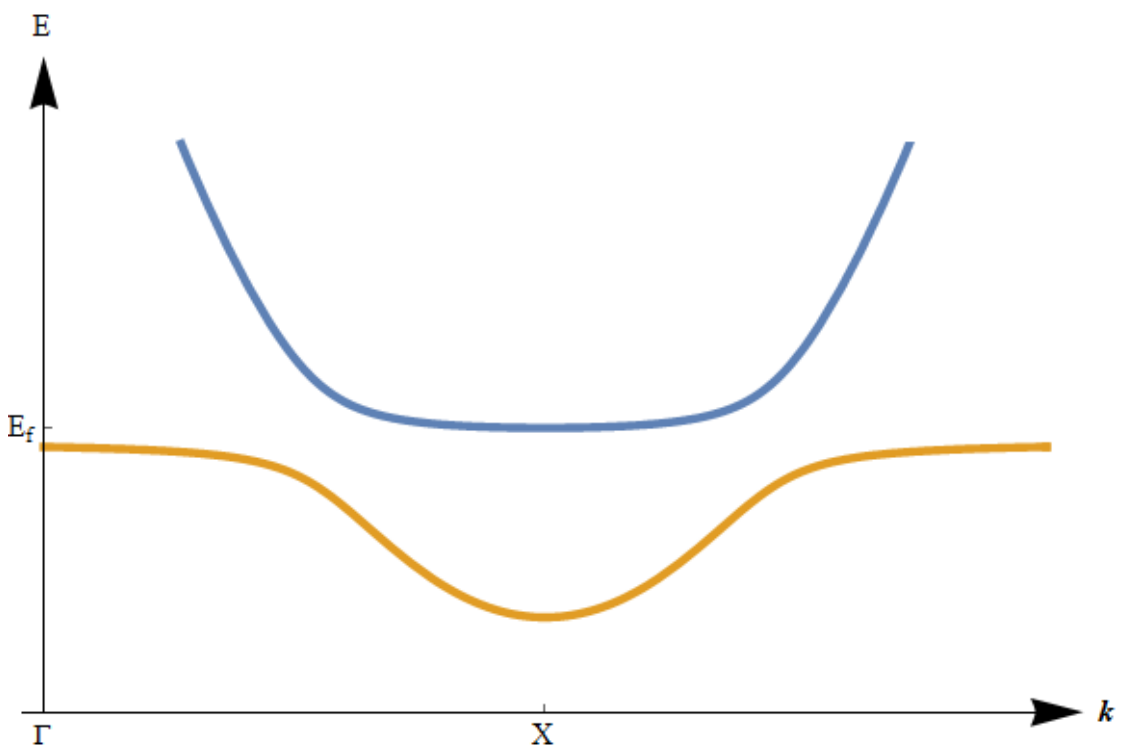

Figure 3.1: A plot of how the two energy bands $\varepsilon_{d, \mathbf{k}}$ and $\varepsilon_{f b}$ will hybridise with each other. The presence of the off diagonal terms (non-zero $\mathbf{k} \cdot \mathbf{P}$ ) causes the hybridization of the bands. The functional forms of these bands can be recovered by diagonalising (3.1).

will lead to the superconductivity in SmN. We do this by writing (3.1) in the second quantisation formalism as we have seen in (1.1) and (1.12). To do this we are guided by a previous $s$-wave two-band Hamiltonian by Kristoffel et al. [94] and we construct our Hamiltonian in a similar way. The Hamiltonian of Ref [94] includes terms that pairs electrons in the same band (intra-band pairing). However, unlike this previous Hamiltonian we are in a spin polarised system and so we may drop the spin degree of freedom. We will also add in another term that forms pairings between electrons in different bands (inter-band pairing) so that the Hamiltonian includes all possible pairing combinations that could exist in the system. The resultant Hamiltonian reads:

$$
\begin{aligned}
H= & \sum_{n, \mathbf{k}} \varepsilon_{n, \mathbf{k}} a_{n, \mathbf{k}}^{\dagger} a_{n, \mathbf{k}}+\sum_{\mathbf{k}}\left[\mathbf{k} \cdot \mathbf{P} e^{i \theta} a_{d, \mathbf{k}}^{\dagger} a_{f b, \mathbf{k}}+\text { H.c. }\right] \\
& +\frac{1}{2} \sum_{n, \mathbf{k}, \mathbf{k}^{\prime}} V_{n n}\left(\mathbf{k}, \mathbf{k}^{\prime}\right) a_{n, \mathbf{k}}^{\dagger} a_{n,-\mathbf{k}}^{\dagger} a_{n,-\mathbf{k}^{\prime}} a_{n, \mathbf{k}^{\prime}} \\
& +\sum_{\mathbf{k}, \mathbf{k}^{\prime}} V_{d f}\left(\mathbf{k}, \mathbf{k}^{\prime}\right) a_{d, \mathbf{k}}^{\dagger} a_{f b,-\mathbf{k}}^{\dagger} a_{f b,-\mathbf{k}^{\prime}} a_{d, \mathbf{k}^{\prime}} \\
& +\sum_{\mathbf{k}, \mathbf{k}^{\prime}} V_{d f}^{\prime}\left(\mathbf{k}, \mathbf{k}^{\prime}\right)\left[a_{d, \mathbf{k}}^{\dagger} a_{d,-\mathbf{k}}^{\dagger} a_{f b,-\mathbf{k}^{\prime}} a_{f b, \mathbf{k}^{\prime}}+a_{f b, \mathbf{k}}^{\dagger} a_{f b,-\mathbf{k}}^{\dagger} a_{d,-\mathbf{k}^{\prime}} a_{d, \mathbf{k}^{\prime}}\right]
\end{aligned}
$$

where $n$ is the band index (either $d$ or $f b$ ), $\varepsilon_{n, \mathbf{k}}$ is the energy dispersion for each band, $V_{n n^{\prime}}\left(\mathbf{k}, \mathbf{k}^{\prime}\right)$ is the matrix element for pairing between bands $n$ and $n^{\prime}, a_{n, \mathbf{k}}^{(\dagger)}$ is 
the annihilation (creation) operator for an electron in band $n$ with momentum $\mathbf{k}$, and H.c. stands for the Hermitian conjugate. In (3.2) we note that the interaction terms here are positive which generally indicate repulsive interactions. While this is in contrast to the Hamiltonians in (1.1) and (1.12) we shall see later that the specific choice of potential (2.21) will give rise to an attractive interaction.

The first line of the Hamiltonian is merely a rewriting of the matrix (3.1) while the second and third lines describe intra- and inter-band electron pairing. The final line however we immediately recognise as a pairing that scatters paired electrons from one band into the other which, by the result (2.15) in chapter 2, we know is forbidden. Hence we may remove this term entirely from consideration and rewrite our second quantisation Hamiltonian as

$$
\begin{aligned}
H= & \sum_{n, \mathbf{k}} \varepsilon_{n, \mathbf{k}} a_{n, \mathbf{k}}^{\dagger} a_{n, \mathbf{k}}+\sum_{\mathbf{k}}\left[\mathbf{k} \cdot \mathbf{P} e^{i \theta} a_{d, \mathbf{k}}^{\dagger} a_{f b, \mathbf{k}}+\text { H.C. }\right] \\
& +\frac{1}{2} \sum_{n, \mathbf{k}, \mathbf{k}^{\prime}} V_{n n}\left(\mathbf{k}, \mathbf{k}^{\prime}\right) a_{n, \mathbf{k}}^{\dagger} a_{n,-\mathbf{k}}^{\dagger} a_{n,-\mathbf{k}^{\prime}} a_{n, \mathbf{k}^{\prime}} \\
& +\sum_{\mathbf{k}, \mathbf{k}^{\prime}} V_{d f}\left(\mathbf{k}, \mathbf{k}^{\prime}\right) a_{d, \mathbf{k}}^{\dagger} a_{f b,-\mathbf{k}}^{\dagger} a_{f b,-\mathbf{k}^{\prime}} a_{d, \mathbf{k}^{\prime}}
\end{aligned}
$$

As said before the second line describes intra-band pairing which pairs two electrons in the same band together into a Cooper pair. We see that the electrons in this case must both have the same spin (spin up in this case) and therefore must have even spin parity. This means that this pairing must be of triplet nature (as in fig 1.3) which we likely suspect to be $p$-wave since it is the leading term $(l=1)$ in an odd expansion in spherical harmonics (see table 1.1). This term, then, leads to $p$-wave pairing in both the $f$ and $d$ bands. The third term on the other hand describes inter-band pairing; pairing between two electrons in different bands. The form of such a pairing is evocative due to the structure of its band indices. If one compares this term to the one in equation (1.1) we can immediately see the similarity of the terms by substituting the band indices with differing spins. The form of this pairing means that now we can construct a pairing that has triplet pairing in the spins but singlet pairing in the band indices. In this case, now, such a term will describe an overall singlet pairing in the material, indeed such a possibility is one of the enticing reasons for considering multi-band superconductivity in the first place.

While the singlet pairing term is appealing due to the predisposition of such symmetries surviving random non-magnetic impurities we must first look to the specific form of the interaction that we have developed in the preceding chapter. Equation (2.21) clearly shows that the strength of the electron-phonon interaction 
will be dependent on the constants $C_{1, n}$. Remembering the specific form of these constants $C_{1, n}=\left.\frac{\partial \varepsilon_{0, n}}{\partial \Delta}\right|_{\Delta=0}$ we see that the constant is dependent on the change in the energy dispersion with respect to the dilation of the lattice i.e. how much will the energy dispersion be deformed by some change in the lattice. Considering now the relative strengths of the $C_{1, n}$ for the $d$ and $f$ bands we expect the coupling strength for the $f$ electrons to be smaller than that of the $d$ electrons. As well as reported small $4 f$ coupling strength in Sm compared to other RE elements [86], our physical reasoning for this conclusion is that the phonons mediating this process are created via the application of stress to the crystal which in turn changes the atomic coordinates of the crystal. Since the $4 f$ electrons are highly localised and orbit close to the nucleus of the samarium atoms then some shift in atomic coordinates will not impact the energy dispersions of these states as they will simply move with the atom as the phonon passes through the crystal. Compared to the $4 f$ band, the $5 d$ band will be more affected by the compression and expansion of the crystal since the broad dispersion of the $5 d$ band is spread throughout the crystal and dependent on the periodicity of the lattice. The alterations to the periodicity of the lattice, caused by the passage of the phonon, will thus disrupt this broad dispersion. Therefore we will find that the constants controlling the strength of the phonon interaction are such that $C_{1, d} \gg C_{1, f}$ in this case. Thus we may take the potentials $V_{f f}$ and $V_{d f}$ to be negligible compared to the $V_{d d}$ term. This leads us to discarding the singlet term in (3.3) and the triplet term for the $f$ band and allows us to write our Hamiltonian simply as

$$
\begin{aligned}
H= & \sum_{n, \mathbf{k}} \varepsilon_{n, \mathbf{k}} a_{n, \mathbf{k}}^{\dagger} a_{n, \mathbf{k}}+\sum_{\mathbf{k}}\left[\mathbf{k} \cdot \mathbf{P} e^{i \theta} a_{d, \mathbf{k}}^{\dagger} a_{f b, \mathbf{k}}+\text { H.C. }\right] \\
& +\frac{1}{2} \sum_{\mathbf{k}, \mathbf{k}^{\prime}} V_{d d}\left(\mathbf{k}^{\prime}-\mathbf{k}\right) a_{d, \mathbf{k}}^{\dagger} a_{d,-\mathbf{k}}^{\dagger} a_{d,-\mathbf{k}^{\prime}} a_{d, \mathbf{k}^{\prime}}
\end{aligned}
$$

This means we will now only consider triplet pairing of the electrons in the $d$ band.

\subsection{The Mean Field Hamiltonian}

In order to find the gap equation for for the Hamiltonian (3.4) we will use the mean field approximation as in sections subsection 1.2.1 and subsection 1.2.3. As before we will construct the mean field Hamiltonian by introducing the pair potential $\Delta_{d}$ which we define as

$$
\Delta_{d}(\mathbf{k})=-\sum_{\mathbf{k}^{\prime}} V_{d d}\left(\mathbf{k}, \mathbf{k}^{\prime}\right)\left\langle a_{d,-\mathbf{k}^{\prime}} a_{d, \mathbf{k}^{\prime}}\right\rangle
$$


The reason for defining the pair potential (3.5) with a minus sign will become obvious once we consider more carefully the specific form of $V_{d d}$. This lets us write the following mean field Hamiltonian:

$$
\begin{aligned}
H_{\mathrm{M} . \mathrm{F} .}= & \sum_{n, \mathbf{k}} \varepsilon_{n, \mathbf{k}} a_{n, \mathbf{k}}^{\dagger} a_{n, \mathbf{k}}+\sum_{\mathbf{k}}\left[\mathbf{k} \cdot \mathbf{P} e^{i \theta} a_{d, \mathbf{k}}^{\dagger} a_{f b, \mathbf{k}}+\text { H.C. }\right] \\
& -\frac{1}{2} \sum_{\mathbf{k}}\left[\Delta_{d, \mathbf{k}} a_{d, \mathbf{k}}^{\dagger} a_{d,-\mathbf{k}}^{\dagger}+\text { H.C. }\right]
\end{aligned}
$$

We may also rewrite this Hamiltonian using Nambu notation to express it as a matrix. Writing the Nambu spinors as $\vec{a}_{\mathbf{k}}^{\dagger}=\left[a_{d, \mathbf{k}}^{\dagger}, a_{f b, \mathbf{k}}^{\dagger}, a_{d,-\mathbf{k}}, a_{f b,-\mathbf{k}}\right]$ the Hamiltonian (3.6) can be rewritten as

$$
H_{M . F .}=\frac{1}{2} \sum_{\mathbf{k}} \vec{a}_{\mathbf{k}}^{\dagger}\left[\begin{array}{cccc}
\varepsilon_{d, \mathbf{k}} & e^{i \theta} \mathbf{k} \cdot \mathbf{P} & -\Delta_{d, \mathbf{k}} & 0 \\
e^{-i \theta} \mathbf{k} \cdot \mathbf{P} & \varepsilon_{f b} & 0 & 0 \\
-\Delta_{d, \mathbf{k}}^{*} & 0 & -\varepsilon_{d, \mathbf{k}} & e^{-i \theta} \mathbf{k} \cdot \mathbf{P} \\
0 & 0 & e^{i \theta} \mathbf{k} \cdot \mathbf{P} & -\varepsilon_{f b}
\end{array}\right] \vec{a}_{\mathbf{k}}
$$

Now that we have the Hamiltonian (3.7) in this form we can diagonalise this matrix with some unitary transformation so that in the end we may write and solve the self consistency equation for $\Delta_{d}$.

We now look into the form of the interaction term in more detail to uncover how we generate our attractive interaction from a seemingly repulsive interaction term. We begin with some potential (suppressing spin indices since we are still in a spin polarised system) in the usual way

$$
V=\frac{1}{2} \sum_{\mathbf{k}, \mathbf{k}^{\prime}, \mathbf{q}} V(\mathbf{q}) c_{\mathbf{k}^{\prime}+\mathbf{q}}^{\dagger} c_{\mathbf{k}-\mathbf{q}}^{\dagger} c_{\mathbf{k}} c_{\mathbf{k}^{\prime}}
$$

This equation describes a process that imparts some momentum q to an electron at the expense of another electron. We then would like to set up paired electrons of opposite momenta, so we take $\mathbf{k}=-\mathbf{k}^{\prime}$ which gives:

$$
\begin{aligned}
V & =\frac{1}{2 V o l} \sum_{\mathbf{k}^{\prime}, \mathbf{q}} V(\mathbf{q}) c_{\mathbf{k}^{\prime}+\mathbf{q}}^{\dagger} c_{-\mathbf{k}^{\prime}-\mathbf{q}}^{\dagger} c_{-\mathbf{k}^{\prime}} c_{\mathbf{k}^{\prime}} \\
& =\frac{1}{2 V o l} \sum_{\mathbf{k}, \mathbf{k}^{\prime}} V\left(\mathbf{k}-\mathbf{k}^{\prime}\right) c_{\mathbf{k}}^{\dagger} c_{-\mathbf{k}^{\dagger}}^{\dagger} c_{-\mathbf{k}^{\prime}} c_{\mathbf{k}^{\prime}}
\end{aligned}
$$

where in the second equality we have taken $\mathbf{q}=\mathbf{k}-\mathbf{k}^{\prime}$. Until this point we have said nothing about the nature of this potential but now we assume that the potential is 
repulsive, like that of Coulomb repulsion or phonon coupling. Now from (2.21) we have that $V\left(\mathbf{k}-\mathbf{k}^{\prime}\right)=V\left|\mathbf{k}-\mathbf{k}^{\prime}\right|$ where $V$ is positive and so $V\left(\mathbf{k}-\mathbf{k}^{\prime}\right)$ is always positive, i.e. the potential is repulsive. However, what we may do is expand $V\left(\mathbf{k}-\mathbf{k}^{\prime}\right)$ in the following way so that

$$
\begin{aligned}
V\left(\mathbf{k}-\mathbf{k}^{\prime}\right) & =V\left|\mathbf{k}-\mathbf{k}^{\prime}\right| \approx V_{0}+V_{2}\left(\mathbf{k}-\mathbf{k}^{\prime}\right)^{2}+\mathcal{O}\left[\left(\mathbf{k}-\mathbf{k}^{\prime}\right)^{2}\right] \\
& =V_{0}+V_{2}\left(k^{2}+k^{\prime 2}\right)-2 V_{2} \mathbf{k} \cdot \mathbf{k}^{\prime}+\mathcal{O}\left[\left(\mathbf{k}-\mathbf{k}^{\prime}\right)^{2}\right]
\end{aligned}
$$

Now we see that from a purely repulsive potential we find that there is an attractive component of the potential. In normal BCS theory this repulsive component of the potential is usually cancelled out during the necessary operator algebra and we need only to keep the surviving constant terms. However, in the case of the triplet pairing we do not have the same result, in fact if we take the potential to be the leading constant term then we would find that our pairing potential is zero. Performing operator algebra with the specific form of $V_{d d}(2.21)$ on the pair potential as defined in $(3.5)$ we find

$$
\Delta_{d}(\mathbf{k})=\frac{V}{2}\left[\sum_{\mathbf{k}^{\prime}}\left|\mathbf{k}-\mathbf{k}^{\prime}\right|\left\langle a_{d,-\mathbf{k}^{\prime}} a_{d, \mathbf{k}^{\prime}}\right\rangle+\sum_{\mathbf{k}^{\prime} \rightarrow-\mathbf{k}^{\prime}}\left|\mathbf{k}-\mathbf{k}^{\prime}\right|\left\langle a_{d,-\mathbf{k}^{\prime}} a_{d, \mathbf{k}^{\prime}}\right\rangle\right]
$$

where we have split the sum into two equal parts and are intending to send the second sum from $\mathbf{k}^{\prime} \rightarrow-\mathbf{k}^{\prime}$. By sending $\mathbf{k}^{\prime} \rightarrow-\mathbf{k}^{\prime}$ in the second sum we are able to write now:

$$
\Delta_{d}(\mathbf{k})=\frac{V}{2} \sum_{\mathbf{k}^{\prime}}\left[\left|\mathbf{k}-\mathbf{k}^{\prime}\right|\left\langle a_{d,-\mathbf{k}^{\prime}} a_{d, \mathbf{k}^{\prime}}\right\rangle+\left|\mathbf{k}+\mathbf{k}^{\prime}\right|\left\langle a_{d, \mathbf{k}^{\prime}} a_{d,-\mathbf{k}^{\prime}}\right\rangle\right]
$$

Now by making use of the fact that the anticommutater of two different fermion operators is zero (i.e. $\left\{a_{\alpha}, a_{\beta}\right\}=\delta_{\alpha \beta}$ ) we may make the swap $\left\langle a_{d, \mathbf{k}^{\prime}} a_{d,-\mathbf{k}^{\prime}}\right\rangle=-\left\langle a_{d,-\mathbf{k}^{\prime}} a_{d, \mathbf{k}^{\prime}}\right\rangle$ in (3.12). This allows to write $\Delta_{d}(\mathbf{k})$ in the following way:

$$
\Delta_{d}(\mathbf{k})=\frac{1}{2} \sum_{\mathbf{k}^{\prime}}\left[V\left(\left|\mathbf{k}-\mathbf{k}^{\prime}\right|-\left|\mathbf{k}+\mathbf{k}^{\prime}\right|\right)\right]\left\langle a_{d,-\mathbf{k}^{\prime}} a_{d, \mathbf{k}^{\prime}}\right\rangle
$$

The main takeaway from this new form of $\Delta_{d, \mathbf{k}}$ has the effective potential as $V_{d d}(\mathbf{k}-$ $\left.\mathbf{k}^{\prime}\right)-V_{d d}\left(\mathbf{k}+\mathbf{k}^{\prime}\right)$. Now connecting (3.10) to this result we see that $V_{d d}\left(\mathbf{k}-\mathbf{k}^{\prime}\right)-$ $V_{d d}\left(\mathbf{k}+\mathbf{k}^{\prime}\right)$ will be purely attractive as a potential since:

$$
V_{d d}\left(\mathbf{k}-\mathbf{k}^{\prime}\right)-V_{d d}\left(\mathbf{k}+\mathbf{k}^{\prime}\right) \approx-4 V_{2} \mathbf{k} \cdot \mathbf{k}^{\prime} .
$$


This is to be expected from Akhiezer et al. [95] since we are looking fundamentally at $p$-wave pairing in the $d$-band. Remembering that this came from a purely repulsive potential we now see why we have started with such a repulsive interaction term in our second quantisation Hamiltonian. We see now the reason for introducing a minus sign in the pair potential (3.5) as we may now modify the pair potential to have the following form:

$$
\begin{aligned}
\Delta_{d}(\mathbf{k}) & =-\frac{1}{2} \sum_{\mathbf{k}^{\prime}}\left[V_{d d}\left(\mathbf{k}-\mathbf{k}^{\prime}\right)-V_{d d}\left(\mathbf{k}+\mathbf{k}^{\prime}\right)\right]\left\langle a_{d,-\mathbf{k}^{\prime}} a_{d, \mathbf{k}^{\prime}}\right\rangle \\
& =2 V_{2} \sum_{\mathbf{k}^{\prime}} \mathbf{k} \cdot \mathbf{k}^{\prime}\left\langle a_{d,-\mathbf{k}^{\prime}} a_{d, \mathbf{k}^{\prime}}\right\rangle .
\end{aligned}
$$

We note that the constant $V_{2}$ will have units of energy per wavevector squared since it is a second order expansion of $V_{d d}\left(\mathbf{k}-\mathbf{k}^{\prime}\right)$. We have considered a model where the interaction term is mediated by phonons. However, the results which we will present in the following rely only on the property where $V_{d d}\left(\mathbf{k}-\mathbf{k}^{\prime}\right)-V_{d d}\left(\mathbf{k}+\mathbf{k}^{\prime}\right) \approx-4 V_{2} \mathbf{k} \cdot \mathbf{k}^{\prime}$, irrespective of how the interaction is mediated.

\subsubsection{Diagonalising the Mean-Field Hamiltonian}

We are now in a position to diagonalise the mean-field Hamiltonian (3.7). Considering just the matrix in the Hamiltonian (3.7) and calling it $\mathcal{H}_{\mathbf{k}}$ we wish to find a unitary matrix $U$ such that

$$
U^{\dagger} \mathcal{H}_{\mathbf{k}} U=\left[\begin{array}{cccc}
E_{1, \mathbf{k}} & 0 & 0 & 0 \\
0 & E_{2, \mathbf{k}} & 0 & 0 \\
0 & 0 & E_{3, \mathbf{k}} & 0 \\
0 & 0 & 0 & E_{4, \mathbf{k}}
\end{array}\right]=\mathcal{H}_{\mathbf{k}}^{\text {diag }}
$$

where the $E_{n, \mathbf{k}}, n=1, \ldots, 4$ are the eigenenergies of the new diagonalised system. The way to do this is of course the foundational theorem from linear algebra whereby if there are $n$ distinct eigenvalues of an $n \times n$ Hermitian matrix ( $E_{n, \mathbf{k}}$ in this case) then such a diagonalisation is possible where the columns of the required $U$ will be the eigenvectors associated with each eigenvalue ${ }^{1}$. Now all we need to do is introduce a substitution to give us a matrix product as in (3.16) which we do in the standard way. Since we have that $H_{M . F .} \propto \vec{a}_{\mathbf{k}}^{\dagger} \mathcal{H}_{\mathbf{k}} \vec{a}_{\mathbf{k}}$ then we can move to a new operator

\footnotetext{
${ }^{1}$ While it is true that not every eigenenergy from a Hamiltonian need be distinct we will not need to worry about such a case here.
} 
system, $\vec{\gamma}_{\mathbf{k}}$, with the following substitution:

$$
\left[\begin{array}{c}
a_{d, \mathbf{k}} \\
a_{f b, \mathbf{k}} \\
a_{d,-\mathbf{k}}^{\dagger} \\
a_{f b,-\mathbf{k}}^{\dagger}
\end{array}\right]=U\left[\begin{array}{c}
\gamma_{1, \mathbf{k}} \\
\gamma_{2, \mathbf{k}} \\
\gamma_{1,-\mathbf{k}}^{\dagger} \\
\gamma_{2,-\mathbf{k}}^{\dagger}
\end{array}\right]
$$

This substitution is valid since we can write:

$$
\begin{gathered}
H_{\text {M.F. }}=\frac{1}{2} \sum_{\mathbf{k}} \vec{a}_{\mathbf{k}}^{\dagger} \mathcal{H}_{\mathbf{k}} \vec{a}_{\mathbf{k}} \\
=\frac{1}{2} \sum_{\mathbf{k}} \vec{\gamma}_{\mathbf{k}}^{\dagger} U^{\dagger} \mathcal{H}_{\mathbf{k}} U \vec{\gamma}_{\mathbf{k}} \\
=\frac{1}{2} \sum_{\mathbf{k}} \vec{\gamma}_{\mathbf{k}}^{\dagger} \mathcal{H}_{\mathbf{k}}^{\text {diag }} \vec{\gamma}_{\mathbf{k}}
\end{gathered}
$$

which is what we require. As stated before the $U$ we require will be of the form

$$
U=\left[\begin{array}{llll}
\mathbf{w}_{1, \mathbf{k}}, & \mathbf{w}_{2, \mathbf{k}}, & \mathbf{w}_{3, \mathbf{k}}, & \mathbf{w}_{4, \mathbf{k}}
\end{array}\right]
$$

where the $\mathbf{w}_{n, \mathbf{k}}$ are the associated eigenspinors of the eigenenergies $E_{n, \mathbf{k}}$. It will also be useful later to define now the eigenspinors as having the following form:

$$
\mathbf{w}_{n, \mathbf{k}}=\left[u_{1, n, \mathbf{k}}, \quad u_{2, n, \mathbf{k}}, \quad v_{1, n, \mathbf{k}}, \quad v_{2, n, \mathbf{k}}\right]^{T}
$$

We are now in a position to diagonalise (3.7) which can be done straightforwardly to give the eigenenergies:

$$
\begin{gathered}
E_{1}(\mathbf{k})=\frac{1}{\sqrt{2}} \sqrt{2(\mathbf{k} \cdot \mathbf{P})^{2}+\varepsilon_{d, \mathbf{k}}^{2}+\varepsilon_{f b}^{2}+\left|\Delta_{d}\right|^{2}+b_{\mathbf{k}}} \\
E_{2}(\mathbf{k})=\frac{1}{\sqrt{2}} \sqrt{2(\mathbf{k} \cdot \mathbf{P})^{2}+\varepsilon_{d, \mathbf{k}}^{2}+\varepsilon_{f b}^{2}+\left|\Delta_{d}\right|^{2}-b_{\mathbf{k}}} \\
E_{3}(\mathbf{k})=-E_{1}(\mathbf{k}) \\
E_{4}(\mathbf{k})=-E_{2}(\mathbf{k})
\end{gathered}
$$

where $b_{\mathbf{k}}$ is defined as

$$
b_{\mathbf{k}}=\sqrt{\left(\varepsilon_{d, \mathbf{k}}^{2}-\varepsilon_{f b}^{2}+\left|\Delta_{d}\right|^{2}\right)^{2}+4(\mathbf{k} \cdot \mathbf{P})^{2}\left(\left|\Delta_{d}\right|^{2}+\left(\varepsilon_{d, \mathbf{k}}+\varepsilon_{f b}\right)^{2}\right)}
$$


With this we have successfully rewritten $H_{M . F}$. into the expected form as we have seen previously i.e.

$$
H_{M . F .}=\sum_{n, \mathbf{k}} E_{n, \mathbf{k}} \gamma_{n, \mathbf{k}}^{\dagger} \gamma_{n, \mathbf{k}}
$$

where $n=1,2$. This leads us to our guess for the groundstate of the system which we write as:

$$
\left|\Phi_{\text {G.S. }}\right\rangle=\prod_{\mathbf{k}} \gamma_{1, \mathbf{k}} \gamma_{2, \mathbf{k}}\left|\Phi_{0}\right\rangle
$$

with $\left|\Phi_{0}\right\rangle$ indicating electronic vacuum. This state satisfies the necessary condition for the groundstate since $\gamma_{n, \mathbf{k}}\left|\Phi_{\text {G.S. }}\right\rangle=0$ for $n=1,2$ and any $\mathbf{k}$. In the interests of saving space the eigenspinors $\mathbf{w}_{n}$ will not be written out here as we will discover that we will only require two of the four for expressing the pair potential in the self consistency equation. To discover which of these we require we will need to set up the self consistency equation for $\Delta_{d}$ which we do in following section.

\subsection{Self Consistency Equation for $\Delta_{d}$}

In order for us to write the self consistency equation for $\Delta_{d}$ we must first find out how to express the expectation value $\left\langle a_{d,-\mathbf{k}^{\prime}} a_{d, \mathbf{k}^{\prime}}\right\rangle$ in terms of the $\gamma_{n, \mathbf{k}}$ operators. Using (3.17) in conjunction with (3.19) and (3.20) we are able to simply write the $a$ operators in terms of the $\gamma$ operators:

$$
\begin{aligned}
a_{d, \mathbf{k}} & =u_{1,1, \mathbf{k}} \gamma_{1, \mathbf{k}}+u_{1,2, \mathbf{k}} \gamma_{2, \mathbf{k}}+u_{1,3, \mathbf{k}} \gamma_{1,-\mathbf{k}}^{\dagger}+u_{1,4, \mathbf{k}} \gamma_{2,-\mathbf{k}}^{\dagger} \\
a_{f b, \mathbf{k}} & =u_{2,1, \mathbf{k}} \gamma_{1, \mathbf{k}}+u_{2,2, \mathbf{k}} \gamma_{2, \mathbf{k}}+u_{2,3, \mathbf{k}} \gamma_{1,-\mathbf{k}}^{\dagger}+u_{2,4, \mathbf{k}} \gamma_{2,-\mathbf{k}}^{\dagger} \\
a_{d,-\mathbf{k}}^{\dagger} & =v_{1,1, \mathbf{k}} \gamma_{1, \mathbf{k}}+v_{1,2, \mathbf{k}} \gamma_{2, \mathbf{k}}+v_{1,3, \mathbf{k}} \gamma_{1,-\mathbf{k}}^{\dagger}+v_{1,4, \mathbf{k}} \gamma_{2,-\mathbf{k}}^{\dagger} \\
a_{f b,-\mathbf{k}}^{\dagger} & =v_{2,1, \mathbf{k}} \gamma_{1, \mathbf{k}}+v_{2,2, \mathbf{k}} \gamma_{2, \mathbf{k}}+v_{2,3, \mathbf{k}} \gamma_{1,-\mathbf{k}}^{\dagger}+v_{2,4, \mathbf{k}} \gamma_{2,-\mathbf{k}}^{\dagger}
\end{aligned}
$$

For our purposes we are only interested in expressing $a_{d, \mathbf{k}}$ and $a_{d,-\mathbf{k}}$ in terms of the $\gamma_{n, \mathbf{k}}$ operators. We already have $a_{d, \mathbf{k}}$ and to find $a_{d,-\mathbf{k}}$ it is a simply matter of Hermition conjugating the term $a_{d,-\mathbf{k}}^{\dagger}$ which gives us:

$$
a_{d,-\mathbf{k}}=\left(a_{d,-\mathbf{k}}^{\dagger}\right)^{\dagger}=v_{1,1, \mathbf{k}}^{*} \gamma_{1, \mathbf{k}}^{\dagger}+v_{1,2, \mathbf{k}}^{*} \gamma_{2, \mathbf{k}}^{\dagger}+v_{1,3, \mathbf{k}}^{*} \gamma_{1,-\mathbf{k}}+v_{1,4, \mathbf{k}}^{*} \gamma_{2,-\mathbf{k}}
$$

Since we are dealing with an expectation value in the $\gamma_{n, \mathbf{k}}$ basis we will have our expectation value be $\left\langle a_{d,-\mathbf{k}} a_{d, \mathbf{k}}\right\rangle=\left\langle\Phi_{G S}\left|a_{d,-\mathbf{k}} a_{d, \mathbf{k}}\right| \Phi_{G S}\right\rangle$ where $\gamma_{n, \mathbf{k}}\left|\Phi_{G S}\right\rangle=0$ as stated above. The expression generated from the product $a_{d,-\mathbf{k}} a_{d, \mathbf{k}}$ will have sixteen separate terms in it. Many of these terms can be safely ignored however, the reasons for which will be explained below. 
In a product as we are expecting above there will be terms of three different types. The first type will have the an annihilation $\gamma_{n, \mathbf{k}}$ operator at the end of the product such as the term $u_{1,1, \mathbf{k}} v_{1,1, \mathbf{k}} \gamma_{1, \mathbf{k}}^{\dagger} \gamma_{1, \mathbf{k}}$. All terms of this first type can be evaluated to zero since the annihilation $\gamma_{n, \mathbf{k}}$ operator at the end will be the first to act on the ground state which will give a zero. The second type will have an operator pair in the form $\gamma_{n, \mathbf{k}}^{\dagger} \gamma_{n^{\prime}, \mathbf{k}}^{\dagger}$. These terms may also be safely evaluated to zero since now the inner product $\left\langle\Phi_{G S}\left|\gamma_{n, \mathbf{k}}^{\dagger} \gamma_{n^{\prime}, \mathbf{k}}^{\dagger}\right| \Phi_{G S}\right\rangle$ can be written as $\left\langle\Phi_{G S} \mid n, \mathbf{k} ; n^{\prime}, \mathbf{k}\right\rangle$ after applying the operators. This inner product now is of course zero since the two kets are orthonormal to each other in this basis. The third type of term will have operator pairs in the form $\gamma_{n, \mathbf{k}} \gamma_{n^{\prime}, \mathbf{k}}^{\dagger}$. Because we know that $\left\{a_{\alpha}, a_{\beta}\right\}=\delta_{\alpha \beta}$ for fermion operators then we may write these terms like so $\gamma_{n, \mathbf{k}} \gamma_{n^{\prime}, \mathbf{k}}^{\dagger}=\delta_{n n^{\prime}}-\gamma_{n^{\prime}, \mathbf{k}}^{\dagger} \gamma_{n, \mathbf{k}}$. We see now in this form that the only terms that will survive are those with $n=n^{\prime}$ and thus we know only those terms with the form $\gamma_{n, \mathbf{k}} \gamma_{n, \mathbf{k}}^{\dagger}$ in the expansion will be non-zero. This leaves us with only two terms of the original sixteen which we may write now as

$$
\begin{aligned}
\left\langle a_{d,-\mathbf{k}} a_{d, \mathbf{k}}\right\rangle & =\left\langle\Phi_{G S}\left|u_{1,3, \mathbf{k}} v_{1,3, \mathbf{k}}^{*} \gamma_{1,-\mathbf{k}} \gamma_{1,-\mathbf{k}}^{\dagger}+u_{1,4, \mathbf{k}} v_{1,4, \mathbf{k}}^{*} \gamma_{2,-\mathbf{k}} \gamma_{2,-\mathbf{k}}^{\dagger}\right| \Phi_{G S}\right\rangle \\
& =u_{1,3, \mathbf{k}} v_{1,3, \mathbf{k}}^{*}+u_{1,4, \mathbf{k}} v_{1,4, \mathbf{k}}^{*}
\end{aligned}
$$

We see now that from (3.29) that we will only need components from the eigenspinors $\mathbf{w}_{3}$ and $\mathbf{w}_{4}$. Writing these vectors now so that we may express (3.29) fully we have

$$
\mathbf{w}_{3}=\frac{1}{\mathcal{N}_{3}}\left[\begin{array}{c}
e^{-i \theta}\left((\mathbf{k} \cdot \mathbf{P})^{2}-\left(E_{1, \mathbf{k}}-\varepsilon_{d}\right)\left(E_{1, \mathbf{k}}-\varepsilon_{f b}\right)\right) \\
\frac{e^{-2 i \theta}}{\mathbf{k} \cdot \mathbf{P}}\left(\left(E_{1, \mathbf{k}}^{2}-\varepsilon_{d}^{2}-\left|\Delta_{d}\right|^{2}\right)\left(E_{1, \mathbf{k}}-\varepsilon_{f b}\right)-\left(E_{1, \mathbf{k}}+\varepsilon_{d}\right)(\mathbf{k} \cdot \mathbf{P})^{2}\right) \\
e^{-i \theta} \Delta_{d}^{*}\left(\varepsilon_{f b}-E_{1, \mathbf{k}}\right) \\
\Delta_{d}^{*} \mathbf{k} \cdot \mathbf{P}
\end{array}\right]
$$

and

$$
\mathbf{w}_{4}=\frac{1}{\mathcal{N}_{4}}\left[\begin{array}{c}
e^{-i \theta}\left((\mathbf{k} \cdot \mathbf{P})^{2}-\left(E_{2, \mathbf{k}}-\varepsilon_{d}\right)\left(E_{2, \mathbf{k}}-\varepsilon_{f b}\right)\right) \\
\frac{e^{-2 i \theta}}{\mathbf{k} \cdot \mathbf{P}}\left(\left(E_{2, \mathbf{k}}^{2}-\varepsilon_{d}^{2}-\left|\Delta_{d}\right|^{2}\right)\left(E_{2, \mathbf{k}}-\varepsilon_{f b}\right)-\left(E_{2, \mathbf{k}}+\varepsilon_{d}\right)(\mathbf{k} \cdot \mathbf{P})^{2}\right) \\
e^{-i \theta} \Delta_{d}^{*}\left(\varepsilon_{f b}-E_{2, \mathbf{k}}\right) \\
\Delta_{d}^{*} \mathbf{k} \cdot \mathbf{P}
\end{array}\right]
$$

where $\mathcal{N}_{3}$ and $\mathcal{N}_{4}$ are the norms of the vectors to normalise them. These norms are 
defined as:

$$
\begin{aligned}
\mathcal{N}_{3}^{2}= & \frac{1}{(\mathbf{k} \cdot \mathbf{P})^{2}}\left(\left(E_{1, \mathbf{k}}^{2}-\varepsilon_{d}^{2}-\left|\Delta_{d}\right|^{2}\right)\left(E_{1, \mathbf{k}}-\varepsilon_{f b}\right)-\left(E_{1, \mathbf{k}}+\varepsilon_{d}\right)(\mathbf{k} \cdot \mathbf{P})^{2}\right)^{2} \\
& +\left((\mathbf{k} \cdot \mathbf{P})^{2}-\left(E_{1, \mathbf{k}}-\varepsilon_{d}\right)\left(E_{1, \mathbf{k}}-\varepsilon_{f b}\right)\right)^{2}+\left|\Delta_{d}\right|^{2}\left(\varepsilon_{f b}-E_{1, \mathbf{k}}\right)^{2}+\left|\Delta_{d}\right|^{2}(\mathbf{k} \cdot \mathbf{P})^{2}
\end{aligned}
$$

and

$$
\begin{aligned}
\mathcal{N}_{4}^{2}= & \frac{1}{(\mathbf{k} \cdot \mathbf{P})^{2}}\left(\left(E_{2, \mathbf{k}}^{2}-\varepsilon_{d}^{2}-\left|\Delta_{d}\right|^{2}\right)\left(E_{2, \mathbf{k}}-\varepsilon_{f b}\right)-\left(E_{2, \mathbf{k}}+\varepsilon_{d}\right)(\mathbf{k} \cdot \mathbf{P})^{2}\right)^{2} \\
& +\left((\mathbf{k} \cdot \mathbf{P})^{2}-\left(E_{2, \mathbf{k}}-\varepsilon_{d}\right)\left(E_{2, \mathbf{k}}-\varepsilon_{f b}\right)\right)^{2}+\left|\Delta_{d}\right|^{2}\left(\varepsilon_{f b}-E_{2, \mathbf{k}}\right)^{2}+\left|\Delta_{d}\right|^{2}(\mathbf{k} \cdot \mathbf{P})^{2}
\end{aligned}
$$

Thus we may now write our expectation value as

$$
\begin{aligned}
&\left\langle a_{d,-\mathbf{k}^{\prime}} a_{d, \mathbf{k}^{\prime}}\right\rangle=\Delta_{d}\left[\frac{\left(\varepsilon_{f b}-E_{1, \mathbf{k}}\right)\left((\mathbf{k} \cdot \mathbf{P})^{2}-\left(E_{1, \mathbf{k}}-\varepsilon_{d}\right)\left(E_{1, \mathbf{k}}-\varepsilon_{f b}\right)\right)}{\mathcal{N}_{3}^{2}}+\right. \\
&\left.\frac{\left(\varepsilon_{f b}-E_{2, \mathbf{k}}\right)\left((\mathbf{k} \cdot \mathbf{P})^{2}-\left(E_{2, \mathbf{k}}-\varepsilon_{d}\right)\left(E_{2, \mathbf{k}}-\varepsilon_{f b}\right)\right)}{\mathcal{N}_{4}^{2}}\right] \\
&=\Delta_{d} E V(\mathbf{k})
\end{aligned}
$$

where for simplicity we have absorbed the complicated expression in the square brackets into some function $E V(\mathbf{k})$ ( $E V$ standing for 'expectation value'). Finally the self consistency equation can be written, whereby we convert the sum over $\mathbf{k}$ into an integral:

$$
\Delta_{d}(\mathbf{k})=2 V_{2} \frac{\Omega}{(2 \pi)^{3}} \int d k^{\prime 3} \mathbf{k} \cdot \mathbf{k}^{\prime} \Delta_{d}\left(\mathbf{k}^{\prime}\right) E V\left(\mathbf{k}^{\prime}\right)
$$

This will the form of our self-consistency equation which we note has marked similarities to the self consistency in subsection 1.2.3 due to the $\mathbf{k} \cdot \mathbf{k}^{\prime}$ term in the integral. We will use this similarity to treat our integral (3.35) in much the same way.

\subsubsection{Treating the Self Consistency Integral}

Before we begin to treat the integral we may begin to simplify the problem by assuming, without loss of generality, that the $\mathbf{P}$ vector (which is real) points in the $z$ direction, i.e. $\mathbf{P}=P \hat{z}$. This means that we may write any $\mathbf{k} \cdot \mathbf{P}$ terms as $k P \cos \theta$ where $\theta$ is the angle from the $z$-axis to the vector $\mathbf{k}$. Another consequence of this 
choice is that we may now write the vector $\mathbf{k}$ in spherical coordinates as usual:

$$
\mathbf{k}=k\left[\begin{array}{lll}
\cos \phi \sin \theta, & \sin \phi \sin \theta, \quad \cos \theta
\end{array}\right]^{T}
$$

which allows us to express the $\mathbf{k} \cdot \mathbf{k}^{\prime}$ term as

$$
\begin{aligned}
\mathbf{k} \cdot \mathbf{k}^{\prime} & =k k^{\prime}\left(\cos \phi \cos \phi^{\prime} \sin \theta \sin \theta^{\prime}+\sin \phi \sin \phi^{\prime} \sin \theta \sin \theta^{\prime}+\cos \theta \cos \theta^{\prime}\right) \\
& =k k^{\prime}\left(\cos \left(\phi-\phi^{\prime}\right) \sin \theta \sin \theta^{\prime}+\cos \theta \cos \theta^{\prime}\right)
\end{aligned}
$$

where the last equality comes from the trigonometric identity: $\cos (A \mp B)=$ $\sin A \sin B \pm \cos A \cos B$.

As was mentioned in subsection 1.2.3 we expect three types of pairing symmetries in $3 \mathrm{D}$ that $\Delta_{d}$ can take as a $p$-wave superconductor. These were given in (1.18) and were called as the $p_{z^{-}}$and $p_{x \pm i y}$-pairing symmetries. For our uses we will make Ansätze for the form these three symmetries may take. These will be:

$$
\Delta_{d, z}(\mathbf{k})=\frac{k_{z}}{k_{F}} \Delta_{0}^{(0)}
$$

and

$$
\Delta_{d, x \pm i y}(\mathbf{k})=\frac{k_{x} \pm i k_{y}}{k_{F}} \Delta_{0}^{( \pm 1)}
$$

where $k_{F}$ is the Fermi wavevector, the $\Delta_{0}$ terms are constants and the superscripts for the $\Delta_{0}$ terms are in keeping with equation (1.18). In keeping with the changes to spherical coordinates we may now rewrite our Ansätze (3.38) and (3.39) as:

$$
\Delta_{d, z}(\mathbf{k})=\frac{k}{k_{F}} \cos \theta \Delta_{0}^{(0)}
$$

and

$$
\Delta_{d, x \pm i y}(\mathbf{k})=\frac{k}{k_{F}} \sin \theta e^{ \pm i \phi} \Delta_{0}^{( \pm 1)} .
$$

We will now attempt to reduce the complexity of the self consistency integral for all three cases for a general $E V(\mathbf{k})$. We note here that based on these current definitions our expression for $E V(\mathbf{k})$ will have no azimuthal dependence, i.e. it will not depend on the angle $\phi$.

\section{$p_{z}$ Pairing Symmetry}

For the $p_{z}$-symmetry case we substitute our expressions (3.37) and (3.40) into the integral (3.35) which has been moved to spherical coordinates via the transformation 
(1.19). The result of such operations leaves us with the expression:

$$
\begin{aligned}
\frac{k}{k_{F}} \cos \theta \Delta_{0}^{(0)}=2 \frac{k}{k_{F}} \Delta_{0}^{(0)} & V_{2} \\
& \frac{\Omega}{(2 \pi)^{3}} \int d k^{\prime} \int_{0}^{\pi} d \theta^{\prime} \int_{0}^{2 \pi} d \phi^{\prime} k^{\prime 4} \sin \theta^{\prime} \cos \theta^{\prime} \\
& \times\left(\cos \left(\phi-\phi^{\prime}\right) \sin \theta \sin \theta^{\prime}+\cos \theta \cos \theta^{\prime}\right) E V\left(k^{\prime}, \theta^{\prime}\right)
\end{aligned}
$$

Immediately we see cancellations on the right and left hand side of (3.42) and we also recognise that the integral $\int_{0}^{2 \pi} \cos \left(\phi-\phi^{\prime}\right) d \phi^{\prime}=0$ due to the periodicity of the sin function. Thus we may reduce (3.42) to:

$$
\cos \theta=2 V_{2} \cos \theta \frac{2 \pi \Omega}{(2 \pi)^{3}} \int d k^{\prime} \int_{0}^{\pi} d \theta^{\prime} k^{\prime 4} \sin \theta^{\prime} \cos ^{2} \theta^{\prime} E V\left(k^{\prime}, \theta^{\prime}\right)
$$

which can be easily simplified to:

$$
1=\frac{2 V_{2} \Omega}{(2 \pi)^{2}} \int d k^{\prime} \int_{0}^{\pi} d \theta^{\prime} k^{4} \sin \theta^{\prime} \cos ^{2} \theta^{\prime} E V\left(k^{\prime}, \theta^{\prime}\right)
$$

At this stage we can do no more without using the actual form of $E V(k, \theta)$. However, we can still simplify the integral even without knowing $E V(k, \theta)$ at this stage. Referring back to subsection 1.2.1 we will also use the fact that we assume the interaction strength is non-vanishing only in a thin shell about the Fermi energy $\varepsilon_{F}$. Thus we restrict the $k$ integral such that the energies lie within the range $\left(\varepsilon_{F}-\hbar \omega_{D}, \varepsilon_{F}+\hbar \omega_{D}\right)$ where $\omega_{D}$ is the Debye frequency. This can be written then as a constraint for the wavevectors that satisfy

$$
\varepsilon_{F}-\hbar \omega_{D}<\frac{\hbar^{2}}{2 m_{d \uparrow}} k^{2}+\varepsilon_{d, 0}<\varepsilon_{F}+\hbar \omega_{D}
$$

since $\varepsilon_{d}$ is the only energy band that depends on wavevector in the system.

Since the form of $E V(k, \theta)$ is quite complicated we introduce the following approximation which will serve to simplify (3.44). As was suggested via private communications with Moghaddam et al. [91] we move to linearize the dispersion $\varepsilon_{d, \mathbf{k}}$ about the Fermi energy. Thus we may write

$$
\varepsilon_{d} \approx \hbar v_{F}\left(k-k_{F}\right)
$$

where $v_{F}=\left.\frac{1}{\hbar} \frac{\partial \varepsilon_{d}}{\partial k}\right|_{k=k_{F}}$ is the Fermi-velocity (the speed of the electrons in the $d$-band at the Fermi-level) and $k_{F}$ is the Fermi-wavevector. This is true for small values of $k-k_{F}$ and so now we may define $k_{D}=\frac{\omega_{D}}{v_{F}}$ such that the energy condition (3.45) now restricts the wavevectors to the range $\left(k_{F}-k_{D}, k_{F}+k_{D}\right)$ where based on our 
assumptions now we take $k_{F} \gg k_{D}$. Now our integral (3.44) is written

$$
1=\frac{2 V_{2} \Omega}{(2 \pi)^{2}} \int_{k_{F}-k_{D}}^{k_{F}+k_{D}} d k^{\prime} \int_{0}^{\pi} d \theta^{\prime} k^{\prime 4} \sin \theta^{\prime} \cos ^{2} \theta^{\prime} E V\left(k^{\prime}, \cos \theta^{\prime}\right)
$$

For the sake of simplicity we make two substitutions, the first being $u=\cos \theta^{\prime}$ and the second being $q=k^{\prime}-k_{F}$. This transforms the integral to

$$
1=\frac{2 V_{2} \Omega}{(2 \pi)^{2}} \int_{-k_{D}}^{+k_{D}} d q \int_{-1}^{1} d u\left(q+k_{F}\right)^{4} u^{2} E V(q, u)
$$

Using that $k_{F} \gg k_{D}$ we see that of course $q \ll k_{F}$ as well since the greatest value $q$ attains is $k_{D}$. Now we may replace all factors of $q+k_{F}$ (corresponding to $k^{\prime}$ terms) with $k_{F}$. This results in the integral

$$
1=\frac{2 V_{2} k_{F}^{4} \Omega}{(2 \pi)^{2}} \int_{-k_{D}}^{+k_{D}} d q \int_{-1}^{1} d u u^{2} E V(q, u)
$$

This is the simplest we can make this integral without using the specific form of $E V(q, u)$. We shall repeat this for the $p_{x \pm i y}$ case after which we may look to incorporating $E V(q, u)$ into our considerations.

\section{$p_{x \pm i y}$ Pairing Symmetry}

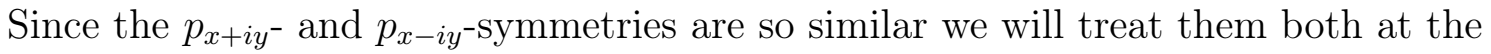
same time. We will see that the choice of either one does not impact the integral in any significant way. Just as we have done for the $p_{z}$-symmetry case we will end up with a very similar integral to deal with:

$$
\begin{array}{r}
\frac{k}{k_{F}} \sin \theta e^{ \pm i \phi} \Delta_{0}^{( \pm 1)}=2 \frac{k}{k_{F}} \Delta_{0}^{( \pm 1)} V_{2} \frac{\Omega}{(2 \pi)^{3}} \int d k^{\prime} \int_{0}^{\pi} d \theta^{\prime} \int_{0}^{2 \pi} d \phi^{\prime} k^{\prime 4} \sin ^{2} \theta^{\prime} e^{ \pm i \phi^{\prime}} \\
\times\left(\cos \left(\phi-\phi^{\prime}\right) \sin \theta \sin \theta^{\prime}+\cos \theta \cos \theta^{\prime}\right) E V\left(k^{\prime}, \theta^{\prime}\right)
\end{array}
$$

Once again we see there are immediate cancellations to be made. The integral in $\phi^{\prime}$ however is now slightly more complicated in this case. The solution however is not complicated and beautifully balances out the left hand side, just as we have seen before for the other symmetry. Firstly we recognise the term that will only have the contribution $\int_{0}^{2 \pi} e^{ \pm i \phi^{\prime}} d \phi^{\prime}$ will be zero. Secondly we find that the integral $\int_{0}^{2 \pi} e^{ \pm i \phi^{\prime}} \cos \left(\phi-\phi^{\prime}\right) d \phi^{\prime}$ has the analytic solution:

$$
\int_{0}^{2 \pi} e^{ \pm i \phi^{\prime}} \cos \left(\phi-\phi^{\prime}\right) d \phi^{\prime}=\pi e^{ \pm i \phi}
$$




\subsection{RESULTS}

Using this solution we find that (3.50) reduces to:

$$
\sin \theta e^{ \pm i \phi}=2 V_{2} \sin \theta e^{ \pm i \phi} \frac{\pi \Omega}{(2 \pi)^{3}} \int d k^{\prime} \int_{0}^{\pi} d \theta^{\prime} k^{\prime 4} \sin ^{3} \theta^{\prime} E V\left(k^{\prime}, \theta^{\prime}\right)
$$

which can be easily simplified to:

$$
1=\frac{V_{2} \Omega}{(2 \pi)^{2}} \int d k^{\prime} \int_{0}^{\pi} d \theta^{\prime} k^{4} \sin ^{3} \theta^{\prime} E V\left(k^{\prime}, \theta^{\prime}\right)
$$

From here the same argument as for the $p_{z}$-symmetry applies and we can easily obtain:

$$
1=\frac{V_{2} k_{F}^{4} \Omega}{(2 \pi)^{2}} \int_{-k_{D}}^{+k_{D}} d q \int_{-1}^{1} d u\left(1-u^{2}\right) E V(q, u) .
$$

\subsection{Results}

Due to the overall complexity of $E V(q, u)$ it is difficult to extract analytic expressions for its behaviour. As such, we move to the special case where the Fermi level is raised to the energy of the $f$ band leaving $\varepsilon_{f b}=0$. This simplifies much of the algebra and allows us to solve the integral in limiting cases of $\Delta_{0}$.

\subsubsection{Special Case When $\varepsilon_{f b}=0$}

Starting from first principles we may write our mean-field Hamiltonian for this reduced system as:

$$
H_{M . F .}(\mathbf{k})=\frac{1}{2} \sum_{\mathbf{k}} \vec{a}_{\mathbf{k}}^{\dagger}\left[\begin{array}{cccc}
\hbar v_{F} q & e^{i \theta} \mathbf{k} \cdot \mathbf{P} & -\Delta_{d} & 0 \\
e^{-i \theta} \mathbf{k} \cdot \mathbf{P} & 0 & 0 & 0 \\
-\Delta_{d}^{*} & 0 & -\hbar v_{F} q & e^{-i \theta} \mathbf{k} \cdot \mathbf{P} \\
0 & 0 & e^{i \theta} \mathbf{k} \cdot \mathbf{P} & 0
\end{array}\right] \vec{a}_{\mathbf{k}}
$$

where we have made the substitution (3.46). Diagonalising this as we have discussed previously gives the eigenenergies:

$$
\begin{aligned}
E_{1}(\mathbf{k})=\frac{1}{\sqrt{2}}\left(2(\mathbf{k} \cdot \mathbf{P})^{2}\right. & +\hbar^{2} v_{F}^{2} q^{2}+\left|\Delta_{d}\right|^{2} \\
& \left.+\sqrt{\hbar^{2} v_{F}^{2} q^{2}+\left|\Delta_{d}\right|^{2}} \sqrt{\hbar^{2} v_{F}^{2} q^{2}+\left|\Delta_{d}\right|^{2}+4(\mathbf{k} \cdot \mathbf{P})^{2}}\right)^{\frac{1}{2}}
\end{aligned}
$$




$$
\begin{gathered}
E_{2}(\mathbf{k})=\frac{1}{\sqrt{2}}\left(2(\mathbf{k} \cdot \mathbf{P})^{2}+\hbar^{2} v_{F}^{2} q^{2}+\left|\Delta_{d}\right|^{2}\right. \\
\left.-\sqrt{\hbar^{2} v_{F}^{2} q^{2}+\left|\Delta_{d}\right|^{2}} \sqrt{\hbar^{2} v_{F}^{2} q^{2}+\left|\Delta_{d}\right|^{2}+4(\mathbf{k} \cdot \mathbf{P})^{2}}\right)^{\frac{1}{2}} \\
E_{3}(\mathbf{k})=-E_{1}(\mathbf{k}) \\
E_{4}(\mathbf{k})=-E_{2}(\mathbf{k})
\end{gathered}
$$

and allows us to write the expectation value in the same form as (3.34), i.e. $\left\langle a_{d,-\mathbf{k}^{\prime}} a_{d, \mathbf{k}^{\prime}}\right\rangle=$ $\Delta_{d} E V(\mathbf{k})$ with

$$
E V(\mathbf{k})=\frac{E_{1, \mathbf{k}}\left(E_{1, \mathbf{k}}^{2}-(\mathbf{k} \cdot \mathbf{P})^{2}-E_{1, \mathbf{k}} \hbar v_{F} q\right)}{\mathcal{N}_{1}^{2}}+\frac{E_{2, \mathbf{k}}\left(E_{2, \mathbf{k}}^{2}-(\mathbf{k} \cdot \mathbf{P})^{2}-E_{2, \mathbf{k}} \hbar v_{F} q\right)}{\mathcal{N}_{2}^{2}}
$$

where

$$
\begin{aligned}
& \mathcal{N}_{1}^{2}=\left(\hbar^{2} v_{F}^{2} q^{2}+\left|\Delta_{d}\right|^{2}\right) \\
& \times\left(4(\mathbf{k} \cdot \mathbf{P})^{2}+\hbar^{2} v_{F}^{2} q^{2}+\left|\Delta_{d}\right|^{2}+\sqrt{\hbar^{2} v_{F}^{2} q^{2}+\left|\Delta_{d}\right|^{2}} \sqrt{\hbar^{2} v_{F}^{2} q^{2}+\left|\Delta_{d}\right|^{2}+4(\mathbf{k} \cdot \mathbf{P})^{2}}\right) \\
& \quad-2 E_{1, \mathbf{k}} \hbar v_{F} q \sqrt{\hbar^{2} v_{F}^{2} q^{2}+\left|\Delta_{d}\right|^{2}} \sqrt{\hbar^{2} v_{F}^{2} q^{2}+\left|\Delta_{d}\right|^{2}+4(\mathbf{k} \cdot \mathbf{P})^{2}}
\end{aligned}
$$

and

$$
\begin{aligned}
& \mathcal{N}_{2}^{2}=\left(\hbar^{2} v_{F}^{2} q^{2}+\left|\Delta_{d}\right|^{2}\right) \\
& \times\left(4(\mathbf{k} \cdot \mathbf{P})^{2}+\hbar^{2} v_{F}^{2} q^{2}+\left|\Delta_{d}\right|^{2}-\sqrt{\hbar^{2} v_{F}^{2} q^{2}+\left|\Delta_{d}\right|^{2}} \sqrt{\hbar^{2} v_{F}^{2} q^{2}+\left|\Delta_{d}\right|^{2}+4(\mathbf{k} \cdot \mathbf{P})^{2}}\right) \\
& \quad+2 E_{2, \mathbf{k}} \hbar v_{F} q \sqrt{\hbar^{2} v_{F}^{2} q^{2}+\left|\Delta_{d}\right|^{2}} \sqrt{\hbar^{2} v_{F}^{2} q^{2}+\left|\Delta_{d}\right|^{2}+4(\mathbf{k} \cdot \mathbf{P})^{2}}
\end{aligned}
$$

It turns out that after some mathematical massaging of (3.59) it can be simplified down to (see A)

$$
E V(\mathbf{k})=\frac{1}{2 \sqrt{4(\mathbf{k} \cdot \mathbf{P})^{2}+\hbar^{2} v_{F}^{2} q^{2}+\left|\Delta_{d}\right|^{2}}}
$$

From here we may integrate (3.62) in the integral (3.49) and (3.54) for each of the different symmetries. 


\subsection{RESULTS}

\section{$p_{z}$ Pairing Symmetry}

Using the specific form of $\Delta_{d, z}$ we can now write the integral $I_{z}$ :

$$
I_{z}=\frac{2 V_{2} k_{F}^{4} \Omega}{(2 \pi)^{2}} \int_{-k_{D}}^{+k_{D}} d q \int_{-1}^{1} d u \frac{u^{2}}{2 \sqrt{4 k_{F}^{2} P^{2} u^{2}+\hbar^{2} v_{F}^{2} q^{2}+\left(\Delta_{0}^{(0)}\right)^{2} u^{2}}}
$$

The integral in $q$ for this expression exists and can be shown to give the result:

$$
I_{z}=\frac{2 V_{2} k_{F}^{4} \Omega}{(2 \pi)^{2} \hbar v_{F}} \int_{-1}^{1} d u u^{2} \operatorname{Arcsinh}\left(\frac{\hbar v_{F} k_{D}}{k_{F} P|u| \sqrt{4+\left(\frac{\Delta_{0}^{(0)}}{k_{F} P}\right)^{2}}}\right)
$$

Now assuming the factor $\frac{v_{F} k_{D}}{k_{F} P|u| \sqrt{4+\left(\frac{\Delta_{0}^{(0)}}{k_{F} P}\right)^{2}}}$ is small (i.e. $v_{F} k_{D}<k_{F} P$ ) then we may take the Maclaurin series of $\operatorname{Arsinh}(\mathrm{x})$, which is $\operatorname{Arcsinh}(x) \approx x$. Thus the integral becomes:

$$
I_{z}=\frac{2 V_{2} k_{F}^{4} \Omega}{(2 \pi)^{2}} \frac{k_{D}}{k_{F} P \sqrt{4+\left(\frac{\Delta_{0}^{(0)}}{k_{F} P}\right)^{2}}} \int_{-1}^{1} \frac{u^{2}}{|u|} d u
$$

This integral is elementary with $\int_{-1}^{1} \frac{u^{2}}{|u|} d u=1$. Now we find

$$
I_{z}=\frac{2 V_{2} k_{D} k_{F}^{4} \Omega}{(2 \pi)^{2} k_{F} P \sqrt{4+\left(\frac{\Delta_{0}^{(0)}}{k_{F} P}\right)^{2}}}
$$

Connecting back to the self consistency equation we will have

$$
\begin{aligned}
1 & =\frac{2 V_{2} k_{D} k_{F}^{4} \Omega}{(2 \pi)^{2} k_{F} P \sqrt{4+\left(\frac{\Delta_{0}^{(0)}}{k_{F} P}\right)^{2}}} \\
\rightarrow \Delta_{0}^{(0)} & =2 k_{F} P \sqrt{\frac{V_{2}^{2} k_{D}^{2} k_{F}^{6} \Omega^{2}}{(2 \pi)^{4} P^{2}}-1}
\end{aligned}
$$

This result is also consistent with numerically plotted values of the integral $I_{z}\left(\Delta_{0}^{(0)}\right)$ for the entire range of $\Delta_{0}^{(0)}$ as seen in figure 3.2. This result tells that the selfconsistency is solvable, but only if we have $\frac{V_{2}^{2} k_{D}^{2} k_{F}^{6} \Omega^{2}}{(2 \pi)^{4} P^{2}}>1$. We can express this condition slightly differently by defining, as we did in subsection 1.2 .3 , the effective coupling strength $\lambda$ and the density of states at the Fermi level $N_{3 D}\left(\varepsilon_{F}\right)$ as

$$
\begin{aligned}
\lambda & =\Omega V_{2} k_{F}^{2} \\
N_{3 D}\left(\varepsilon_{F}\right) & =\frac{k_{F}^{2}}{2 \pi^{2} \hbar v_{F}}
\end{aligned}
$$




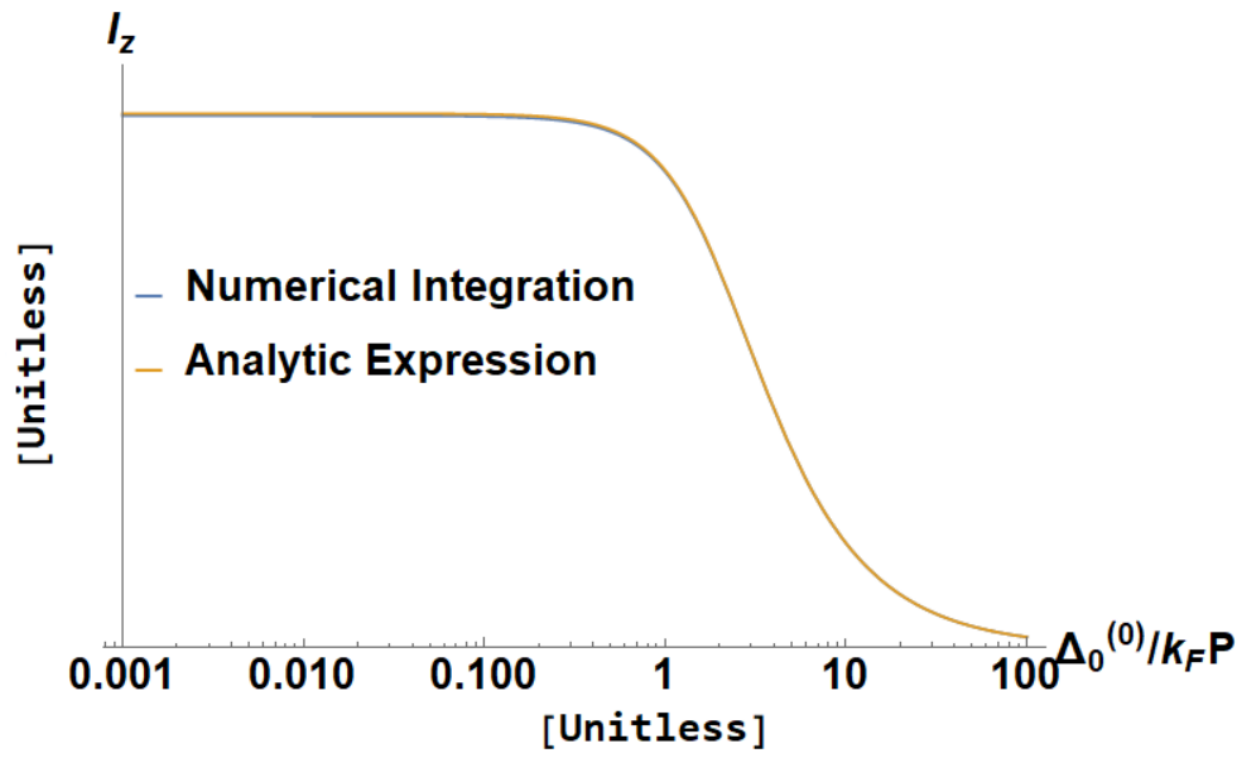

Figure 3.2: Plot of the numerically integrated integral (3.63) as a function of $\Delta_{0}^{(0)}$ (blue curve) compared to the integrated result in (3.66) (yellow curve) measured in units of $k_{F} P$. We see very good agreement between the two curves with a slight discrepancy occurring at lower values of $\Delta_{0}^{(0)}$. Note that

Now using that $k_{D}=\omega_{D} / v_{F}$ we can write the condition for solutions as

$$
\begin{aligned}
& \frac{\lambda^{2} N_{3 D}^{2}\left(\varepsilon_{F}\right) \hbar^{2} \omega_{D}^{2}}{4 k_{F}^{2} P^{2}}>1 \\
& \rightarrow \lambda>\frac{2 k_{F} P}{N_{3 D}\left(\varepsilon_{F}\right) \hbar \omega_{D}}
\end{aligned}
$$

Thus from (3.69) we see that there will be no solutions if the effective pairing potential is below this critical threshold. This is the only result where we can find an analytic expression for the whole integral, the others can only be solved for limiting values of $\Delta_{0}$ in the cases $\Delta_{0} \gg k_{F} P$ and $\Delta_{0} \ll k_{F} P$. Thus for completeness the limiting values of this integral are as follows. For $\Delta_{0}^{(0)} \gg k_{F} P$ we recover the power law:

$$
I_{z, \Delta_{0}^{(0)} \gg k_{F} P}=\frac{2 V_{2} k_{D} k_{F}^{4} \Omega}{(2 \pi)^{2} \Delta_{0}^{(0)}}=\frac{\lambda N_{3 D}\left(\varepsilon_{F}\right) \hbar \omega_{D}}{\Delta_{0}^{(0)}}
$$

Connecting this to the self-consistency condition we find that in this case we have

$$
\Delta_{0}^{(0)}=\lambda N_{3 D}\left(\varepsilon_{F}\right) \hbar \omega_{D}
$$

In this case we will find this result if the effective coupling strength is much larger than $\frac{2 k_{F} P}{N_{3 D}\left(\varepsilon_{F}\right) \hbar \omega_{D}}$ as we see from the second line in (3.67). On the other hand, for 


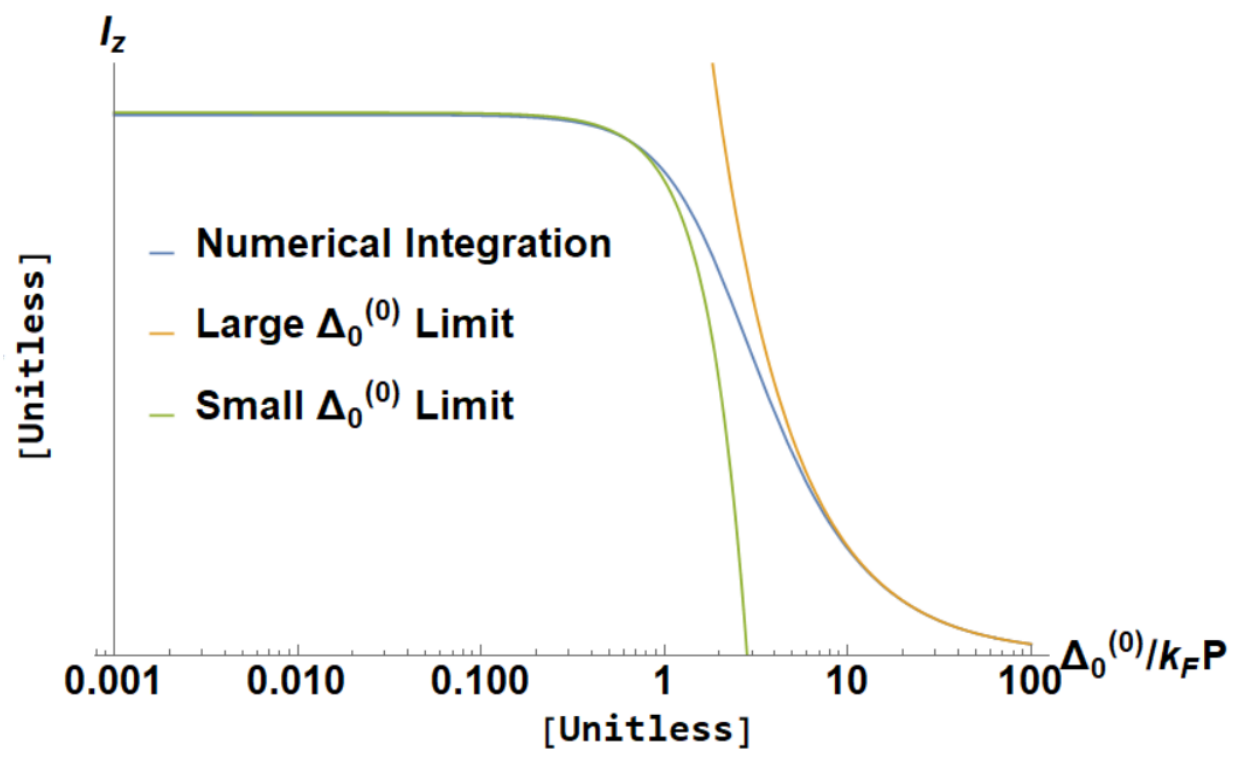

Figure 3.3: Plot of the numerically integrated integral (3.63) as a function of $\Delta_{0}^{(0)}$ (blue curve) compared to the limiting results found in (3.70) (green curve) and (3.72) (yellow curve), measured in units of $k_{F} P$. We see good agreement between the curves and the numeric result in their respective limits.

$\Delta_{0}^{(0)} \ll k_{F} P$ we can expand the square root in the first line of (3.67) to find:

$$
I_{z, \Delta_{0}^{(0)} \ll k_{F} P}=\frac{V_{2} k_{D} k_{F}^{4} \Omega}{(2 \pi)^{2} k_{F} P}\left(1-\frac{\left(\Delta_{0}^{(0)}\right)^{2}}{8 k_{F}^{2} P^{2}}\right)=\frac{\lambda N_{3 D}\left(\varepsilon_{F}\right) \hbar \omega_{D}}{2 k_{F} P}\left(1-\frac{\left(\Delta_{0}^{(0)}\right)^{2}}{8 k_{F}^{2} P^{2}}\right) .
$$

Now solving this for the self-consistency equation we find that we can express $\Delta_{0}^{(0)}$ as:

$$
\Delta_{0}^{(0)}=2 k_{F} P \sqrt{2\left(1-\frac{2 k_{F} P}{\lambda N_{3 D}\left(\varepsilon_{F}\right) \hbar \omega_{D}}\right)}
$$

where we see that the same condition for the existence of solutions as the full case (3.69) is expected from this equation, once again reinforcing the fact that in this regime the pairing is only satisfiable for large enough values of $\lambda$. These results also agree with the numerics in their respective limits as seen in figure 3.3. We note that in a real material we are likely to only be in the case where $\Delta_{0}^{(0)}$ is smaller than $k_{F} P$. This means that the low limit results are more realistic in the more likely scenarios that we encounter in the world. 


\section{$p_{x \pm i y}$ Pairing Symmetry}

Moving to the $p_{x \pm i y}$-symmetry we find we must integrate the integral $I_{x \pm i y}$ :

$$
I_{x \pm i y}=\frac{V_{2} k_{F}^{4} \Omega}{(2 \pi)^{2}} \int_{-k_{D}}^{+k_{D}} d q \int_{-1}^{1} d u \frac{1-u^{2}}{2 \sqrt{4 k_{F}^{2} P^{2} u^{2}+\hbar^{2} v_{F}^{2} q^{2}+\left(\Delta_{0}^{( \pm 1)}\right)^{2}\left(1-u^{2}\right)}}
$$

We find that the $q$ integral in (3.74) is integratable in exactly the same way as for the $p_{z}$ case. This gives us:

$$
I_{x \pm i y}=\frac{V_{2} k_{F}^{4} \Omega}{(2 \pi)^{2} \hbar v_{F}} \int_{-1}^{1} d u\left(1-u^{2}\right) \operatorname{Arcsinh}\left(\frac{\hbar v_{F} k_{D}}{\sqrt{4 k_{F}^{2} P^{2} u^{2}+\left(1-u^{2}\right)\left(\Delta_{0}^{( \pm 1)}\right)^{2}}}\right)
$$

We naively expect the same trick that we tried before to work with this integral and so we attempt the same integration. However, this does not work very well at all as it does not capture the proper lineshape and does not match the numeric result for small values of $\Delta_{0}^{( \pm 1)}$. The problem arises since we are taking only the Maclaurin series of Arcsinh in this case. If we consider the case where $\Delta_{0}^{( \pm 1)}$ is very small, then the Maclaurin series will give us the integral:

$$
\frac{V_{2} k_{F}^{4} \Omega}{(2 \pi)^{2}} \int_{-1}^{1}\left(1-u^{2}\right) \frac{k_{D}}{2 k_{F} P|u|} d u
$$

which is divergent over the integrating range due to the $\frac{1}{|u|}$ term. Even if we keep the $\left(\Delta_{0}^{(0)}\right)^{2}$ term and only discard the $\left(\Delta_{0}^{(0)}\right)^{2} u^{2}$ term in (3.75) to give

$$
\frac{V_{2} k_{F}^{4} \Omega}{(2 \pi)^{2}} \int_{-1}^{1}\left(1-u^{2}\right) \frac{k_{D}}{\sqrt{4 k_{F}^{2} P^{2} u^{2}+\left(\Delta_{0}^{( \pm 1)}\right)^{2}}} d u
$$

this integral is still divergent. This is shown by plotting the integrand of (3.75) against the integrand of (3.77) in figure 3.4. We see that the approximation is good for most of the range but diverges when $u$ is close to zero. In this case we, for small $\Delta_{0}^{( \pm 1)}$, can move to a different approximation of $\operatorname{Arcsinh}(x)$ in which $x$ becomes large. This approximation is $\operatorname{Arcsinh}(x) \approx \ln (2 x)$ and when plotted works well for very small $u$ but not for others as seen in figure 3.5. This would result in an integral of the form

$$
\frac{V_{2} k_{F}^{4} \Omega}{(2 \pi)^{2} \hbar v_{F}} \int_{-1}^{1}\left(1-u^{2}\right) \ln \left(\frac{2 \hbar v_{F} k_{D}}{\sqrt{4 k_{F}^{2} P^{2} u^{2}+\left(\Delta_{0}^{( \pm 1)}\right)^{2}}}\right) d u
$$




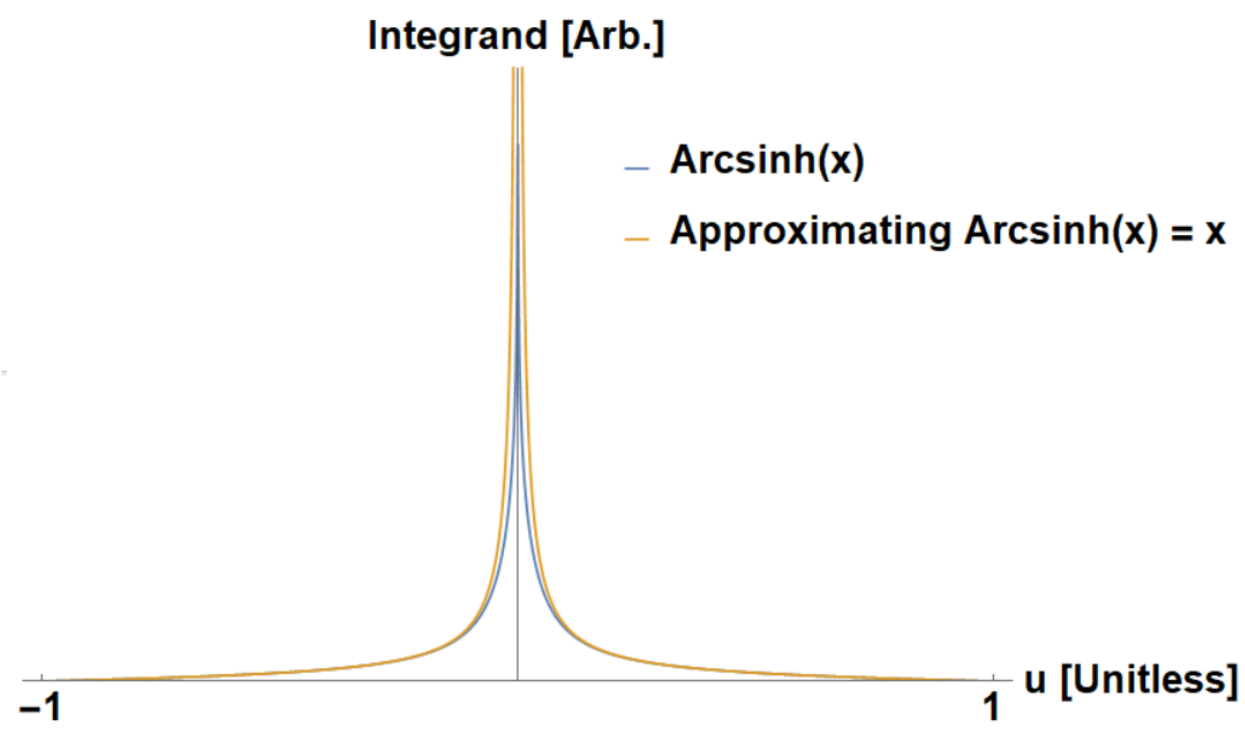

Figure 3.4: Plot of the integrand in (3.75) (blue curve) against the integrand in (3.77) (yellow curve). The approximation of $\operatorname{Arcsinh}(x) \approx x$ is good for larger values of $|u|$, but diverges as $|u|$ tends to zero.

We overcome this problem by splitting the integral over the range $\int_{-1}^{1} d u=2 \int_{0}^{1} d u$ to the two integrals $2 \int_{0}^{u_{\text {split }}} d u+2 \int_{u_{\text {split }}}^{1} d u$ where the upper integral will integrate (3.77) and the lower (3.78). We must consider where then to split the integral. If we consider the fraction $\frac{\hbar v_{F} k_{D}}{\sqrt{4 k_{F}^{2} P^{2} u^{2}+\left(\Delta_{0}^{( \pm 1)}\right)^{2}}}$ where $\Delta_{0}^{( \pm 1)}$ is small compared to the other energy scales then we see that the fraction will start to grow large when $u$ is such that $2 k_{F} P u<\hbar v_{F} k_{D}$. Thus we guess that a split when $u=\frac{\hbar v_{F} k_{D}}{2 k_{F} P}$ would yield a good approximation to the integral at small $\Delta_{0}^{( \pm 1)}$, provided of course that this quantity is less than 1. We also verify this numerically in figure 3.6 which shows excellent agreement. Thus we take in the limit for $\Delta_{0}^{( \pm 1)}$ small in comparison to the energy scales $k_{F} P$ and $\hbar v_{F} k_{D}$ the integral to be

$$
\begin{aligned}
I_{x \pm i y}= & \frac{V_{2} k_{F}^{4} \Omega}{(2 \pi)^{2} \hbar v_{F}} \int_{-1}^{1} d u\left(1-u^{2}\right) \operatorname{Arcsinh}\left(\frac{\hbar v_{F} k_{D}}{\sqrt{4 k_{F}^{2} P^{2} u^{2}+\left(1-u^{2}\right)\left(\Delta_{0}^{( \pm 1)}\right)^{2}}}\right) \\
\approx & \frac{2 V_{2} k_{F}^{4} \Omega}{(2 \pi)^{2} \hbar v_{F}}\left[\int_{0}^{\frac{\hbar v_{F} k_{D}}{2 k_{F} P}}\left(1-u^{2}\right) \ln \left(\frac{2 \hbar v_{F} k_{D}}{\sqrt{4 k_{F}^{2} P^{2} u^{2}+\left(\Delta_{0}^{( \pm 1)}\right)^{2}}}\right) d u\right. \\
& \left.+\int_{\frac{\hbar v_{F} k_{D}}{2 k_{F} P}}^{1}\left(1-u^{2}\right) \frac{\hbar v_{F} k_{D}}{\sqrt{4 k_{F}^{2} P^{2} u^{2}+\left(\Delta_{0}^{( \pm 1)}\right)^{2}}} d u\right]
\end{aligned}
$$




\section{Integrand [Arb.]}

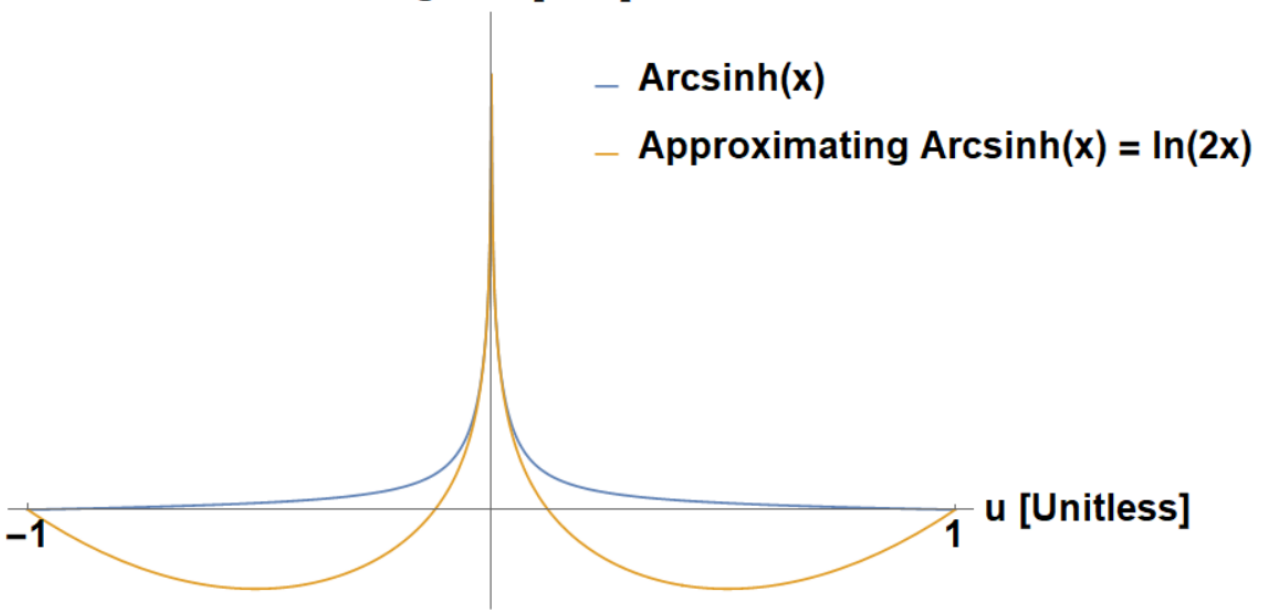

Figure 3.5: Plot of the integrand in (3.75) (blue curve) against the integrand in (3.78) (yellow curve). The approximation of $\operatorname{Arcsinh}(x) \approx \ln (2 x)$ fits well to the actual integrand for small values of $|u|$. However, we see that outside of this small window the approximation fails, quite spectacularly, to fit to the real integrand.

This expression can be analytically integrated which gives the result:

$$
\begin{aligned}
I_{x \pm i y} & =\frac{V_{2} k_{D} k_{F} \Omega}{4(2 \pi)^{2} P^{3}}\left[\frac{\hbar v_{F} k_{D}}{2} \sqrt{\left(\Delta_{0}^{( \pm 1)}\right)^{2}+\hbar^{2} v_{F}^{2} k_{D}^{2}}-k_{F} P \sqrt{\left(\Delta_{0}^{( \pm 1)}\right)^{2}+4 k_{F}^{2} P^{2}}\right. \\
& +\frac{1}{2}\left(\left(\Delta_{0}^{( \pm 1)}\right)^{2}+8 k_{F}^{2} P^{2}\right) \ln \left(\frac{2 k_{F} P+\sqrt{4 k_{F}^{2} P^{2}+\left(\Delta_{0}^{( \pm 1)}\right)^{2}}}{\hbar v_{F} k_{D}+\sqrt{\hbar^{2} v_{F}^{2} k_{D}^{2}+\left(\Delta_{0}^{( \pm 1)}\right)^{2}}}\right) \\
& +\frac{\left(\Delta_{0}^{( \pm 1)}\right)^{2}}{3}-\frac{\Delta_{0}^{( \pm 1)}}{3 \hbar v_{F} k_{D}}\left(\left(\Delta_{0}^{( \pm 1)}\right)^{2}+12 k_{F}^{2} P^{2}\right) \operatorname{Arctan}\left(\frac{\hbar v_{F} k_{D}}{\Delta_{0}^{( \pm 1)}}\right) \\
& +4 k_{F}^{2} P^{2}\left(1+\ln \left(\frac{2 \hbar v_{F} k_{D}}{\sqrt{\hbar^{2} v_{F}^{2} k_{D}^{2}+\left(\Delta_{0}^{( \pm 1)}\right)^{2}}}\right)\right) \\
& \left.-\frac{\hbar^{2} v_{F}^{2} k_{D}^{2}}{9}\left(1+3 \ln \left(\frac{2 \hbar v_{F} k_{D}}{\sqrt{\hbar^{2} v_{F}^{2} k_{D}^{2}+\left(\Delta_{0}^{( \pm 1)}\right)^{2}}}\right)\right)\right]
\end{aligned}
$$

This expression cannot be rearranged to give $\Delta_{0}^{( \pm 1)}$ by itself, however, we may still use the fact that $\Delta_{0}^{( \pm 1)}$ is smaller than the energy scales $k_{F} P$ and $\hbar v_{F} k_{D}$ to simplify this expression. If we take $\Delta_{0}^{( \pm 1)}$ to be small then we find that we may approximate $\operatorname{Arctan}\left(\frac{\hbar v_{F} k_{D}}{\Delta_{0}^{( \pm 1)}}\right) \approx \frac{\pi}{2}-\frac{\Delta_{0}^{( \pm 1)}}{\hbar v_{F} k_{D}}$ and that we do not need to consider $\left(\Delta_{0}^{( \pm 1)}\right)^{2}$ terms as the leading term in $\Delta_{0}^{( \pm 1)}$ in linear. Putting this together we find we may simplify 
(3.80) as

$$
\begin{aligned}
I_{x \pm i y} & =\frac{V_{2} k_{D} k_{F} \Omega}{4(2 \pi)^{2} P^{3}}\left[\hbar^{2} v_{F}^{2} k_{D}^{2}\left(\frac{7}{18}-\frac{1}{3} \ln 2\right)\right. \\
& \left.+2 k_{F}^{2} P^{2}\left(1+2 \ln \left(\frac{4 k_{F} P}{\hbar v_{F} k_{D}}\right)\right)-\frac{2 \pi k_{F}^{2} P^{2}}{\hbar v_{F} k_{D}} \Delta_{0}^{( \pm 1)}\right]
\end{aligned}
$$

which shows good numerical agreement with the actual integral in figure 3.6 as we expect. However, we notice that the integral (3.79) is even in $\Delta_{0}^{( \pm 1)}$ while (3.81) is not. Thus we see that $(3.81)$ can only be valid for $\Delta_{0}^{( \pm 1)}$ positive. Solving this now for $\Delta_{0}^{( \pm 1)}$ back in the self-consistency equation is now simple and yields

$$
\Delta_{0}^{( \pm 1)}=\frac{\hbar \omega_{D}}{\pi}\left[\frac{\hbar^{2} \omega_{D}^{2}}{2 k_{F}^{2} P^{2}}\left(\frac{7}{18}-\frac{1}{3} \ln 2\right)+1+2 \ln \left(\frac{4 k_{F} P}{\hbar \omega_{D}}\right)-\frac{4 k_{F} P}{\lambda N_{3 D}\left(\varepsilon_{F}\right) \hbar \omega_{D}}\right]
$$

where we have made the relevant substitutions for the effective coupling constant and density of states. From this we see that there are values of the effective coupling strength that make (3.82) negative, which make the solution invalid. This introduces another condition on the effective coupling strength for viable solutions which can be expressed as:

$$
\lambda>\frac{4 k_{F} P}{N_{3 D}\left(\varepsilon_{F}\right) \hbar \omega_{D}}\left(\frac{\hbar^{2} \omega_{D}^{2}}{2 k_{F}^{2} P^{2}}\left(\frac{7}{18}-\frac{1}{3} \ln 2\right)+1+\ln \left(\frac{4 k_{F} P}{\hbar \omega_{D}}\right)\right)^{-1}
$$

Moving onto the case when $\Delta_{0}^{( \pm 1)}$ is large, we find we may write (3.75) as

$$
I_{x \pm i y}=\frac{V_{2} k_{F}^{4} \Omega}{(2 \pi)^{2} \hbar v_{F}} \int_{-1}^{1} d u\left(1-u^{2}\right) \operatorname{Arcsinh}\left(\frac{\hbar v_{F} k_{D}}{\Delta_{0}^{( \pm 1)} \sqrt{1-u^{2}}}\right)
$$

which allows us to write the Arcsinh using its Maclaurin series since we will avoid any divergences. This gives us the integral

$$
I_{x \pm i y}=\frac{V_{2} k_{F}^{4} \Omega}{(2 \pi)^{2}} \int_{-1}^{1}\left(1-u^{2}\right) \frac{k_{D}}{\Delta_{0}^{( \pm 1)} \sqrt{1-u^{2}}} d u
$$

which is simply written as:

$$
I_{x \pm i y}=\frac{V_{2} k_{D} k_{F}^{4} \Omega}{(2 \pi)^{2} \Delta_{0}^{( \pm 1)}} \int_{-1}^{1} \sqrt{1-u^{2}} d u
$$




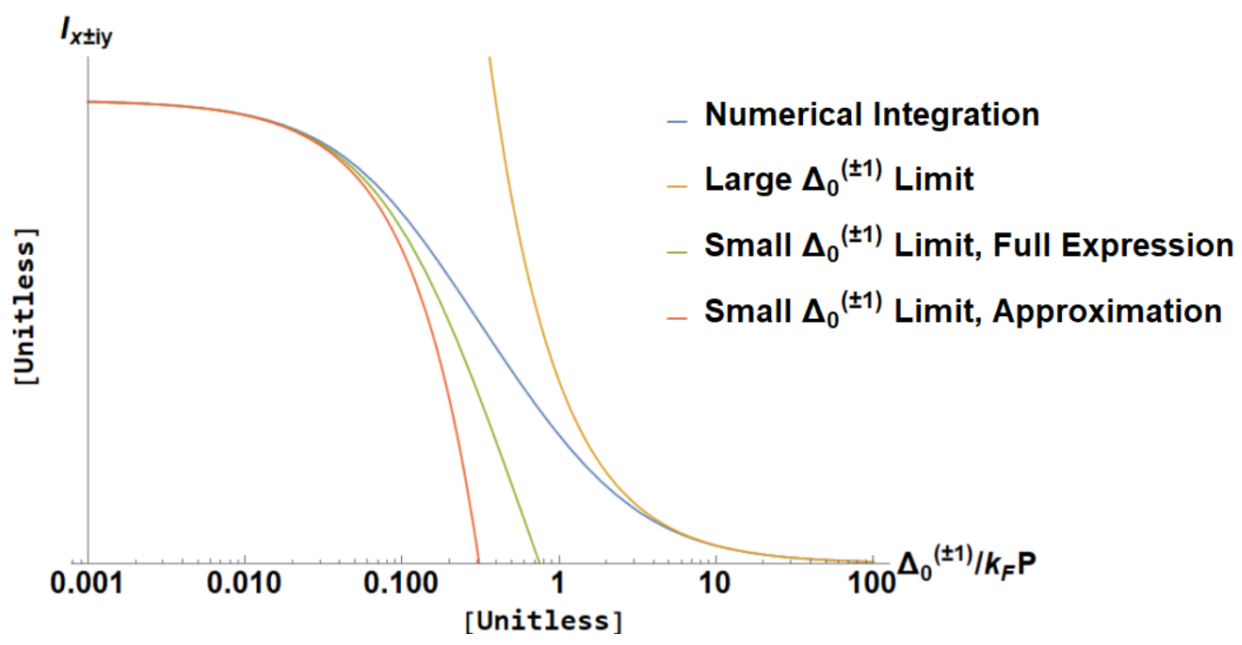

Figure 3.6: Plot of the numerically integrated integral (3.74) as a function of $\Delta_{0}^{( \pm 1)}$ (blue curve) compared to the three limiting results found in (3.87) (yellow curve), (3.81) (red curve), and (3.80) (green curve), measured in units of $k_{F} P$ The functions fit well to the numeric result in their respective limits, with the approximate function (3.81) performing well enough in the limit of small $\Delta_{0}^{( \pm 1)}$.

The integral in (3.86) has the analytic value $\int_{-1}^{1} \sqrt{1-u^{2}} d u=\frac{\pi}{2}$. Thus we find in this limit that the integral in fact is also a power law given by:

$$
I_{x \pm i y}=\frac{\pi V_{2} k_{D} k_{F}^{4} \Omega}{2(2 \pi)^{2} \Delta_{0}^{( \pm 1)}}=\frac{\pi \lambda N_{3 D}\left(\varepsilon_{F}\right) \hbar \omega_{D}}{4 \Delta_{0}^{( \pm 1)}}
$$

This can now be solved back in the self-consistency equation to give simply

$$
\Delta_{0}^{( \pm 1)}=\frac{\pi}{4} \lambda N_{3 D}\left(\varepsilon_{F}\right) \hbar \omega_{D}
$$

Numerically this once again also fits well as shown in figure 3.6. This shows that once again if the effective coupling constant is very large we find that the pairing amplitude depends linearly on the effective coupling strength, just as in the $p_{z^{-}}$ symmetry case. Both symmetries in this situation have also shown transitions into separate regimes for smaller $\lambda$ which lead to threshold values of the effective coupling strength, below which no pairing will form.

It is interesting that when the Fermi level is in this position, the pair amplitude can disappear since a naive guess would be the recovery of a result that is similar to the single band result in subsection 1.2.3. What we find instead is that there is a threshold effective coupling strength above which the superconducting state can be realised. This is due to the hybridisation between the two bands which results in an avoided crossing. The location of the Fermi level in this situation is now in 


\subsection{RESULTS}

the middle of this avoided crossing. Thus the result of a minimum effective coupling strength being required for the pairing to exist is so that the pairing can actually pair electrons across the avoided crossing.

\subsubsection{General Case With $\varepsilon_{f b} \neq 0$}

We move, in this section, to the full case where $\varepsilon_{f b}$ is nonzero and is described by (3.7). Unlike for the simplified case, at this point in time there is no known major simplification to the function $E V(\mathbf{k})$ as given in (3.34). As such we will attempt to simplify the integral by only considering the limiting cases of $\Delta_{0}$ from the onset and in fact we will find the same power law results as we did for the special case when $\Delta_{0}$ is large.

If we consider here, before moving onto the different symmetries, $\Delta_{d}$ being large, i.e. much greater than either $\varepsilon_{d, \mathbf{k}}, \varepsilon_{f b}$, or $\mathbf{k} \cdot \mathbf{P}$, then we are able to simplify large swaths of (3.34). We will begin with what will happen to $E_{1, \mathbf{k}}$ and $E_{2, \mathbf{k}}$ in such a case. We find that $E_{1, \mathbf{k}}$ will reduce to

$$
\begin{aligned}
E_{1, \mathbf{k}} & \approx \frac{1}{\sqrt{2}} \sqrt{\left|\Delta_{d}\right|^{2}+\sqrt{\left|\Delta_{d}\right|^{4}+4(\mathbf{k} \cdot \mathbf{P})^{2}\left|\Delta_{d}\right|^{2}}} \\
& \approx \frac{1}{\sqrt{2}} \sqrt{\left|\Delta_{d}\right|^{2}+\sqrt{\left|\Delta_{d}\right|^{4}}} \\
& \approx\left|\Delta_{d}\right|
\end{aligned}
$$

and, in a similar way, $E_{2, \mathbf{k}}$ reduces to

$$
\begin{aligned}
E_{2, \mathbf{k}} & \approx \frac{1}{\sqrt{2}} \sqrt{\left|\Delta_{d}\right|^{2}-\sqrt{\left|\Delta_{d}\right|^{4}+4(\mathbf{k} \cdot \mathbf{P})^{2}\left|\Delta_{d}\right|^{2}}} \\
& \approx \frac{1}{\sqrt{2}} \sqrt{\left|\Delta_{d}\right|^{2}-\sqrt{\left|\Delta_{d}\right|^{4}}} \\
& \approx 0 .
\end{aligned}
$$

Looking at the numerators of (3.34) we find the first numerator simplifies as

$$
\begin{aligned}
& \left(\varepsilon_{f b}-E_{1, \mathbf{k}}\right)\left((\mathbf{k} \cdot \mathbf{P})^{2}-\left(E_{1, \mathbf{k}}-\varepsilon_{d}\right)\left(E_{1, \mathbf{k}}-\varepsilon_{f b}\right)\right) \\
& \approx-\left|\Delta_{d}\right|\left((\mathbf{k} \cdot \mathbf{P})^{2}-\left|\Delta_{d}\right|^{2}\right) \\
& \approx\left|\Delta_{d}\right|^{3}
\end{aligned}
$$


and the second simplifies to

$$
\begin{aligned}
& \left(\varepsilon_{f b}-E_{2, \mathbf{k}}\right)\left((\mathbf{k} \cdot \mathbf{P})^{2}-\left(E_{2, \mathbf{k}}-\varepsilon_{d}\right)\left(E_{2, \mathbf{k}}-\varepsilon_{f b}\right)\right) \\
& \approx \varepsilon_{f b}\left((\mathbf{k} \cdot \mathbf{P})^{2}-\varepsilon_{f b} \varepsilon d, \mathbf{k}\right) .
\end{aligned}
$$

The norms (3.32) and (3.33) are also simplified to

$$
\begin{aligned}
\mathcal{N}_{3}^{2} & \approx \frac{1}{\mathbf{k} \cdot \mathbf{P})^{2}}\left(\left(\left|\Delta_{d}\right|^{2}-\left|\Delta_{d}\right|^{2}\right)\left|\Delta_{d}\right|-\left|\Delta_{d}\right|(\mathbf{k} \cdot \mathbf{P})^{2}\right)^{2}+\left((\mathbf{k} \cdot \mathbf{P})^{2}-\left|\Delta_{d}\right|^{2}\right)^{2} \\
& +\left|\Delta_{d}\right|^{4}+\left|\Delta_{d}\right|^{2}(\mathbf{k} \cdot \mathbf{P})^{2} \\
& \approx 2\left|\Delta_{d}\right|^{4}+2\left|\Delta_{d}\right|^{2}(\mathbf{k} \cdot \mathbf{P})^{2} \\
& \approx 2\left|\Delta_{d}\right|^{4}
\end{aligned}
$$

and

$$
\begin{aligned}
\mathcal{N}_{4}^{2} & \approx \frac{1}{(\mathbf{k} \cdot \mathbf{P})^{2}}\left(\left|\Delta_{d}\right|^{2} \varepsilon_{f b}-\varepsilon_{d, \mathbf{k}}(\mathbf{k} \cdot \mathbf{P})^{2}\right)^{2}+\left((\mathbf{k} \cdot \mathbf{P})^{2}+\varepsilon_{f b} \varepsilon_{d, \mathbf{k}}\right)^{2} \\
& +\left|\Delta_{d}\right|^{2} \varepsilon_{f b}^{2}+\left|\Delta_{d}\right|^{2}(\mathbf{k} \cdot \mathbf{P})^{2} \\
& \approx \frac{\left|\Delta_{d}\right|^{4} \varepsilon_{f b}^{2}}{(\mathbf{k} \cdot \mathbf{P})^{2}}+\left((\mathbf{k} \cdot \mathbf{P})^{2}+\varepsilon_{f b} \varepsilon_{d, \mathbf{k}}\right)^{2}+\left|\Delta_{d}\right|^{2}\left(\varepsilon_{f b}^{2}+(\mathbf{k} \cdot \mathbf{P})^{2}\right) \\
& \approx \frac{\varepsilon_{f b}^{2}}{(\mathbf{k} \cdot \mathbf{P})^{2}}\left|\Delta_{d}\right|^{4}
\end{aligned}
$$

Now we may simply write $E V(\mathbf{k})$ as:

$$
\begin{aligned}
E V(\mathbf{k}) & \approx \frac{\left|\Delta_{d}\right|^{3}}{2\left|\Delta_{d}\right|^{4}}+\frac{\varepsilon_{f b}\left((\mathbf{k} \cdot \mathbf{P})^{2}-\varepsilon_{f b} \varepsilon_{d, \mathbf{k}}\right)}{\left(\frac{\varepsilon_{f b}^{2}}{(\mathbf{k} \cdot \mathbf{P})^{2}}\left|\Delta_{d}\right|^{4}\right)} \\
& =\frac{1}{2\left|\Delta_{d}\right|^{4}}\left[\left|\Delta_{d}\right|^{3}+\frac{2 \varepsilon_{f b}(\mathbf{k} \cdot \mathbf{P})^{2}\left((\mathbf{k} \cdot \mathbf{P})^{2}-\varepsilon_{f b} \varepsilon_{d, \mathbf{k}}\right)}{\varepsilon_{f b}}\right] \\
& \approx \frac{\left|\Delta_{d}\right|^{3}}{2\left|\Delta_{d}\right|^{4}}=\frac{1}{2\left|\Delta_{d}\right|}
\end{aligned}
$$

Therefore this result holds for any form of $\left|\Delta_{d}\right|$ which is why we see such power laws appearing in this limit for the special case. With this approximation in place we may straightforwardly integrate (3.49) and (3.54). The results of which give us for 


\subsection{RESULTS}

the $p_{z}$-symmetry:

$$
\begin{aligned}
1 & =\frac{2 V_{2} k_{F}^{4} \Omega}{(2 \pi)^{2}} \int_{-k_{D}}^{+k_{D}} d q \int_{-1}^{1} d u u^{2} \operatorname{EV}(q, u) \\
& \approx \frac{2 V_{2} k_{F}^{4} \Omega}{(2 \pi)^{2}} \int_{-k_{D}}^{+k_{D}} d q \int_{-1}^{1} d u u^{2} \frac{1}{2|u| \Delta_{0}^{(0)}} \\
& =\frac{2 V_{2} k_{D} k_{F}^{4} \Omega}{(2 \pi)^{2} \Delta_{0}^{(0)}} \int_{-1}^{1} \frac{u^{2}}{|u|} d u \\
& =\frac{2 V_{2} k_{D} k_{F}^{4} \Omega}{(2 \pi)^{2} \Delta_{0}^{(0)}}
\end{aligned}
$$

which is the same result as that which we found in equation (3.70). This is not a surprising result in the limit of large $\Delta_{d}$ since in this limit $\Delta_{d}$ becomes large with respect to every energy scale. Thus we may essentially take $\varepsilon_{f b} \approx 0$, as well as the other energy scales, since $\Delta_{d}$ is the dominant term. This naturally reduces to the case where we took $\varepsilon_{f b}$ to be zero to begin with and thus is natural that we recover the same result. Therefore (3.96) has the same solution in the self-consistency equation as (3.70) which we repeat here

$$
\Delta_{0}^{(0)}=\lambda N_{3 D}\left(\varepsilon_{F}\right) \omega_{D}
$$

In the same vein we turn to the $p_{x \pm i y}$-symmetry and we find:

$$
\begin{aligned}
1 & =\frac{V_{2} k_{F}^{4} \Omega}{(2 \pi)^{2}} \int_{-k_{D}}^{+k_{D}} d q \int_{-1}^{1} d u\left(1-u^{2}\right) E V(q, u) \\
& \approx \frac{V_{2} k_{F}^{4} \Omega}{(2 \pi)^{2}} \int_{-k_{D}}^{+k_{D}} d q \int_{-1}^{1} d u\left(1-u^{2}\right) \frac{1}{2 \Delta_{0}^{( \pm 1)} \sqrt{1-u^{2}}} \\
& =\frac{V_{2} k_{D} k_{F}^{4} \Omega}{(2 \pi)^{2} k_{F} P \Delta_{0}^{( \pm 1)}} \int_{-1}^{1} \frac{1-u^{2}}{\sqrt{1-u^{2}}} d u \\
& =\frac{\pi V_{2} k_{D} k_{F}^{3} \Omega}{2(2 \pi)^{2} P \Delta_{0}^{( \pm 1)}}
\end{aligned}
$$

which, again, is the same as the result found in equation (3.87) and the same expression for $\Delta_{0}^{( \pm 1)}$ :

$$
\Delta_{0}^{( \pm 1)}=\frac{\pi}{4} \lambda N_{3 D}\left(\varepsilon_{F}\right) \omega_{D}
$$

\subsubsection{General Case; Small $\left|\Delta_{d}\right|$}

Unfortunately for the limit where $\Delta_{d}$ is small in comparison to $k_{F} P$ we have not yet found analytic expressions for the self-consistency equation. However, based on our 


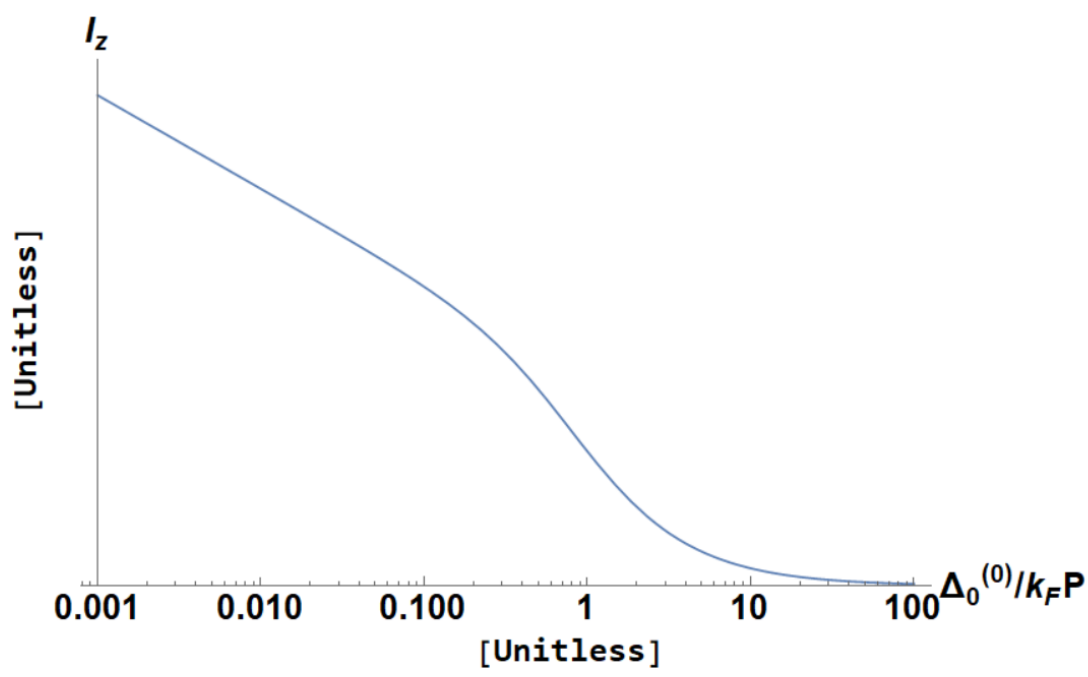

Figure 3.7: Plot of the numerically integrated integral (3.49) as a function of $\Delta_{0}^{(0)}$ measured in units of $k_{F} P$. We see that for small $\Delta_{0}^{(0)}$ the integral behaves as a negative linear line. The $x$-axis is log scaled which indicates that this behaviour can be characterised as $-\ln \left(\Delta_{0}^{(0)}\right)$ in this limit.

numerical investigations into the form of the self-consistency integrals for all three symmetry cases we have found that in this limit the integral behaves as $-\ln \Delta_{0}$. This can be seen clearly in figures 3.7 and 3.8. These figures are plotted with a $\log$ scale on the $x$-axis as $\Delta_{0}$ increases which means that any straight line we see on the graph indicates a log natural relationship. We clearly see for $\Delta_{0}$ values less than $k_{F} P$ that there is a negative straight line which is where we find the $-\ln \Delta_{0}$ behaviour. Thus in its most general we may write for the this type of integral $I$ :

$$
I=\mathcal{C}_{1}-\mathcal{C}_{2} \ln \left(\mathcal{C}_{3} \Delta_{0}\right)
$$

where $\mathcal{C}_{n}$ for $n=1,2,3$ are constants. More specifically for the $p_{z}$-symmetry case we will have

$$
I_{z}=\frac{2 V_{2} \Omega k_{C}^{4}}{(2 \pi)^{2}}\left[\mathcal{C}_{1}-\mathcal{C}_{2} \ln \left(\mathcal{C}_{3} \Delta_{0}^{(0)}\right)\right]
$$

which is easily solved for $\Delta_{0}^{(0)}$ when solving the self-consistency equation. This gives us

$$
\Delta_{0}^{(0)}=\frac{1}{\mathcal{C}_{3}} e^{\frac{\mathcal{C}_{1}}{\mathcal{C}_{2}}} \exp \left(-\frac{(2 \pi)^{2}}{2 \mathcal{C}_{2} V_{2} \Omega k_{C}^{4}}\right)
$$

which is the same form as we had in our single band case (1.20)! We can also say what units the constants $\mathcal{C}_{n}$ must have from this solution. We must have that $\left[\mathcal{C}_{1}\right]=\left[E^{-1} L^{-1}\right],\left[\mathcal{C}_{2}\right]=\left[\mathcal{C}_{1}\right]=\left[E^{-1} L^{-1}\right]$, and $\left[\mathcal{C}_{3}\right]=\left[E^{-1}\right]$. Looking at the form of (3.102) we see that the important term is the exponential containing the terms that would contribute to the effective coupling strength. Thus we see that the constants 


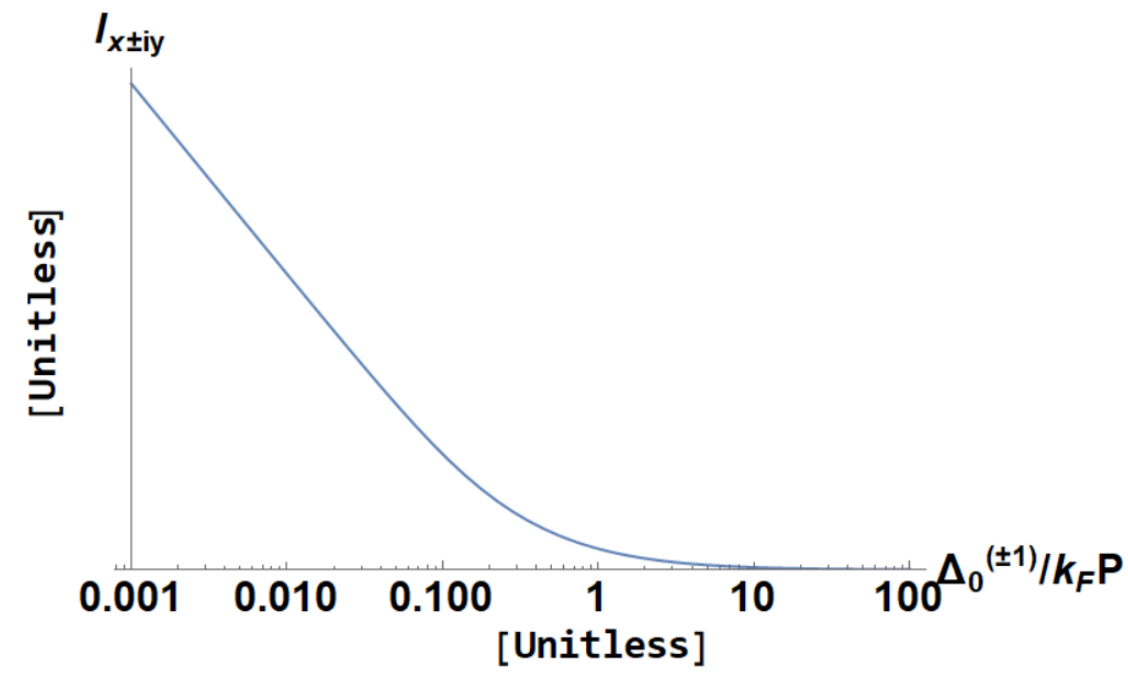

Figure 3.8: Plot of the numerically integrated integral (3.54) as a function of $\Delta_{0}^{( \pm 1)}$ measured in units of $k_{F} P$. We see again as in the $p_{z}$-symmetry case that for small $\Delta_{0}^{( \pm 1)}$ the integral behaves as a negative linear line. The $x$-axis is log scaled which indicates that this behaviour can be characterised as $-\ln \left(\Delta_{0}^{( \pm 1)}\right)$ in this limit.

$\mathcal{C}_{1}$ and $\mathcal{C}_{3}$ only contribute to a numerical factor whereas the $\mathcal{C}_{2}$ factor contains important information about the dominant exponential factor. Finding the form of this factor would allow us to categorise the pairing more effectively and could be achieved numerically by investigating the behaviour of the integral (3.49) at small values of $\Delta_{0}^{(0)}$. However, this could not be completed at the current time. In the same way we can easily see that for the $p_{x \pm i y}$ case we will have

$$
I_{x \pm i y}=\frac{V_{2} \Omega k_{C}^{4}}{(2 \pi)^{2}}\left[\mathcal{C}_{1}-\mathcal{C}_{2} \ln \left(\mathcal{C}_{3} \Delta_{0}^{( \pm 1)}\right)\right]
$$

which is solved to:

$$
\Delta_{0}^{( \pm 1)}=\frac{1}{\mathcal{C}_{3}} e^{\frac{\mathcal{C}_{1}}{\mathcal{C}_{2}}} \exp \left(-\frac{(2 \pi)^{2}}{\mathcal{C}_{2} V_{2} \Omega k_{C}^{4}}\right)
$$

While the analytic solution to the self-consistency integral could be found we do know how the integral could produce some discontinuity such that it would integrate to a $\log$ natural, i.e. a $1 / x$ dependence in either $q$ or $u$. Considering the expression for $E V$ in (3.34) it seems that the second term is the dominant term, especially at the discontinuity. It is possible to simplify this second term somewhat if we take $\left|\Delta_{d}\right|$ to be much smaller than $k_{F} P$. This simplification gives us:

$$
\begin{aligned}
E V_{\text {dominant }} & =\frac{\left(\varepsilon_{f b}-E_{2, \mathbf{k}}\right)\left(k_{F}^{2} P^{2} u^{2}-\left(E_{2, \mathbf{k}}-\varepsilon_{d}\right)\left(E_{2, \mathbf{k}}-\varepsilon_{f b}\right)\right)}{\mathcal{N}_{4}^{2}} \\
& \approx \frac{\left(\varepsilon_{f b}-E_{2, \mathbf{k}}\right) k_{F}^{2} P^{2} u^{2}}{2\left(\varepsilon_{f b}+\hbar v_{F} q\right) E_{2, \mathbf{k}}\left(\left(E_{2, \mathbf{k}}+\hbar v_{F} q\right)^{2}+k_{F}^{2} P^{2} u^{2}\right)}
\end{aligned}
$$


Now if we take the expression of $E_{2, \mathbf{k}}$ in the limit as $\left|\Delta_{d}\right|$ becomes small we find that:

$$
\begin{aligned}
E_{2, \mathbf{k}} & \approx \frac{1}{\sqrt{2}} \sqrt{\left.2 k_{F}^{2} P^{2} u^{2}+\hbar^{2} v_{F}^{2} q^{2}+\varepsilon_{f b}^{2}-\sqrt{\left(\hbar^{2} v_{F}^{2} q^{2}-\varepsilon_{f b}^{2}\right)^{2}+4 k_{F}^{2} P^{2} u^{2}\left(\hbar v_{F} q+\varepsilon_{f b}\right)^{2}}\right)} \\
& =\approx \frac{1}{\sqrt{2}} \sqrt{2 k_{F}^{2} P^{2} u^{2}+\hbar^{2} v_{F}^{2} q^{2}+\varepsilon_{f b}^{2}-\left(\hbar v_{F} q+\varepsilon_{f b}\right) \sqrt{\left(\hbar v_{F} q-\varepsilon_{f b}\right)^{2}+4 k_{F}^{2} P^{2} u^{2}}} \\
& =\frac{1}{2}\left(\sqrt{\left(\hbar v_{F} q-\varepsilon_{f b}\right)^{2}+4 k_{F}^{2} P^{2} u^{2}}-\hbar v_{F} q-\varepsilon_{f b}\right)
\end{aligned}
$$

From (3.106) it becomes clear that in this form $E_{2, \mathbf{k}}$ can go to zero. In fact it can be easily shown that it goes to zero when $q=\frac{k_{F}^{2} P^{2} u^{2}}{\hbar v_{F} \varepsilon f b}$. Numerically this is indeed where the observed divergence in the $E V$ factor is observed to be and therefore we conclude that the necessary divergence comes from the single $E_{2, \mathbf{k}}$ factor in the denominator of (3.105). If we look at $E_{2, \mathbf{k}}$ in the relevant range of integration it also seems to match the functional form $E_{2, \mathbf{k}} \propto\left|q-\frac{k_{F}^{2} P^{2} u^{2}}{\hbar v_{F} \varepsilon f b}\right|$. This is more evidence that this term is the contributing factor to the observed behaviour and also shows that if the integral only has this one divergence then we shall recover a log natural result, modified by the other $q$ dependencies in the integral. Using this knowledge will be the way forward to unravelling this integral for a possible analytic solution.

\subsection{Conclusions}

We have investigated in this chapter the possible order parameters available in this system (3.3) and based on our physical intuition we have established only the triplet pairing in the $d$ band to be significant ${ }^{2}$. We have also treated the self consistency equations for the cases where $\Delta_{d}$ is either large or small compared to the other relevant energy scales. In the situation where the Fermi level is equal to the $4 f$ band we were able to find analytic solutions in both cases. However, even though we found analytic solutions for large $\Delta_{d}$, in the general case we were not able to find analytic solutions for small $\Delta_{d}$. We have found that in all cases the gap equation will depend linearly on the effective coupling strength in the limit as $\Delta_{d}$ becomes large. More importantly is the limit when $\Delta_{d}$ is small, where we expect real systems to be. In the general situation we find that, numerically, the gap equation scales with the exponential of the effective coupling strength. This solution is not unlike the one we expect from a single band $p$-wave pairing potential as seen in subsec-

\footnotetext{
${ }^{2}$ In the absence of disorder and/or impurities
} 
tion 1.2.3. On the other hand, the order parameter for small pairing amplitudes shows anomalous behaviour when the Fermi level coincides with the $4 f$ band. In this case, for effective coupling strengths below a certain threshold, there is no solution to the self-consistency equation and therefore the superconducting state cannot be established. This occurs because the Fermi level is sitting within the middle of the avoided crossing of the mixed bands. Thus some minimum coupling strength is needed to effectively pair the electrons together across this avoided crossing. 


\section{Chapter 4}

\section{Pairing Between Mixed Bands}

Previously in chapter 3 we only considered the $d$ band interaction term in the basis of the $5 d$ and $4 f$ bands. The resultant order parameter has triplet pairing which we expect to be sensitive to disorder $[56,57]$ and may not therefore be robust enough to survive. However, what we can do is to consider how this interaction occurring in the $d$ band changes when we move to the basis of the mixed bands as seen in figure 3.1. The transformation from the basic dispersions of $\varepsilon_{d, \mathbf{k}}$ and $\varepsilon_{f b}$ to the new hybridised bands will 'mix' the interaction term which will uncover new structures for us to analyse. The motivation for this is that the mixing of the interaction term will introduce new pairing that may turn out to be resistant to disorder since it will allow pairing between electrons in the different hybridised bands now.

\subsection{The Effective Hamiltonian for the Mixed Bands}

We start by transforming our effective Hamiltonian (3.4) from the $f$ and $d$ band basis into the new mixed bands. These mixed bands are the result of diagonalising the normal state Hamiltonian (3.1) to find the new energy dispersions which we will call $\xi_{1, \mathbf{k}}$ and $\xi_{2, \mathbf{k}}$. Once we have found the correct diagonalisation matrix as in (3.16) we will be able to transform the interaction term into the new basis through some operator algebra. By moving to the mixed bands' basis we will transform from the operators $a_{d, \mathbf{k}}$ and $a_{f b, \mathbf{k}}$ to the new operators $c_{1, \mathbf{k}}$ and $c_{2, \mathbf{k}}$. The new band indices for the $c$ operators are 1 and 2, corresponding to the new mixed bands $\xi_{1, \mathbf{k}}$ and $\xi_{2, \mathbf{k}}$.

Firstly the $2 \times 2$ normal state Hamiltonian (3.1) must be diagonalised. This is a straightforward matter, however, care must still be taken since the off-diagonal terms $\left(e^{i \theta} \mathbf{k} \cdot \mathbf{P}\right)$ are not even in $\mathbf{k}$. Since from our definitions of the matrix structure for the mean-field effective Hamiltonian (3.7) we will have the matrix structure for 
the normal state Hamiltonian to be

$$
H_{N}(\mathbf{k})=\sum_{\mathbf{k}}\left[\begin{array}{ll}
a_{d, \mathbf{k}}^{\dagger} & a_{f b, \mathbf{k}}^{\dagger}
\end{array}\right]\left[\begin{array}{cc}
\varepsilon_{d, \mathbf{k}} & e^{i \theta} \mathbf{k} \cdot \mathbf{P} \\
e^{-i \theta} \mathbf{k} \cdot \mathbf{P} & \varepsilon_{f b}
\end{array}\right]\left[\begin{array}{c}
a_{d, \mathbf{k}} \\
a_{f b, \mathbf{k}}
\end{array}\right]
$$

In the same vein now as (3.16) and (3.17) we can expect to find some diagonalisation matrix $U$ to transform us into the $c_{n \mathbf{k}}$ operator basis. Diagonalising (4.1) is a straightforward exercise and we find the new hybridised bands can now be explicitly expressed as

$$
\xi_{1, \mathbf{k}}=\frac{1}{2}\left(\varepsilon_{d, \mathbf{k}}+\varepsilon_{f b}+E_{\mathbf{k}}\right)
$$

and

$$
\xi_{2, \mathbf{k}}=\frac{1}{2}\left(\varepsilon_{d, \mathbf{k}}+\varepsilon_{f b}-E_{\mathbf{k}}\right) .
$$

From the same diagonalisation we may also write the resultant $U$ as:

$$
\left[\begin{array}{c}
a_{d, \mathbf{k}} \\
a_{f b, \mathbf{k}}
\end{array}\right]=\left[\begin{array}{cc}
\cos \alpha(\mathbf{k}) & -e^{i \theta} \sin \alpha(\mathbf{k}) \operatorname{sign}(\mathbf{k} \cdot \mathbf{P}) \\
e^{-i \theta} \sin \alpha(\mathbf{k}) \operatorname{sign}(\mathbf{k} \cdot \mathbf{P}) & \cos \alpha(\mathbf{k})
\end{array}\right]\left[\begin{array}{c}
c_{1, \mathbf{k}} \\
c_{2, \mathbf{k}}
\end{array}\right] .
$$

where $\sin \alpha(\mathbf{k})$ and $\cos \alpha(\mathbf{k})$ have the forms:

$$
\begin{aligned}
& \cos \alpha(\mathbf{k})=\frac{1}{\sqrt{2}} \sqrt{1+\frac{\varepsilon_{d, \mathbf{k}}-\varepsilon_{f b}}{E_{\mathbf{k}}}} \\
& \sin \alpha(\mathbf{k})=\frac{1}{\sqrt{2}} \sqrt{1-\frac{\varepsilon_{d, \mathbf{k}}-\varepsilon_{f b}}{E_{\mathbf{k}}}}
\end{aligned}
$$

with $E_{\mathbf{k}}=\sqrt{4(\mathbf{k} \cdot \mathbf{P})^{2}+\left(\varepsilon_{d, \mathbf{k}}-\varepsilon_{f b, \mathbf{k}}\right)^{2}}$. The forms of $\sin \alpha(\mathbf{k})$ and $\cos \alpha(\mathbf{k})$ are similar to those in (1.6) and (1.16) as we expect. The expressions in (4.4) also have added $\operatorname{sign}(\mathbf{k} \cdot \mathbf{P})$ factors since the $\mathbf{k} \cdot \mathbf{P}$ element is not even in $\mathbf{k}$.

Looking more closely at the forms of $\sin \alpha(\mathbf{k})$ and $\cos \alpha(\mathbf{k})$ we see that both functions will be even in $\mathbf{k}$ since $\varepsilon_{d, \mathbf{k}}$ and $E_{\mathbf{k}}$ are even. To characterise these functions further we will consider their behaviour at $\mathbf{k}=0$, $\mathbf{k}$ large and when $\varepsilon_{d, \mathbf{k}}$ and $\varepsilon_{f b}$ are equal. If we take $\mathbf{k}=0$, assuming that $\varepsilon_{f b}$ intersects $\varepsilon_{d, \mathbf{k}}$, then $\varepsilon_{d, \mathbf{k}}-\varepsilon_{f b}$ is negative and $E_{\mathbf{k}}=\left|\varepsilon_{d, \mathbf{k}}-\varepsilon_{f b}\right|$. Thus we see we will have $\cos \alpha(0)=0$ and $\sin \alpha(0)=1$. On the other hand if $\mathbf{k}$ is large then $\varepsilon_{d, \mathbf{k}}$, then $\varepsilon_{d, \mathbf{k}}-\varepsilon_{f b}$ is positive and $E_{\mathbf{k}} \approx\left|\varepsilon_{d, \mathbf{k}}-\varepsilon_{f b}\right|$. Therefore we will see $\cos \alpha(\mathbf{k}) \rightarrow 1$ and $\sin \alpha(\mathbf{k}) \rightarrow 0$. When $\varepsilon_{d, \mathbf{k}}$ and $\varepsilon_{f b}$ are equal we see straight away that $\cos \alpha(\mathbf{k})=\sin \alpha(\mathbf{k})=\frac{1}{\sqrt{2}}$. This behaviour is summed up in the plots of $\sin \alpha(\mathbf{k})$ and $\cos \alpha(\mathbf{k})$ in figure 4.1 .

Now that we have diagonalised the normal state Hamiltonian we can move forward and transform the effective Hamiltonian to this new basis. Using (4.4) we can express the $a_{n, \mathbf{k}}$ operators in terms of the new $c_{n, \mathbf{k}}$ operators which we may then 


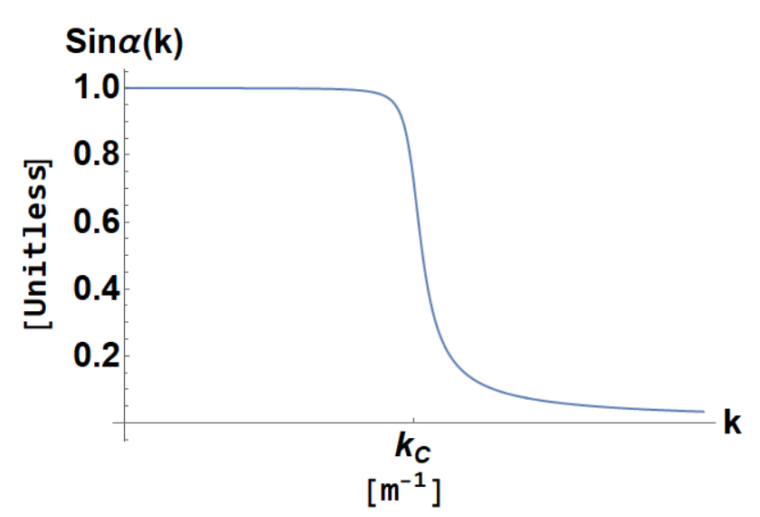

(a) $\sin \alpha(\mathbf{k})$

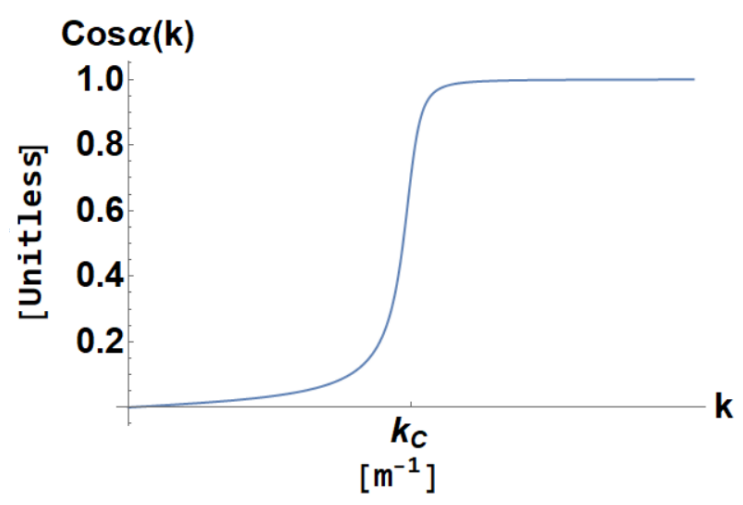

(b) $\cos \alpha(\mathbf{k})$

Figure 4.1: Plots of $\sin \alpha(\mathbf{k})$ and $\cos \alpha(\mathbf{k})$.

directly substitute into (3.4). Thus we will have the expressions:

$$
\begin{aligned}
a_{d, \mathbf{k}} & =\cos \alpha(\mathbf{k}) c_{1, \mathbf{k}}-e^{i \theta} \sin \alpha(\mathbf{k}) \operatorname{sign}(\mathbf{k} \cdot \mathbf{P}) c_{2, \mathbf{k}} \\
a_{f b, \mathbf{k}} & =e^{-i \theta} \sin \alpha(\mathbf{k}) \operatorname{sign}(\mathbf{k} \cdot \mathbf{P}) c_{1, \mathbf{k}}+\cos \alpha(\mathbf{k}) c_{2, \mathbf{k}} .
\end{aligned}
$$

We can also, through (4.7), find expressions for terms such as $a_{n,-\mathbf{k}}$ and $a_{n, \mathbf{k}}^{\dagger}$, for example:

$$
\begin{aligned}
a_{d,-\mathbf{k}} & =\cos \alpha(\mathbf{k}) c_{1,-\mathbf{k}}+e^{i \theta} \sin \alpha(\mathbf{k}) \operatorname{sign}(\mathbf{k} \cdot \mathbf{P}) c_{2,-\mathbf{k}} \\
a_{d, \mathbf{k}}^{\dagger} & =\cos \alpha(\mathbf{k}) c_{1, \mathbf{k}}^{\dagger}-e^{-i \theta} \sin \alpha(\mathbf{k}) \operatorname{sign}(\mathbf{k} \cdot \mathbf{P}) c_{2, \mathbf{k}}^{\dagger}
\end{aligned}
$$

Now we are finally in a position to transform the effective Hamiltonian which we split into two pieces. The first piece will be the diagonalised normal state $H_{N}$ and the second will be the transformed interaction $H_{\text {int }}$, i.e.

$$
\begin{aligned}
H & =H_{N}+H_{i n t} \\
& =\sum_{n, \mathbf{k}} \xi_{n, \mathbf{k}} c_{n, \mathbf{k}}^{\dagger} c_{n, \mathbf{k}}+H_{i n t}
\end{aligned}
$$

where $n=1,2$. The new interaction term, fully expanded and simplified, has the 
form

$$
\begin{aligned}
H_{i n t}= & \frac{1}{2} \sum_{\mathbf{k}, \mathbf{k}^{\prime}} V_{d d}\left(\mathbf{k}^{\prime}-\mathbf{k}\right)\left[\cos ^{2} \alpha(\mathbf{k}) \cos ^{2} \alpha\left(\mathbf{k}^{\prime}\right) c_{1, \mathbf{k}}^{\dagger} c_{1,-\mathbf{k}}^{\dagger} c_{1,-\mathbf{k}^{\prime}} c_{1, \mathbf{k}^{\prime}}\right. \\
& +\sin ^{2} \alpha(\mathbf{k}) \sin ^{2} \alpha\left(\mathbf{k}^{\prime}\right) c_{2, \mathbf{k}}^{\dagger} c_{2,-\mathbf{k}}^{\dagger} c_{2,-\mathbf{k}^{\prime}} c_{2, \mathbf{k}^{\prime}} \\
& -e^{2 i \theta} \cos ^{2} \alpha(\mathbf{k}) \sin ^{2} \alpha\left(\mathbf{k}^{\prime}\right) c_{1, \mathbf{k}}^{\dagger} c_{1,-\mathbf{k}^{\dagger}}^{\dagger} c_{2,-\mathbf{k}^{\prime}} c_{2, \mathbf{k}^{\prime}}+\text { H.c. } \\
& +\frac{1}{2} \sin 2 \alpha(\mathbf{k}) \operatorname{sign}(\mathbf{k} \cdot \mathbf{P}) \sin 2 \alpha\left(\mathbf{k}^{\prime}\right) \operatorname{sign}\left(\mathbf{k}^{\prime} \cdot \mathbf{P}\right) c_{1, \mathbf{k}}^{\dagger} c_{2,-\mathbf{k}}^{\dagger}\left[c_{2,-\mathbf{k}^{\prime}} c_{1, \mathbf{k}^{\prime}}-c_{1,-\mathbf{k}^{\prime}} c_{2, \mathbf{k}^{\prime}}\right] \\
& +e^{i \theta} \sin 2 \alpha\left(\mathbf{k}^{\prime}\right) \operatorname{sign}\left(\mathbf{k}^{\prime} \cdot \mathbf{P}\right) \cos ^{2} \alpha(\mathbf{k}) c_{1, \mathbf{k}}^{\dagger} c_{1,-\mathbf{k}}^{\dagger} c_{2,-\mathbf{k}^{\prime}} c_{1, \mathbf{k}^{\prime}}+\text { H.c. } \\
& \left.-e^{-i \theta} \sin 2 \alpha\left(\mathbf{k}^{\prime}\right) \operatorname{sign}\left(\mathbf{k}^{\prime} \cdot \mathbf{P}\right) \sin ^{2} \alpha(\mathbf{k}) c_{2, \mathbf{k}}^{\dagger} c_{2,-\mathbf{k}}^{\dagger} c_{2,-\mathbf{k}^{\prime}} c_{1, \mathbf{k}^{\prime}}+\text { H.c. }\right]
\end{aligned}
$$

where $\sin 2 \alpha(\mathbf{k})=2 \sin \alpha(\mathbf{k}) \cos \alpha(\mathbf{k})$. This expression gives us all possible pairings between electrons in either band $\xi_{1}$ or $\xi_{2}$, modified by an amplitude based on where

the electrons are in $\mathbf{k}$ space. An example of this is the third term of (4.10) which describes some hopping of electron pairs from band $\xi_{2}$ to $\xi_{1}$. While this type of interaction was excluded due to the potential's selection rules (2.15) we now have such terms occurring between the mixed bands. This interaction is modified heavily however by the prefactors $\cos ^{2} \alpha(\mathbf{k}) \sin ^{2} \alpha\left(\mathbf{k}^{\prime}\right)$ which means that this interaction only occurs when the electrons in band $\xi_{2}$ are around $\mathbf{k}=0$ and they hop to electrons with $\mathbf{k}$ large, away from the avoided crossing. Comparing with figure 3.1 we see that this is analogous to electrons scattering from low to high in the $d$ band. So while the form of $H_{\text {int }}$ has various inter and intra-band pairings they are all consistent with just interactions in the $d$ band.

\subsection{The Mean Field Hamiltonian}

Now that we have our effective Hamiltonian in the mixed band basis we can now apply the mean-field approximation to the system. In contrast to the previous meanfield Hamiltonian we will have to define more than one pair potential. These new 
pair potentials we define as

$$
\begin{aligned}
& \Delta_{1 T}(\mathbf{k})=-\sum_{\mathbf{k}^{\prime}} V_{d d}\left(\mathbf{k}-\mathbf{k}^{\prime}\right) \cos ^{2} \alpha\left(\mathbf{k}^{\prime}\right)\left\langle c_{1,-\mathbf{k}^{\prime}} c_{1, \mathbf{k}^{\prime}}\right\rangle \\
& \Delta_{2 T}(\mathbf{k})=-\sum_{\mathbf{k}^{\prime}} V_{d d}\left(\mathbf{k}-\mathbf{k}^{\prime}\right) \sin ^{2} \alpha\left(\mathbf{k}^{\prime}\right)\left\langle c_{2,-\mathbf{k}^{\prime}} c_{2, \mathbf{k}^{\prime}}\right\rangle \\
& \Delta_{1 S}(\mathbf{k})=-\sum_{\mathbf{k}^{\prime}} V_{d d}\left(\mathbf{k}-\mathbf{k}^{\prime}\right) \sin 2 \alpha\left(\mathbf{k}^{\prime}\right) \operatorname{sign}(\mathbf{k} \cdot \mathbf{P})\left\langle c_{2,-\mathbf{k}^{\prime}} c_{1, \mathbf{k}^{\prime}}\right\rangle \\
& \Delta_{2 S}(\mathbf{k})=-\sum_{\mathbf{k}^{\prime}} V_{d d}\left(\mathbf{k}-\mathbf{k}^{\prime}\right) \sin 2 \alpha\left(\mathbf{k}^{\prime}\right) \operatorname{sign}(\mathbf{k} \cdot \mathbf{P})\left\langle c_{1,-\mathbf{k}^{\prime}} c_{2, \mathbf{k}^{\prime}}\right\rangle
\end{aligned}
$$

where the $T$ and $S$ stand for triplet and singlet respectively. We label $\Delta_{1 T}$ and $\Delta_{2 T}$ as such since they have an analogous form to the triplet pair potential we introduced in chapter 3 (3.5), excepting the prefactors. The $\Delta_{1 S}$ and $\Delta_{2 S}$ are labelled as such since their definitions contain both band indices, reminiscent of singlet pairing and, as we shall see, they can be combined to form a true singlet type pairing. Using these definitions we are now able to write our mean-field interaction term which can be expressed as

$$
\begin{aligned}
H_{i n t, M F} & =-\frac{1}{2} \sum_{\mathbf{k}}\left[\cos ^{2} \alpha(\mathbf{k})\left(\Delta_{1 T, \mathbf{k}}-e^{2 i \theta} \Delta_{2 T, \mathbf{k}}+e^{i \theta} \Delta_{1 S, \mathbf{k}}\right) c_{1, \mathbf{k}}^{\dagger} c_{1,-\mathbf{k}}^{\dagger}+\right.\text { H.c. } \\
& +\sin ^{2} \alpha(\mathbf{k})\left(\Delta_{2 T, \mathbf{k}}-e^{-2 i \theta} \Delta_{1 T, \mathbf{k}}-e^{-i \theta} \Delta_{1 S, \mathbf{k}}\right) c_{2, \mathbf{k}}^{\dagger} c_{2,-\mathbf{k}}^{\dagger}+\text { H.c. } \\
& +\sin 2 \alpha(\mathbf{k}) \operatorname{sign}(\mathbf{k} \cdot \mathbf{P})\left(e^{-i \theta} \Delta_{1 T, \mathbf{k}}-e^{i \theta} \Delta_{2 T, \mathbf{k}}\right) c_{1, \mathbf{k}}^{\dagger} c_{2,-\mathbf{k}}^{\dagger}+\text { H.c. } \\
& +\frac{1}{2} \sin 2 \alpha(\mathbf{k}) \operatorname{sign}(\mathbf{k} \cdot \mathbf{P})\left(\Delta_{1 S, \mathbf{k}}-\Delta_{2 S, \mathbf{k}}\right) c_{1, \mathbf{k}}^{\dagger} c_{2,-\mathbf{k}}^{\dagger} \\
& \left.+\frac{1}{2} \sin 2 \alpha(\mathbf{k}) \operatorname{sign}(\mathbf{k} \cdot \mathbf{P}) \Delta_{1 S, \mathbf{k}}^{*}\left[c_{2,-\mathbf{k}} c_{1, \mathbf{k}}-c_{1,-\mathbf{k}} c_{2, \mathbf{k}}\right]\right]
\end{aligned}
$$

This expression (4.12) can in fact be simplified, however, it requires some rules regarding the pair potentials in (4.11) which we shall explore now. Beginning with 
$\Delta_{1 T, \mathbf{k}}$, as in (3.15), we see that we may write

$$
\begin{gathered}
\Delta_{1 T}(\mathbf{k})=-\frac{1}{2}\left[\sum_{\mathbf{k}^{\prime}} \cos ^{2} \alpha\left(\mathbf{k}^{\prime}\right) V_{d d}\left(\mathbf{k}-\mathbf{k}^{\prime}\right)\left\langle c_{1,-\mathbf{k}^{\prime}} c_{1, \mathbf{k}^{\prime}}\right\rangle\right. \\
\left.+\sum_{\mathbf{k}^{\prime} \rightarrow-\mathbf{k}^{\prime}} \cos ^{2} \alpha\left(\mathbf{k}^{\prime}\right) V_{d d}\left(\mathbf{k}-\mathbf{k}^{\prime}\right)\left\langle c_{1,-\mathbf{k}^{\prime}} c_{1, \mathbf{k}^{\prime}}\right\rangle\right] \\
=-\frac{1}{2} \sum_{\mathbf{k}^{\prime}} \cos ^{2} \alpha\left(\mathbf{k}^{\prime}\right)\left[V_{d d}\left(\mathbf{k}-\mathbf{k}^{\prime}\right)\left\langle c_{1,-\mathbf{k}^{\prime}} c_{1, \mathbf{k}^{\prime}}\right\rangle+V_{d d}\left(\mathbf{k}+\mathbf{k}^{\prime}\right)\left\langle c_{1, \mathbf{k}^{\prime}} c_{1,-\mathbf{k}^{\prime}}\right\rangle\right] \\
=-\frac{1}{2} \sum_{\mathbf{k}^{\prime}} \cos ^{2} \alpha\left(\mathbf{k}^{\prime}\right)\left(V_{d d}\left(\mathbf{k}-\mathbf{k}^{\prime}\right)-V_{d d}\left(\mathbf{k}+\mathbf{k}^{\prime}\right)\right)\left\langle c_{1,-\mathbf{k}^{\prime}} c_{1, \mathbf{k}^{\prime}}\right\rangle \\
=2 V_{2} \sum_{\mathbf{k}^{\prime}} \cos ^{2} \alpha\left(\mathbf{k}^{\prime}\right) \mathbf{k} \cdot \mathbf{k}^{\prime}\left\langle c_{1,-\mathbf{k}^{\prime}} c_{1, \mathbf{k}^{\prime}}\right\rangle .
\end{gathered}
$$

In a similar fashion we may write too

$$
\Delta_{2 T}(\mathbf{k})=2 V_{2} \sum_{\mathbf{k}^{\prime}} \cos ^{2} \alpha\left(\mathbf{k}^{\prime}\right) \mathbf{k} \cdot \mathbf{k}^{\prime}\left\langle c_{2,-\mathbf{k}^{\prime}} c_{2, \mathbf{k}^{\prime}}\right\rangle
$$

Thus we see as a natural consequence that both $\Delta_{1 T, \mathbf{k}}$ and $\Delta_{2 T, \mathbf{k}}$ must be odd in $\mathbf{k}$, i.e.

$$
\begin{aligned}
& \Delta_{1 T}\left(-\mathbf{k}^{\prime}\right)=-\Delta_{1 T}\left(\mathbf{k}^{\prime}\right) \\
& \Delta_{2 T}\left(-\mathbf{k}^{\prime}\right)=-\Delta_{2 T}\left(\mathbf{k}^{\prime}\right)
\end{aligned}
$$

Considering now $\Delta_{1 S, \mathbf{k}}$ we may repeat the same process to find:

$$
\begin{gathered}
\Delta_{1 S}(\mathbf{k})=-\sum_{\mathbf{k}^{\prime}} \operatorname{sign}\left(\mathbf{k}^{\prime} \cdot \mathbf{P}\right) \sin 2 \alpha\left(\mathbf{k}^{\prime}\right) V_{d d}\left(\mathbf{k}-\mathbf{k}^{\prime}\right)\left\langle c_{2,-\mathbf{k}^{\prime}} c_{1, \mathbf{k}^{\prime}}\right\rangle \\
=-\frac{1}{2} \sum_{\mathbf{k}^{\prime}} \operatorname{sign}\left(\mathbf{k}^{\prime} \cdot \mathbf{P}\right) \sin 2 \alpha\left(\mathbf{k}^{\prime}\right)\left[V_{d d}\left(\mathbf{k}-\mathbf{k}^{\prime}\right)\left\langle c_{2,-\mathbf{k}} c_{1, \mathbf{k}^{\prime}}\right\rangle-V_{d d}\left(\mathbf{k}+\mathbf{k}^{\prime}\right)\left\langle c_{2, \mathbf{k}^{\prime}} c_{1,-\mathbf{k}^{\prime}}\right\rangle\right] \\
=-\frac{1}{2} \sum_{\mathbf{k}^{\prime}} \operatorname{sign}\left(\mathbf{k}^{\prime} \cdot \mathbf{P}\right) \sin 2 \alpha\left(\mathbf{k}^{\prime}\right)\left[V_{d d}\left(\mathbf{k}-\mathbf{k}^{\prime}\right)\left\langle c_{2,-\mathbf{k}^{\prime}} c_{1, \mathbf{k}^{\prime}}\right\rangle+V_{d d}\left(\mathbf{k}+\mathbf{k}^{\prime}\right)\left\langle c_{1,-\mathbf{k}^{\prime}} c_{2, \mathbf{k}^{\prime}}\right\rangle\right] .
\end{gathered}
$$

Similarly for $\Delta_{2 S, \mathbf{k}}$ we find

$\Delta_{2 S}(\mathbf{k})=-\frac{1}{2} \sum_{\mathbf{k}^{\prime}} \operatorname{sign}\left(\mathbf{k}^{\prime} \cdot \mathbf{P}\right) \sin 2 \alpha\left(\mathbf{k}^{\prime}\right)\left[V_{d d}\left(\mathbf{k}-\mathbf{k}^{\prime}\right)\left\langle c_{1,-\mathbf{k}^{\prime}} c_{2, \mathbf{k}^{\prime}}\right\rangle+V_{d d}\left(\mathbf{k}+\mathbf{k}^{\prime}\right)\left\langle c_{2,-\mathbf{k}^{\prime}} c_{1, \mathbf{k}^{\prime}}\right\rangle\right]$

Now if we were to consider taking $\Delta_{2 S}(-\mathbf{k})$ we see that we will find the $V_{d d}\left(\mathbf{k}-\mathbf{k}^{\prime}\right)$ term change to $V_{d d}\left(-\mathbf{k}-\mathbf{k}^{\prime}\right)$, which is the same as $V_{d d}\left(\mathbf{k}+\mathbf{k}^{\prime}\right)$ since $V_{d d}\left(\mathbf{k}-\mathbf{k}^{\prime}\right)=$ $V_{0}\left|\mathbf{k}-\mathbf{k}^{\prime}\right|$. Similarly we see that the $V_{d d}\left(\mathbf{k}+\mathbf{k}^{\prime}\right)$ term change to $V_{d d}\left(-\mathbf{k}+\mathbf{k}^{\prime}\right)$, which 
is the same as $V_{d d}\left(\mathbf{k}-\mathbf{k}^{\prime}\right)$. Thus by comparing terms between (4.16) and (4.17) we find that in fact we will have the following rule linking $\Delta_{1 S, \mathbf{k}}$ and $\Delta_{2 S, \mathbf{k}}$ :

$$
\Delta_{2 S}(-\mathbf{k})=\Delta_{1 S}(\mathbf{k})
$$

Using these rules (4.15) and (4.18) we may now simplify the mean-field interaction term (4.12). We achieve this paradoxically by generating more terms in the expression. Examples of the manipulations we do to add terms we show below. Take the term $\frac{1}{2} \sum_{\mathbf{k}} \sin 2 \alpha(\mathbf{k}) \operatorname{sign}(\mathbf{k} \cdot \mathbf{P}) \Delta_{1 S, \mathbf{k}} c_{1, \mathbf{k}}^{\dagger} c_{2,-\mathbf{k}}^{\dagger}$ to start with. We may split this term into two as we have seen before for other such terms:

$$
\begin{gathered}
\frac{1}{4}\left[\sum_{\mathbf{k}} \sin 2 \alpha(\mathbf{k}) \operatorname{sign}(\mathbf{k} \cdot \mathbf{P}) \Delta_{1 S, \mathbf{k}} c_{1, \mathbf{k}}^{\dagger} c_{2,-\mathbf{k}}^{\dagger}+\sum_{\mathbf{k} \rightarrow \mathbf{k}} \sin 2 \alpha(\mathbf{k}) \operatorname{sign}(\mathbf{k} \cdot \mathbf{P}) \Delta_{1 S, \mathbf{k}} c_{1, \mathbf{k}}^{\dagger} c_{2,-\mathbf{k}}^{\dagger}\right] \\
=\frac{1}{4} \sum_{\mathbf{k}} \operatorname{sign}(\mathbf{k} \cdot \mathbf{P}) \sin 2 \alpha(\mathbf{k})\left[\Delta_{1 S, \mathbf{k}}\left\langle c_{1, \mathbf{k}}^{\dagger} c_{2,-\mathbf{k}}^{\dagger}\right\rangle-\Delta_{1 S,-\mathbf{k}}\left\langle c_{1,-\mathbf{k}}^{\dagger} c_{2, \mathbf{k}}^{\dagger}\right\rangle\right] \\
=\frac{1}{4} \sum_{\mathbf{k}} \operatorname{sign}(\mathbf{k} \cdot \mathbf{P}) \sin 2 \alpha(\mathbf{k})\left[\Delta_{1 S, \mathbf{k}}\left\langle c_{1, \mathbf{k}}^{\dagger} c_{2,-\mathbf{k}}^{\dagger}\right\rangle+\Delta_{2 S, \mathbf{k}}\left\langle c_{2, \mathbf{k}}^{\dagger} c_{1,-\mathbf{k}}^{\dagger}\right\rangle\right]
\end{gathered}
$$

Doing such a manipulation for terms that would appear on the off-diagonals of the resultant matrix (terms with operator pairs $c_{n, \mathbf{k}}^{\dagger} c_{n^{\prime}, \mathbf{k}}^{\dagger}$ ) we can manipulate the meanfield interaction term into such a form where we may write the matrix:

$$
H_{\text {int }}=-\frac{1}{2} \sum_{\mathbf{k}}\left[\begin{array}{ll}
c_{1, \mathbf{k}}^{\dagger} & c_{2, \mathbf{k}}^{\dagger}
\end{array}\right] \mathcal{H}_{i n t, \mathbf{k}}\left[\begin{array}{c}
c_{1,-\mathbf{k}}^{\dagger} \\
c_{2,-\mathbf{k}}^{\dagger}
\end{array}\right]
$$

where

$$
\mathcal{H}_{i n t, \mathbf{k}}=\left[\begin{array}{c}
\cos ^{2} \alpha(\mathbf{k})\left(\Delta_{1 T, \mathbf{k}}-e^{2 i \theta} \Delta_{2 T, \mathbf{k}}+\frac{1}{2} e^{i \theta}\left(\Delta_{1 S, \mathbf{k}}-\Delta_{2 S, \mathbf{k}}\right)\right) \\
-\frac{1}{2} \sin 2 \alpha(\mathbf{k}) \operatorname{sign}(\mathbf{k} \cdot \mathbf{P})\left(e^{-i \theta} \Delta_{1 T, \mathbf{k}}-e^{i \theta} \Delta_{2 T, \mathbf{k}}+\frac{1}{2}\left(\Delta_{1 S, \mathbf{k}}-\Delta_{2 S, \mathbf{k}}\right)\right) \\
\frac{1}{2} \sin 2 \alpha(\mathbf{k}) \operatorname{sign}(\mathbf{k} \cdot \mathbf{P})\left(e^{-i \theta} \Delta_{1 T, \mathbf{k}}-e^{i \theta} \Delta_{2 T, \mathbf{k}}+\frac{1}{2}\left(\Delta_{1 S, \mathbf{k}}-\Delta_{2 S, \mathbf{k}}\right)\right) \\
\sin ^{2} \alpha(\mathbf{k})\left(\Delta_{2 T, \mathbf{k}}-e^{-2 i \theta} \Delta_{1 T, \mathbf{k}}-\frac{1}{2} e^{-i \theta}\left(\Delta_{1 S, \mathbf{k}}-\Delta_{2 S, \mathbf{k}}\right)\right)
\end{array}\right]
$$

Now that we have $\mathcal{H}_{i n t, \mathbf{k}}$ in such a form we notice that every element of the matrix contains the same pattern of pair potentials. If we now define some overall pair potential $\Delta_{\mathbf{k}}$ as

$$
\Delta_{\mathbf{k}}=e^{-i \theta} \Delta_{1 T, \mathbf{k}}-e^{i \theta} \Delta_{2 T, \mathbf{k}}+\frac{1}{2}\left(\Delta_{1 S, \mathbf{k}}-\Delta_{2 S, \mathbf{k}}\right)
$$


then we see we may write (4.21) in a far more simple way:

$$
\mathcal{H}_{i n t, \mathbf{k}}=\left[\begin{array}{cc}
e^{i \theta} \cos ^{2} \alpha(\mathbf{k}) \Delta_{\mathbf{k}} & \frac{1}{2} \sin 2 \alpha(\mathbf{k}) \operatorname{sign}(\mathbf{k} \cdot \mathbf{P}) \Delta_{\mathbf{k}} \\
-\frac{1}{2} \sin 2 \alpha(\mathbf{k}) \operatorname{sign}(\mathbf{k} \cdot \mathbf{P}) \Delta_{\mathbf{k}} & -e^{-i \theta} \sin ^{2} \alpha(\mathbf{k}) \Delta_{\mathbf{k}}
\end{array}\right]
$$

Integrating this result into the full effective mean-field Hamiltonian allows us to finally write the full result in matrix form. Using the notation $\vec{c}_{\mathbf{k}}^{\dagger}=\left[c_{1, \mathbf{k}}^{\dagger}, c_{2, \mathbf{k}}^{\dagger}, c_{1,-\mathbf{k}}, c_{2,-\mathbf{k}}\right]$ we have

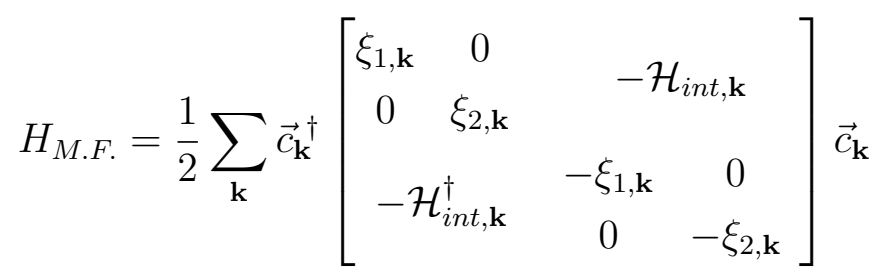

\subsubsection{Emergence of Even Parity Pairing}

Looking more closely at the overall pair potential $\Delta_{\mathbf{k}}$ we see that the two individual pair potentials $\Delta_{1 S, \mathbf{k}}$ and $\Delta_{2 S, \mathbf{k}}$ are combined in an interesting way. If we were to call this term $\Delta_{S T, \mathbf{k}}$ in the following way:

$$
\Delta_{S T, \mathbf{k}}=\frac{1}{2}\left(\Delta_{1 S, \mathbf{k}}-\Delta_{2 S, \mathbf{k}}\right)
$$

then we immediately see that we must have

$$
\Delta_{S T}(-\mathbf{k})=-\Delta_{S T}(\mathbf{k})
$$

A further consequence of this definition for $\Delta_{S, \mathbf{k}}$ is seen if we write out its definition in full:

$$
\Delta_{S T}(\mathbf{k})=-\frac{1}{2} \sum_{\mathbf{k}^{\prime}} V_{d d}\left(\mathbf{k}-\mathbf{k}^{\prime}\right) \sin 2 \alpha\left(\mathbf{k}^{\prime}\right) \operatorname{sign}\left(\mathbf{k}^{\prime} \cdot \mathbf{P}\right)\left\langle c_{2,-\mathbf{k}^{\prime}} c_{1, \mathbf{k}^{\prime}}-c_{1,-\mathbf{k}^{\prime}} c_{2, \mathbf{k}^{\prime}}\right\rangle
$$

This form is reminiscent of the singlet BCS pairing and the singlet inter-band pairing since by swapping the band indices in (4.27) would introduce a minus sign. We may 
also now write $\Delta_{S, \mathbf{k}}$ in the same for as (4.13) and (4.14):

$$
\begin{gathered}
\Delta_{S T}(\mathbf{k})=-\frac{1}{2}\left[\sum_{\mathbf{k}^{\prime}} \sin 2 \alpha\left(\mathbf{k}^{\prime}\right) V_{d d}\left(\mathbf{k}-\mathbf{k}^{\prime}\right) \operatorname{sign}(\mathbf{k} \cdot \mathbf{P})\left\langle c_{2,-\mathbf{k}^{\prime}} c_{1, \mathbf{k}^{\prime}}\right\rangle\right. \\
\left.\quad-\sum_{\mathbf{k}^{\prime} \rightarrow-\mathbf{k}^{\prime}} \sin 2 \alpha\left(\mathbf{k}^{\prime}\right) V_{d d}\left(\mathbf{k}-\mathbf{k}^{\prime}\right) \operatorname{sign}(\mathbf{k} \cdot \mathbf{P})\left\langle c_{1,-\mathbf{k}^{\prime}} c_{2, \mathbf{k}^{\prime}}\right\rangle\right] \\
=-\frac{1}{2} \sum_{\mathbf{k}^{\prime}} \sin 2 \alpha\left(\mathbf{k}^{\prime}\right) \operatorname{sign}(\mathbf{k} \cdot \mathbf{P})\left[V_{d d}\left(\mathbf{k}-\mathbf{k}^{\prime}\right)\left\langle c_{2,-\mathbf{k}^{\prime}} c_{1, \mathbf{k}^{\prime}}\right\rangle+V_{d d}\left(\mathbf{k}+\mathbf{k}^{\prime}\right)\left\langle c_{1, \mathbf{k}^{\prime}} c_{2,-\mathbf{k}^{\prime}}\right\rangle\right] \\
=-\frac{1}{2} \sum_{\mathbf{k}^{\prime}} \sin 2 \alpha\left(\mathbf{k}^{\prime}\right) \operatorname{sign}(\mathbf{k} \cdot \mathbf{P})\left(V_{d d}\left(\mathbf{k}-\mathbf{k}^{\prime}\right)-V_{d d}\left(\mathbf{k}+\mathbf{k}^{\prime}\right)\right)\left\langle c_{2,-\mathbf{k}^{\prime}} c_{1, \mathbf{k}^{\prime}}\right\rangle \\
=2 V_{2} \sum_{\mathbf{k}^{\prime}} \sin 2 \alpha\left(\mathbf{k}^{\prime}\right) \mathbf{k} \cdot \mathbf{k}^{\prime} \operatorname{sign}(\mathbf{k} \cdot \mathbf{P})\left\langle c_{2,-\mathbf{k}^{\prime}} c_{1, \mathbf{k}^{\prime}}\right\rangle .
\end{gathered}
$$

While $\Delta_{S T, \mathbf{k}}$ has itself a form reminiscent of singlet pairing we see here again that it is odd in $\mathbf{k}$ which indicates triplet behaviour. However, naturally leading on from this is the observation then that the overall pair potential $\Delta_{\mathbf{k}}$ must also be odd in $\mathbf{k}$, i.e.

$$
\Delta(-\mathbf{k})=-\Delta(\mathbf{k})
$$

Now if we consider once more $\mathcal{H}_{\text {int } \mathbf{k} \mathbf{k}}$ the diagonal elements will be odd in $\mathbf{k}$ as we expect but the off-diagonal elements must be even in $\mathbf{k}$ ! If we consider now that only $\Delta_{S T, \mathbf{k}}$ is non-zero, as we might expect in the case of adding disorder, then we find that these off-diagonal elements have singlet form due to the placement of the band indices and is even in $\mathbf{k}$. This finding we attribute to the emergence of a genuine singlet pairing between the mixed bands. With this we may make one more definition for this new singlet pairing by incorporating the prefactors on the off diagonal elements. This gives us

$$
\Delta_{\mathcal{S}}(\mathbf{k})=V_{2} \sin 2 \alpha(\mathbf{k}) \operatorname{sign}(\mathbf{k} \cdot \mathbf{P}) \sum_{\mathbf{k}^{\prime}} \sin 2 \alpha\left(\mathbf{k}^{\prime}\right) \operatorname{sign}(\mathbf{k} \cdot \mathbf{P}) \mathbf{k} \cdot \mathbf{k}^{\prime}\left\langle c_{2,-\mathbf{k}^{\prime}} c_{1, \mathbf{k}^{\prime}}\right\rangle
$$

Using this form we may also rewrite $\mathcal{H}_{\text {int }, \mathbf{k}}$ into the following form

$$
\mathcal{H}_{i n t, \mathbf{k}}=\Delta_{\mathcal{S}, \mathbf{k}}\left[\begin{array}{cc}
\frac{e^{i \theta} \operatorname{sign}(\mathbf{k} \cdot \mathbf{P})}{\tan \alpha(\mathbf{k})} & 1 \\
-1 & -e^{-i \theta} \tan \alpha(\mathbf{k}) \operatorname{sign}(\mathbf{k} \cdot \mathbf{P}) \Delta_{\mathbf{k}}
\end{array}\right]
$$

where $\tan \alpha(\mathbf{k})=\frac{\sin \alpha(\mathbf{k})}{\cos \alpha(\mathbf{k})}$. At this stage we could relate this pair potential back to the results we found in chapter 3 by expressing the $c_{n, \mathbf{k}}$ operators in terms of the original $a_{n^{\prime}, \mathbf{k}}$ operators. By inverting the transformation (4.4) and neglecting all 
terms that are not in the form $\left\langle a_{d,-\mathbf{k}} a_{d, \mathbf{k}}\right\rangle$ we find that

$$
\left\langle c_{2,-\mathbf{k}} c_{1, \mathbf{k}}\right\rangle=\frac{1}{2} e^{-i \theta} \sin 2 \alpha(\mathbf{k}) \operatorname{sign}(\mathbf{k} \cdot \mathbf{P})\left\langle a_{d,-\mathbf{k}} a_{d, \mathbf{k}}\right\rangle
$$

which allows us to express $\Delta_{\mathcal{S}, \mathbf{k}}$ as

$$
\Delta_{\mathcal{S}}(\mathbf{k})=V_{2} \sin 2 \alpha(\mathbf{k}) \operatorname{sign}(\mathbf{k} \cdot \mathbf{P}) \sum_{\mathbf{k}^{\prime}} \frac{1}{2} e^{-i \theta} \sin ^{2} 2 \alpha\left(\mathbf{k}^{\prime}\right) \mathbf{k} \cdot \mathbf{k}^{\prime}\left\langle a_{d,-\mathbf{k}^{\prime}} a_{d, \mathbf{k}^{\prime}}\right\rangle .
$$

We might have hoped that this process would have allowed us to relate $\Delta_{\mathcal{S}, \mathbf{k}}$ simply to $\Delta_{d, \mathbf{k}}$ but unfortunately this is not the case. While in theory we could continue and express the self-consistency equation in this form we choose instead to continue in the mixed band basis.

\subsection{The Self-Consistency Equation}

In order to express the self-consistency equation for $\Delta_{\mathcal{S}, \mathbf{k}}$, we must first diagonalise (4.31) once again. For simplicity's sake we have chosen to write $\tan \alpha(\mathbf{k})$ as $t$ in the following expressions. Adopting the same formalism as in subsection 3.2.1 allows us to simply find the new eigenenergies:

$$
\begin{gathered}
E_{1}(\mathbf{k})=\frac{1}{\sqrt{2}} \sqrt{\left(\frac{1}{t}+t\right)^{2}\left|\Delta_{\mathcal{S}, \mathbf{k}}\right|^{2}+\xi_{1, \mathbf{k}}^{2}+\xi_{2, \mathbf{k}}^{2}+B_{\mathbf{k}}} \\
E_{2}(\mathbf{k})=\frac{1}{\sqrt{2}} \sqrt{\left(\frac{1}{t}+t\right)^{2}\left|\Delta_{\mathcal{S}, \mathbf{k}}\right|^{2}+\xi_{1, \mathbf{k}}^{2}+\xi_{2, \mathbf{k}}^{2}-B_{\mathbf{k}}} \\
E_{3}(\mathbf{k})=-E_{1}(\mathbf{k}) \\
E_{4}(\mathbf{k})=-E_{2}(\mathbf{k})
\end{gathered}
$$

where $B_{\mathbf{k}}$ is defined as

$$
\begin{aligned}
B_{\mathbf{k}}=\left(\left(\frac{1}{t}+t\right)^{4}\left|\Delta_{\mathcal{S}, \mathbf{k}}\right|^{4}-2\left|\Delta_{\mathcal{S}, \mathbf{k}}\right|^{2}\left(\xi_{1, \mathbf{k}}-\xi_{2, \mathbf{k}}\right)\left(\left(t^{2}-2-\frac{1}{t^{2}}\right) \xi_{1, \mathbf{k}}\right.\right. \\
\left.\left.+\left(t^{2}+2-\frac{1}{t^{2}}\right) \xi_{2, \mathbf{k}}\right)+\left(\xi_{1, \mathbf{k}}^{2}-\xi_{2, \mathbf{k}}^{2}\right)^{2}\right)^{\frac{1}{2}}
\end{aligned}
$$

We will also need the eigenspinors $\mathbf{w}_{n}(n=1,2,3,4)$ in order to express the expectation value $\left\langle c_{2,-\mathbf{k}} c_{1, \mathbf{k}}\right\rangle$ in (4.30). The eigenspinors however, as before in the previous chapter, are clumsy expressions that, for brevity, will not be written down here. We 
will also find that we only need two of these vectors for us to write our expectation value. In addition to this the form of our expectation value will also mirror that of (3.34) where we will have $\left\langle c_{2,-\mathbf{k}} c_{1, \mathbf{k}}\right\rangle=\Delta_{\mathcal{S}, \mathbf{k}} E V(\mathbf{k})$ for some complicated function $E V(\mathbf{k})$. We must find how to express the $c_{n, \mathbf{k}}$ operators in the new diagonalised basis as in (3.27) to find the form of $E V(\mathbf{k})$. So, as we did in (3.27), we may write for our $c_{n, \mathbf{k}}$ operators:

$$
\begin{aligned}
c_{1, \mathbf{k}} & =u_{1,1, \mathbf{k}} \gamma_{1, \mathbf{k}}+u_{1,2, \mathbf{k}} \gamma_{2, \mathbf{k}}+u_{1,3, \mathbf{k}} \gamma_{1,-\mathbf{k}}^{\dagger}+u_{1,4, \mathbf{k}} \gamma_{2,-\mathbf{k}}^{\dagger} \\
c_{2, \mathbf{k}} & =u_{2,1, \mathbf{k}} \gamma_{1, \mathbf{k}}+u_{2,2, \mathbf{k}} \gamma_{2, \mathbf{k}}+u_{2,3, \mathbf{k}} \gamma_{1,-\mathbf{k}}^{\dagger}+u_{2,4, \mathbf{k}} \gamma_{2,-\mathbf{k}}^{\dagger} \\
c_{1,-\mathbf{k}}^{\dagger} & =v_{1,1, \mathbf{k}} \gamma_{1, \mathbf{k}}+v_{1,2, \mathbf{k}} \gamma_{2, \mathbf{k}}+v_{1,3, \mathbf{k}} \gamma_{1,-\mathbf{k}}^{\dagger}+v_{1,4, \mathbf{k}} \gamma_{2,-\mathbf{k}}^{\dagger} \\
c_{2,-\mathbf{k}}^{\dagger} & =v_{2,1, \mathbf{k}} \gamma_{1, \mathbf{k}}+v_{2,2, \mathbf{k}} \gamma_{2, \mathbf{k}}+v_{2,3, \mathbf{k}} \gamma_{1,-\mathbf{k}}^{\dagger}+v_{2,4, \mathbf{k}} \gamma_{2,-\mathbf{k}}^{\dagger}
\end{aligned}
$$

Using these expressions and our knowledge of what terms in such an expansion survive we may neatly write:

$$
\begin{aligned}
\left\langle c_{2,-\mathbf{k}} c_{1, \mathbf{k}}\right\rangle & =\left\langle\Phi_{G S}\left|u_{1,3, \mathbf{k}} v_{2,3, \mathbf{k}}^{*} \gamma_{1,-\mathbf{k}} \gamma_{1,-\mathbf{k}}^{\dagger}+u_{1,4, \mathbf{k}} v_{2,4, \mathbf{k}}^{*} \gamma_{2,-\mathbf{k}} \gamma_{2,-\mathbf{k}}^{\dagger}\right| \Phi_{G S}\right\rangle \\
& =u_{1,3, \mathbf{k}} v_{2,3, \mathbf{k}}^{*}+u_{1,4, \mathbf{k}} v_{2,4, \mathbf{k}}^{*}
\end{aligned}
$$

The form of the expectation value, as mentioned before, is in the form $\left\langle c_{2,-\mathbf{k}} c_{1, \mathbf{k}}\right\rangle=$ $\Delta_{\mathcal{S}, \mathbf{k}} E V(\mathbf{k})$ where $E V(\mathbf{k})$ does not depend on the angle $\phi$. However, in contrast to the previous chapter, the expression for this expectation value (4.39) can be markedly simplified, but only after performing the $k$ integral in our resulting selfconsistency integral. What we find is that we can approximate a factor in the integral as a delta function, which is what simplifies the expression. So in order to optimise the use of space we will give the required forms of the needed eigenspinors after we find the result of our $k$ integral. In this instance we may now take our self-consistency integral to have the form

$$
\Delta_{\mathcal{S}}(\mathbf{k})=\sin 2 \alpha(\mathbf{k}) \operatorname{sign}(\mathbf{k} \cdot \mathbf{P}) V_{2} \int d k^{\prime 3} \mathbf{k} \cdot \mathbf{k}^{\prime} \sin 2 \alpha\left(\mathbf{k}^{\prime}\right) \operatorname{sign}\left(\mathbf{k}^{\prime} \cdot \mathbf{P}\right) \Delta_{\mathcal{S}}\left(\mathbf{k}^{\prime}\right) E V\left(\mathbf{k}^{\prime}\right)
$$

\subsubsection{Treating the Self-Consistency Equation}

Now that we have the self-consistency equation we may choose again, without loss of generality, to have $\mathbf{P}$ point along the $z$-direction. With this choice we may make 


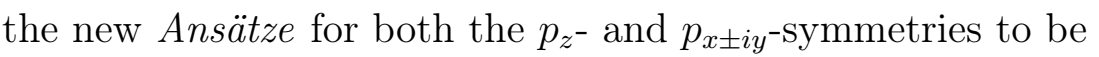

$$
\begin{aligned}
\Delta_{\mathcal{S}, z}(\mathbf{k}) & =\operatorname{sign}(\mathbf{k} \cdot \mathbf{P}) \sin 2 \alpha(\mathbf{k}) \frac{k_{z}}{k_{C}} \Delta_{\mathcal{S} 0}^{(0)} \\
& =\operatorname{sign}(\mathbf{k} \cdot \mathbf{P}) \sin 2 \alpha(\mathbf{k}) \frac{k}{k_{C}} \cos \theta \Delta_{\mathcal{S} 0}^{(0)}
\end{aligned}
$$

and

$$
\begin{aligned}
\Delta_{\mathcal{S}, x \pm i y}(\mathbf{k}) & =\operatorname{sign}(\mathbf{k} \cdot \mathbf{P}) \sin 2 \alpha(\mathbf{k}) \frac{k_{x} \pm i k_{y}}{k_{C}} e^{ \pm i \phi} \sin \theta \Delta_{\mathcal{S} 0}^{( \pm 1)} \\
& =\operatorname{sign}(\mathbf{k} \cdot \mathbf{P}) \sin 2 \alpha(\mathbf{k}) \frac{k}{k_{C}} \Delta_{\mathcal{S} 0}^{( \pm 1)}
\end{aligned}
$$

where $k_{C}$ is the magnitude of the $\mathbf{k}$ where the $d$ and $f$ bands intersect. This choice, as opposed to $k_{F}$, will be made clear once we consider the integral (4.40) in more depth. Starting with the $p_{z}$-symmetry case we can begin by changing the integral in (4.40) to spherical coordinates and by writing it in the following way:

$$
\begin{array}{r}
\operatorname{sign}(\mathbf{k} \cdot \mathbf{P}) \sin 2 \alpha(\mathbf{k}) \frac{k}{k_{C}} \cos \theta \Delta_{\mathcal{S} 0}^{(0)}=\sin 2 \alpha(\mathbf{k}) \operatorname{sign}(\mathbf{k} \cdot \mathbf{P}) \frac{k \Delta_{\mathcal{S} 0}^{(0)}}{k_{C}} V_{2} \frac{\Omega}{(2 \pi)^{3}} \\
\times \int d k^{\prime} \int_{0}^{\pi} d \theta^{\prime} \int_{0}^{2 \pi} d \phi^{\prime} k^{\prime 4} \sin \theta^{\prime} \cos \theta^{\prime} \sin ^{2} 2 \alpha\left(\mathbf{k}^{\prime}\right) \operatorname{sign}\left(\mathbf{k}^{\prime} \cdot \mathbf{P}\right)^{2} \\
\times\left(\cos \left(\phi-\phi^{\prime}\right) \sin \theta \sin \theta^{\prime}+\cos \theta \cos \theta^{\prime}\right) E V\left(k^{\prime}, \theta^{\prime}\right)
\end{array}
$$

We see that, just like in the previous integrals (3.42) and (3.43), the integral simplifies nicely as well as the integral in $\phi$ being straightforward. This allows us to write

$$
1=V_{2} \frac{\Omega}{(2 \pi)^{3}} \int d k^{\prime} \int_{0}^{\pi} d \theta^{\prime} k^{\prime 4} \sin \theta^{\prime} \cos ^{2} \theta^{\prime} \sin ^{2} 2 \alpha\left(\mathbf{k}^{\prime}\right) E V\left(k^{\prime}, \theta^{\prime}\right)
$$

cancelling out the leftover $\cos \theta$ factor on both sides after the result of the $\phi$ integral. The only difference now between this integral and the one in (3.44) is the $\sin ^{2} 2 \alpha(\mathbf{k})$ factor. If we consider the form of such a factor as $\sin 2 \alpha(\mathbf{k})=2 \sin \alpha(\mathbf{k}) \cos \alpha(\mathbf{k})$ then from our knowledge of the $\sin \alpha(\mathbf{k})$ and $\cos \alpha(\mathbf{k})$ functions, as in figure 4.1 we must conclude that it will be some sort of peaked function about the $k_{C}$, the crossing point. We have treated the $\sin ^{2} 2 \alpha(\mathbf{k})$ term separately in appendix B which leads us to find that it may be approximated as a delta function. For convenience, as explained in B, we introduce the wavevector scaling

$$
\mathbf{k}=\tilde{\mathbf{k}} k_{C}
$$


where $k_{C}$ is the wavevector where the un-hybridised bands intersect, i.e. $k_{C}=$

$$
k_{C}=\sqrt{\frac{2 m_{d \uparrow}\left(\varepsilon_{f b}-\varepsilon_{d, 0}\right)}{\hbar^{2}}} .
$$

With this new wavevector scaling we now find it reasonable to approximate $\sin ^{2} 2 \alpha(\tilde{\mathbf{k}})=$ $\sin ^{2} 2 \alpha(\tilde{k})$ as

$$
\sin ^{2} 2 \alpha(\tilde{k}) \approx \frac{1}{\pi} \zeta|\cos \theta| \delta(\tilde{k}-1)
$$

where $\zeta=\frac{P k_{C}}{\varepsilon_{f b}-\varepsilon_{d, 0}}=\frac{P k_{C}}{\left(\Delta E_{0}\right)}$. This is due to the function $\sin ^{2} 2 \alpha(\tilde{k})$ resembling a Lorentzian curve for $\zeta^{2} \ll 1$.

With this result we turn back to our original problem in (4.44). Now considering only the integral in $k$ we will have

$$
\int d k k^{4} \sin ^{2} 2 \alpha(k) E V(k, \theta) \rightarrow \int d \tilde{k} k_{C}^{5} \tilde{k}^{4} \sin ^{2} 2 \alpha\left(\tilde{k} k_{C}\right) E V\left(\tilde{k} k_{C}, \theta\right)
$$

by making the substitution $k=\tilde{k} k_{C}$. Now we recognise that $\sin ^{2} 2 \alpha\left(\tilde{k} k_{C}\right)$ is simply our function with the correct wavevector scaling such that we may approximate it with a delta function. By using (4.47) we may now rewrite (4.48) to

$$
\frac{1}{\pi} k_{C}^{5} \zeta \int d \tilde{k} \tilde{k}^{4}|\cos \theta| \delta(\tilde{k}-1) E V\left(\tilde{k} k_{C}, \theta\right)
$$

which can be simply evaluated to

$$
\frac{1}{\pi} k_{C}^{5} \zeta|\cos \theta| E V\left(k_{C}, \theta\right)=\frac{2 k_{C} P}{\pi\left(\Delta E_{0}\right)} k_{C}^{5}|\cos \theta| E V\left(k_{C}, \theta\right)
$$

Putting this result back into the self-consistency integral now we may finally write

$$
1=\frac{2 V_{2} k_{C}^{6} P \Omega}{\pi(2 \pi)^{2}\left(\Delta E_{0}\right)} \int_{0}^{\pi} d \theta^{\prime} \sin \theta^{\prime} \cos ^{2} \theta^{\prime}\left|\cos \theta^{\prime}\right| E V\left(k_{C}, \theta^{\prime}\right)
$$

This can be simplified by making the substitution $u=\cos \theta^{\prime}$ which gives

$$
1=\frac{2 V_{2} k_{C}^{6} P \Omega}{\pi(2 \pi)^{2}\left(\Delta E_{0}\right)} \int_{-1}^{1} d u|u| u^{2} E V\left(k_{C}, u\right)
$$

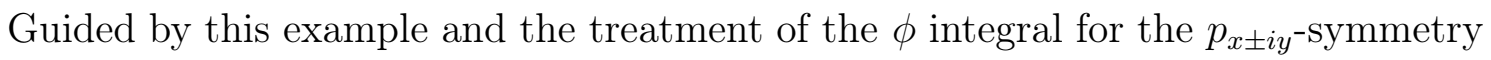
case seen before in chapter 3 we may also write for the $p_{x \pm i y}$-symmetry pair potential 
this self-consistency equation:

$$
1=\frac{V_{2} k_{C}^{6} P \Omega}{\pi(2 \pi)^{2}\left(\Delta E_{0}\right)} \int_{-1}^{1} d u|u|\left(1-u^{2}\right) E V\left(k_{C}, u\right) .
$$

With this result care must be taken however since we only expect non-zero contributions to the pairing in the thin shell around the Fermi level. Thus this treatment is only valid when the avoided crossing of the two bands is within the range of the thin shell around the Fermi energy.

\subsection{Results}

As we have seen here the approximation of the $\sin ^{2} 2 \alpha(k)$ factor as a delta function has vastly simplified the integral and simplified $E V$ since now it must be taken at $k_{C}$. This allows us to take any $\tan \alpha(\mathbf{k})$ terms to be 1 which simplifies the expressions in how they are expressed and internally as well, i.e. the functions $E_{1, \mathbf{k}}$ will become simpler as well. Writing these eigenspinors so that we might express $E V$ in full we will have:

$$
\mathbf{w}_{3}=\frac{1}{\mathcal{N}_{3}}\left[\begin{array}{c}
\frac{\left(\xi_{2, k_{C}}^{2}+4\left|\Delta_{\mathcal{S}, k_{C}}\right|^{2}-E_{1, k_{C}}^{2}\right)\left(\xi_{1, k_{C}}+\xi_{2, k_{C}}-2 E_{1, k_{C}}\right)}{\left(\xi_{1, k_{C}}-\xi_{2, k_{C}}\right)\left(3 \xi_{1, k_{C}}+\xi_{2, k_{C}}\right)} \\
e^{-2 i \theta} \operatorname{sign}\left(k_{C} P u\right)\left|\Delta_{\mathcal{S}, k_{C}}\right|^{2} \frac{{ }_{\xi_{1, k_{C}}+\xi_{2, k_{C}}-2 E_{1, k_{C}}}{ }_{\left(E_{1, k_{C}}-\xi_{1, k_{C}}\right)\left(E_{1, k_{C}}+\xi_{2, k_{C}}\right)}}{e^{-i \theta} \operatorname{sign}\left(k_{C} P u\right) \Delta_{\mathcal{S}, k_{C}}^{*} \frac{\xi_{2, k_{C}}-E_{1, k_{C}}}{\xi_{1, k_{C}}-E_{1, k_{C}}}} \\
\Delta_{\mathcal{S}, k_{C}}^{*}
\end{array}\right]
$$

and

$$
\mathbf{w}_{4}=\frac{1}{\mathcal{N}_{4}}\left[\begin{array}{c}
\frac{\left(\xi_{2, k_{C}}^{2}+4\left|\Delta_{\mathcal{S}, k_{C}}\right|^{2}-E_{2, k_{C}}^{2}\right)\left(\xi_{1, k_{C}}+\xi_{2, k_{C}}-2 E_{2, k_{C}}\right)}{\left(\xi_{1, k_{C}}-\xi_{2, k_{C}}\right)\left(3 \xi_{1, k_{C}}+\xi_{2, k_{C}}\right)} \\
e^{-2 i \theta} \operatorname{sign}\left(k_{C} P u\right)\left|\Delta_{\mathcal{S}, k_{C}}\right|^{2} \frac{\xi_{1, k_{C}}+\xi_{2, k_{C}}-2 E_{2, k_{C}}}{\left(E_{2, k_{C}}-\xi_{1, k_{C}}\right)\left(E_{2, k_{C}}+\xi_{2, k_{C}}\right)} \\
e^{-i \theta} \operatorname{sign}\left(k_{C} P u\right) \Delta_{\mathcal{S}, k_{C}}^{*} \frac{\xi_{2, k_{C}}-E_{2, k_{C}}}{\xi_{1, k_{C}}-E_{2, k_{C}}} \\
\Delta_{\mathcal{S}, k_{C}}^{*}
\end{array}\right]
$$

where $\mathcal{N}_{3}$ and $\mathcal{N}_{4}$ are the norms of the vector. These norms are founf to be:

$$
\begin{aligned}
\mathcal{N}_{3}^{2}= & \left(\frac{\left(\xi_{2, k_{C}}^{2}+4\left|\Delta_{\mathcal{S}, k_{C}}\right|^{2}-E_{1, k_{C}}^{2}\right)\left(\xi_{1, k_{C}}+\xi_{2, k_{C}}-2 E_{1, k_{C}}\right)}{\left(\xi_{1, k_{C}}-\xi_{2, k_{C}}\right)\left(3 \xi_{1, k_{C}}+\xi_{2, k_{C}}\right)}\right)^{2} \\
& \left.+\left|\Delta_{\mathcal{S}, k_{C}}\right|^{4} \mid \frac{\xi_{1, k_{C}}+\xi_{2, k_{C}}-2 E_{1, k_{C}}}{\left(E_{1, k_{C}}-\xi_{1, k_{C}}\right)\left(E_{1, k_{C}}+\xi_{2, k_{C}}\right)}\right)^{2}+\left|\Delta_{\mathcal{S}, k_{C}}\right|^{2}\left(\frac{\xi_{2, k_{C}}-E_{1, k_{C}}}{\xi_{1, k_{C}}-E_{1, k_{C}}}\right)^{2}+\left|\Delta_{\mathcal{S}, k_{C}}\right|^{2}
\end{aligned}
$$


and

$$
\begin{aligned}
\mathcal{N}_{4}^{2}= & \left(\frac{\left(\xi_{2, k_{C}}^{2}+4\left|\Delta_{\mathcal{S}, k_{C}}\right|^{2}-E_{2, k_{C}}^{2}\right)\left(\xi_{1, k_{C}}+\xi_{2, k_{C}}-2 E_{2, k_{C}}\right)}{\left(\xi_{1, k_{C}}-\xi_{2, k_{C}}\right)\left(3 \xi_{1, k_{C}}+\xi_{2, k_{C}}\right)}\right)^{2} \\
& \left.+\left|\Delta_{\mathcal{S}, k_{C}}\right|^{4} \mid \frac{\xi_{1, k_{C}}+\xi_{2, k_{C}}-2 E_{2, k_{C}}}{\left(E_{2, k_{C}}-\xi_{1, k_{C}}\right)\left(E_{2, k_{C}}+\xi_{2, k_{C}}\right)}\right)^{2}+\left|\Delta_{\mathcal{S}, k_{C}}\right|^{2}\left(\frac{\xi_{2, k_{C}}-E_{2, k_{C}}}{\xi_{1, k_{C}}-E_{2, k_{C}}}\right)^{2}+\left|\Delta_{\mathcal{S}, k_{C}}\right|^{2} .
\end{aligned}
$$

Since everything is taken at $k_{C}$ now the expressions for $\xi_{n}$ reduce to $\xi_{1, k_{C}}=\frac{1}{2}\left(\varepsilon_{d, k_{C}}+\right.$ $\left.\varepsilon_{f b}+2 k_{C} P|u|\right)$ and $\xi_{2, k_{C}}=\frac{1}{2}\left(\varepsilon_{d, k_{C}}+\varepsilon_{f b}-2 k_{C} P|u|\right)$. As well as this the eigenerergies can be shown to also reduce to

$$
\begin{aligned}
E_{1, k_{C}}=\frac{1}{\sqrt{2}}\left(4\left|\Delta_{\mathcal{S}, k_{C}}\right|^{2}\right. & +\xi_{1, k_{C}}^{2}+\xi_{2, k_{C}}^{2} \\
& \left.+2 \sqrt{4\left|\Delta_{\mathcal{S}, k_{C}}\right|^{4}+k_{C}^{2} P^{2} u^{2}\left(4\left|\Delta_{\mathcal{S}, k_{C}}\right|^{2}+\left(\varepsilon_{d, k_{C}}+\varepsilon_{f b}\right)^{2}\right)}\right)^{\frac{1}{2}}
\end{aligned}
$$

and

$$
\begin{aligned}
E_{2, k_{C}}=\frac{1}{\sqrt{2}}\left(4\left|\Delta_{\mathcal{S}, k_{C}}\right|^{2}+\xi_{1, k_{C}}^{2}+\xi_{2, k_{C}}^{2}\right. & \\
& \left.\quad-2 \sqrt{4\left|\Delta_{\mathcal{S}, k_{C}}\right|^{4}+k_{C}^{2} P^{2} u^{2}\left(4\left|\Delta_{\mathcal{S}, k_{C}}\right|^{2}+\left(\varepsilon_{d, k_{C}}+\varepsilon_{f b}\right)^{2}\right)}\right)^{\frac{1}{2}}
\end{aligned}
$$

Now these eigenspinors can give us the expression for $E V\left(k_{C}, u\right)$ which is

$$
\begin{aligned}
& \left\langle c_{2,-\mathbf{k}} c_{1, \mathbf{k}^{\prime}}\right\rangle=\Delta_{\mathcal{S}, k_{C}}\left[\frac{\left(\xi_{2, k_{C}}^{2}+4\left|\Delta_{\mathcal{S}, k_{C}}\right|^{2}-E_{1, k_{C}}^{2}\right)\left(\xi_{1, k_{C}}+\xi_{2, k_{C}}-2 E_{1, k_{C}}\right)}{\mathcal{N}_{3}^{2}\left(\xi_{1, k_{C}}-\xi_{2, k_{C}}\right)\left(3 \xi_{1, k_{C}}+\xi_{2, k_{C}}\right)}+\right. \\
& \left.\frac{\left(\xi_{2, k_{C}}^{2}+4\left|\Delta_{\mathcal{S}, k_{C}}\right|^{2}-E_{2, k_{C}}^{2}\right)\left(\xi_{1, k_{C}}+\xi_{2, k_{C}}-2 E_{2, k_{C}}\right)}{\mathcal{N}_{4}^{2}\left(\xi_{1, k_{C}}-\xi_{2, k_{C}}\right)\left(3 \xi_{1, k_{C}}+\xi_{2, k_{C}}\right)}\right] \\
& =\Delta_{\mathcal{S}, k_{C}} E V\left(k_{C}, u\right)
\end{aligned}
$$

While it is not possible to analytically solve the integral with $E V$ in this form, preliminary numerical results indicate that the integral gives some positive number for appropriate values of $\Delta_{0}$. They also seem to suggest that the integral attains some maximum value at some $\Delta_{0}$. This means that, in principle, it is possible to solve this self-consistency equation if the effective coupling strength is great enough. Thus indicating that this pairing does indeed exist and may play a key role in the superconducting state of $\mathrm{SmN}$. Since this pairing can exist now we can look at the 
nature of our order parameters from our Ansätze (4.41) and (4.42). We see that both order parameters are even in $\mathbf{k}$, precluding it from triplet pairing, which is interesting since we started from only triplet pairing. The order parameters also have a $\sin 2 \alpha(\mathbf{k})$ prefactor which is peaked at wavevectors close to the avoided crossing. This means that the pairing amplitudes are peaked functions around the intersection point of the $d$ and $f$ bands, or in other words, the pairing amplitudes is significant only in the region of $\mathbf{k}$-space around the avoided crossing of the mixed bands. The fact that this singlet-like pairing can exist due to the triplet pairing in the $d$ band could be the reason the superconductivity in $\mathrm{SmN}$ survives disorder; there is some contribution to the triplet pairing which is singlet in the mixed band indices. This is a very exciting result which may offer up an answer to the existence of superconductivity in $\mathrm{SmN}$ even in the presence of disorder.

\subsection{Conclusions}

In this chapter we have considered how the triplet pairing in the $5 d$ band acts in the basis of the mixed bands. This shift of basis essentially 'mixes' the interaction term into different intra- and inter-band pairing in the mixed bands. By applying the mean-field approximation to this new scenario we saw the emergence of some total pairing $\Delta_{\mathcal{S}}$ that turned out to be even in $\mathbf{k}$ with a form similar to that of other singlet pairings. With this form of the pairing we were able to create the self-consistency equation and made significant in-roads into the solution of such an equation. The findings from our investigation into the self-consistency equation allowed us to state the existence of such an even parity singlet pairing term between the mixed bands $\xi_{1, \mathbf{k}}$ and $\xi_{2, \mathbf{k}}$. This pairing exists near the avoided crossing of the two mixed bands and serves to pair electrons from each band together across this avoided crossing. The emergence of such a pairing from the postulated triplet pairing in the $d$ band could be a reason for the observation of superconductivity in $\mathrm{SmN}$, even in the presence of disorder. To properly make this claim, more in depth study is required and disorder must be added to the system, however, this finding is an exciting step forward in our understanding of this material's system. 


\section{Chapter 5}

\section{Summary, Conclusions, and Outlook}

The aim of this thesis was to understand in more depth the superconductivity of SmN. Towards this end we have explored a low energy approximation of SmN's bandstructure which consisted of the Sm $4 f$ band and N $5 d$ bands. These bands lay close to the Fermi level and interacted strongly with one another to create hybridisation between the bands. With this picture in mind for our system we postulated the existence for superconducting pairing between electrons due to the electron-phonon interaction, where a travelling phonon in the crystal displaces the crystal in such a way to effectively attract two electrons. We performed a calculation for long wavelength phonons that detailed how they would act in the crystal, as well as the resultant form such an interaction would have.

With this knowledge of the material in place we formulated our model with the electron-phonon interaction to form the effective Hamiltonian of our system. This lead us to the assertion that, in the absence of disorder and/or impurities, the most likely place for superconducting pairing would be in the $d$ band. Taking inspiration from BCS superconducting theory and spinless $p$-wave theory we performed the mean-field approximation on our system and diagonalised the resultant system to form our self-consistency equation for the superconducting pairing $\Delta_{d}$. After many considerations on how to reduce the resulting self-consistency integrals we found solutions for the self-consistency equation in the limits for large and small $\Delta_{d}$ in comparison to the other energy scales at play. We investigated two different cases, one more successfully than the other. The first, and more successful, was the case where the material has been doped such that the Fermi level is the same as the $f$ band. Analytic solutions in this case showed that for large $\Delta_{d}$ the gap equation was linear in the effective coupling strength $\lambda$. In the small limit however, solutions for the gap equation for all three $p$-wave symmetries showed that there would only be a solution for sufficiently large effective coupling strengths. Physically this means 
that if the effective coupling strength is insufficient then the superconducting state will not be established. The second case we explored was the more general case where the Fermi level did not coincide with the $f$ band. In this case for large $\Delta_{d}$ we found the same solutions as in the first case but we could not find analytic solutions for the small $\Delta_{d}$ limit. However, our numerical investigations showed that in this limit the self-consistency integral behaves as $-\ln \left(\Delta_{0}\right)$. This would lead to solutions of the gap equation that are exponential in the effective coupling strength, which is the same form as the pairing found for the single band case, up to constant factors.

On the other hand we attempted the same treatment of superconducting pairing of the material in the mixed bands, rather than in the un-hybridised $d$ and $f$ bands. The motivation for redoing the analysis was to search for new pairing that would turn out to be more robust in the presence of disorder than just pure triplet pairing. Keeping the pairing still within the $d$ band, the effective interaction was mixed into hybrid intra- and inter-band pairing when shifted to the mixed band basis. Once the mean-field approximation had been applied to this new scenario we uncovered the existence of a pairing $\Delta_{\mathcal{S}}$ that was both even in the wavevector $\mathbf{k}$ and had a form reminiscent of inter-band pairing between the two mixed bands. The definition of this pairing had the added property of only existing near the avoided crossing of the mixed bands, the place where the $d$ and $f$ bands would have crossed in the absence of hybridisation. From this point we once again expressed the self-consistency equation as a set of similar integrals to before. Sadly no analytic work could be done for solutions of the resultant integrals, however, preliminary numerical results showed that in principle the self-consistency equation could be solved, provided once again that the effective coupling strength was large enough. This indicated that such pairing is possible to exist within the material and may even be resistant to disorder due to its singlet-like form.

The conclusions that we can garner from our results is that in the absence of disorder and/or impurities we do in fact find triplet superconducting pairing which we believe to be $p$-wave since the resultant symmetries all have defined solutions. We also know that as a result of this triplet pairing we can find, given the Fermilevel is close enough to the avoided crossing, a contribution to this pairing which is singlet in the mixed band indices. This indicates pairing between electrons in different bands across the avoided crossing. The existence of such pairing may be the key to superconductivity surviving in the presence of disorder and is an exciting new step into the origins of SmN's superconductivity.

Moving forward into the future then we envision several avenues of further research. The first being the completion of analytic solutions in the small $\Delta_{d}$ limit 
for the general case in the $d$ and $f$ band basis where the $f$ band is different to the Fermi level. The next is looking in greater depth at the self-consistency equation for the case of the singlet-like pairing between the mixed bands since this could hold the key to the robustness of the superconductivity in the presence of impurities. The final immediate avenue of exploration is the introduction of impurities and/or disorder into the system to see if in fact any pairing in the $d$ band survives. The form of such an addition to the Hamiltonian could easily be of the form given in by Asano et al. [79] which describes random non-magnetic impurities in the basis of the un-hybridised bands $\{|d \uparrow\rangle,|f\rangle\}$ :

$$
H_{i m p}=V_{i m p}(\mathbf{r})\left[\begin{array}{cccc}
1 & e^{i \theta} & 0 & 0 \\
e^{-i \theta} & 1 & 0 & 0 \\
0 & 0 & -1 & -e^{-i \theta} \\
0 & 0 & -e^{i \theta} & -1
\end{array}\right] .
$$

This equation captures both intra- and inter-band scattering and can be seen when written as the product of the Pauli spin matrices in the electron-hole $\left(\tau_{i}\right)$ and two band spaces $\left(\rho_{i}\right)$ :

$$
H_{i m p}=V_{i m p}(\mathbf{r}) \hat{\tau}_{3} \hat{\rho}_{0}+V_{i m p}(\mathbf{r}) \hat{A}
$$

where $\hat{A}=\cos \theta \hat{\tau}_{3} \hat{\rho}_{1}-\sin \theta \hat{\tau}_{3} \hat{\rho}_{2}$, the first term represents intra-band pairing, and the second term represents inter-band pairing. The inclusion of this contribution in the system would shed light onto the existence of the pairing we have found previously in a more realistic system.

Further long term avenues of investigation include looking at the interplay between this material and Gadolinium Nitride (GdN) in a heterostructure, which has been reported [35] to enhance the superconducting state of SmN. Understanding this mechanism may lead to applications in pursuing higher critical temperatures in similar materials. 
Appendices 



\section{Appendix A}

\section{Simplification of the Expectation Value}

Starting with the expression (3.60) we define a new variable to help us with the simplification. Let us call it $r_{\mathbf{k}}$ where

$$
r_{\mathbf{k}}^{2}=4(\mathbf{k} \cdot \mathbf{P})^{2}+\hbar^{2} v_{F}^{2} q^{2}+\left|\Delta_{d}\right|^{2}
$$

Now we can rewrite (3.60) and (3.61) as:

$$
\mathcal{N}_{1}=\left(\hbar^{2} v_{F}^{2} q^{2}+\left|\Delta_{d}\right|^{2}\right)\left(r_{\mathbf{k}}^{2}+r_{\mathbf{k}} \sqrt{\hbar^{2} v_{F}^{2} q^{2}+\left|\Delta_{d}\right|^{2}}\right)-2 r_{\mathbf{k}} E_{1, \mathbf{k}} \hbar v_{F} q \sqrt{\hbar^{2} v_{F}^{2} q^{2}+\left|\Delta_{d}\right|^{2}}
$$

and

$$
\mathcal{N}_{2}=\left(\hbar^{2} v_{F}^{2} q^{2}+\left|\Delta_{d}\right|^{2}\right)\left(r_{\mathbf{k}}^{2}-r_{\mathbf{k}} \sqrt{\hbar^{2} v_{F}^{2} q^{2}+\left|\Delta_{d}\right|^{2}}\right)+2 r_{\mathbf{k}} E_{2, \mathbf{k}} \hbar v_{F} q \sqrt{\hbar^{2} v_{F}^{2} q^{2}+\left|\Delta_{d}\right|^{2}}
$$

We can also rewrite both $E_{1, \mathbf{k}}$ and $E_{2, \mathbf{k}}$ as

$$
\begin{aligned}
E_{1}(\mathbf{k}) & \left.=\frac{1}{\sqrt{2}} \sqrt{\frac{1}{2}\left(r_{\mathbf{k}}^{2}+\hbar^{2} v_{F}^{2} q^{2}+\left|\Delta_{d}\right|^{2}\right.}\right)+r_{\mathbf{k}} \sqrt{\hbar^{2} v_{F}^{2} q^{2}+\left|\Delta_{d}\right|^{2}} \\
& =\frac{1}{2} \sqrt{\left(r_{\mathbf{k}}+\sqrt{\hbar^{2} v_{F}^{2} q^{2}+\left|\Delta_{d}\right|^{2}}\right)^{2}} \\
& =\frac{1}{2}\left(r_{\mathbf{k}}+\sqrt{\hbar^{2} v_{F}^{2} q^{2}+\left|\Delta_{d}\right|^{2}}\right)
\end{aligned}
$$


and

$$
\begin{aligned}
E_{2}(\mathbf{k}) & =\frac{1}{\sqrt{2}} \sqrt{\frac{1}{2}\left(r_{\mathbf{k}}^{2}+\hbar^{2} v_{F}^{2} q^{2}+\left|\Delta_{d}\right|^{2}\right)}-r_{\mathbf{k}} \sqrt{\hbar^{2} v_{F}^{2} q^{2}+\left|\Delta_{d}\right|^{2}} \\
& =\frac{1}{2} \sqrt{\left(r_{\mathbf{k}}-\sqrt{\hbar^{2} v_{F}^{2} q^{2}+\left|\Delta_{d}\right|^{2}}\right)^{2}} \\
& =\frac{1}{2}\left(r_{\mathbf{k}}-\sqrt{\hbar^{2} v_{F}^{2} q^{2}+\left|\Delta_{d}\right|^{2}}\right)
\end{aligned}
$$

Working only on (A.2) for now, we see that

$$
\begin{gathered}
\mathcal{N}_{1}=r_{\mathbf{k}}\left(\hbar^{2} v_{F}^{2} q^{2}+\left|\Delta_{d}\right|^{2}\right)\left(r_{\mathbf{k}}+\sqrt{\hbar^{2} v_{F}^{2} q^{2}+\left|\Delta_{d}\right|^{2}}\right) \\
-\left(r_{\mathbf{k}}+\sqrt{\hbar^{2} v_{F}^{2} q^{2}+\left|\Delta_{d}\right|^{2}}\right) \hbar v_{F} q r_{\mathbf{k}} \sqrt{\hbar^{2} v_{F}^{2} q^{2}+\left|\Delta_{d}\right|^{2}} \\
=r_{\mathbf{k}} \sqrt{\hbar^{2} v_{F}^{2} q^{2}+\left|\Delta_{d}\right|^{2}}\left(r_{\mathbf{k}}+\sqrt{\hbar^{2} v_{F}^{2} q^{2}+\left|\Delta_{d}\right|^{2}}\right)\left(\sqrt{\hbar^{2} v_{F}^{2} q^{2}+\left|\Delta_{d}\right|^{2}}-\hbar v_{F} q\right)
\end{gathered}
$$

Working on the numerator above $\mathcal{N}_{1}$ in (3.59) now we find:

$$
\begin{gathered}
E_{1, \mathbf{k}}\left(E_{1, \mathbf{k}}^{2}-(\mathbf{k} \cdot \mathbf{P})^{2}-E_{1, \mathbf{k}} \hbar v_{F} q\right)= \\
=\frac{1}{2}\left(r_{\mathbf{k}}+\sqrt{\hbar^{2} v_{F}^{2} q^{2}+\left|\Delta_{d}\right|^{2}}\right)\left(\frac{1}{2}\left(\hbar^{2} v_{F}^{2} q^{2}+\left|\Delta_{d}\right|^{2}+\sqrt{\hbar^{2} v_{F}^{2} q^{2}+\left|\Delta_{d}\right|^{2}}\right)\right. \\
\left.-\frac{1}{2} \hbar v_{F} q\left(r_{\mathbf{k}}+\sqrt{\hbar^{2} v_{F}^{2} q^{2}+\left|\Delta_{d}\right|^{2}}\right)\right) \\
=\frac{1}{4}\left(r_{\mathbf{k}}+\sqrt{\hbar^{2} v_{F}^{2} q^{2}+\left|\Delta_{d}\right|^{2}}\right)\left(\sqrt{\hbar^{2} v_{F}^{2} q^{2}+\left|\Delta_{d}\right|^{2}}\left(\sqrt{\hbar^{2} v_{F}^{2} q^{2}+\left|\Delta_{d}\right|^{2}}+r_{\mathbf{k}}\right)\right. \\
\left.-\hbar v_{F} q\left(\sqrt{\hbar^{2} v_{F}^{2} q^{2}+\left|\Delta_{d}\right|^{2}}+r_{\mathbf{k}}\right)\right) \\
=\frac{1}{4}\left(r_{\mathbf{k}}+\sqrt{\hbar^{2} v_{F}^{2} q^{2}+\left|\Delta_{d}\right|^{2}}\right)^{2}\left(\sqrt{\hbar^{2} v_{F}^{2} q^{2}+\left|\Delta_{d}\right|^{2}}-\hbar v_{F} q\right)
\end{gathered}
$$

Putting this together then we find:

$$
\begin{gathered}
\frac{E_{1, \mathbf{k}}\left(E_{1, \mathbf{k}}^{2}-(\mathbf{k} \cdot \mathbf{P})^{2}-E_{1, \mathbf{k}} \hbar v_{F} q\right)}{\mathcal{N}_{1}^{2}} \\
=\frac{r_{\mathbf{k}}+\sqrt{\hbar^{2} v_{F}^{2} q^{2}+\left|\Delta_{d}\right|^{2}}}{4 r_{\mathbf{k}} \sqrt{\hbar^{2} v_{F}^{2} q^{2}+\left|\Delta_{d}\right|^{2}}}
\end{gathered}
$$


Performing this same procedure for the other terms we find:

$$
\begin{gathered}
\mathcal{N}_{2}=r_{\mathbf{k}}\left(\hbar^{2} v_{F}^{2} q^{2}+\left|\Delta_{d}\right|^{2}\right)\left(r_{\mathbf{k}}-\sqrt{\hbar^{2} v_{F}^{2} q^{2}+\left|\Delta_{d}\right|^{2}}\right) \\
+\left(r_{\mathbf{k}}-\sqrt{\hbar^{2} v_{F}^{2} q^{2}+\left|\Delta_{d}\right|^{2}}\right) \hbar v_{F} q r_{\mathbf{k}} \sqrt{\hbar^{2} v_{F}^{2} q^{2}+\left|\Delta_{d}\right|^{2}} \\
=r_{\mathbf{k}} \sqrt{\hbar^{2} v_{F}^{2} q^{2}+\left|\Delta_{d}\right|^{2}}\left(r_{\mathbf{k}}-\sqrt{\hbar^{2} v_{F}^{2} q^{2}+\left|\Delta_{d}\right|^{2}}\right)\left(\sqrt{\hbar^{2} v_{F}^{2} q^{2}+\left|\Delta_{d}\right|^{2}}+\hbar v_{F} q\right)
\end{gathered}
$$

with the numerator above $\mathcal{N}_{2}$ in (3.59) giving:

$$
\begin{gathered}
E_{2, \mathbf{k}}\left(E_{2, \mathbf{k}}^{2}-(\mathbf{k} \cdot \mathbf{P})^{2}-E_{2, \mathbf{k}} \hbar v_{F} q\right)= \\
=\frac{1}{2}\left(r_{\mathbf{k}}-\sqrt{\hbar^{2} v_{F}^{2} q^{2}+\left|\Delta_{d}\right|^{2}}\right)\left(\frac{1}{2}\left(\hbar^{2} v_{F}^{2} q^{2}+\left|\Delta_{d}\right|^{2}-\sqrt{\hbar^{2} v_{F}^{2} q^{2}+\left|\Delta_{d}\right|^{2}}\right)\right. \\
\left.-\frac{1}{2} \hbar v_{F} q\left(r_{\mathbf{k}}-\sqrt{\hbar^{2} v_{F}^{2} q^{2}+\left|\Delta_{d}\right|^{2}}\right)\right) \\
=\frac{1}{4}\left(r_{\mathbf{k}}-\sqrt{\hbar^{2} v_{F}^{2} q^{2}+\left|\Delta_{d}\right|^{2}}\right)\left(\sqrt{\hbar^{2} v_{F}^{2} q^{2}+\left|\Delta_{d}\right|^{2}}\left(\sqrt{\hbar^{2} v_{F}^{2} q^{2}+\left|\Delta_{d}\right|^{2}}-r_{\mathbf{k}}\right)\right. \\
\left.+\hbar v_{F} q\left(\sqrt{\hbar^{2} v_{F}^{2} q^{2}+\left|\Delta_{d}\right|^{2}}-r_{\mathbf{k}}\right)\right) \\
=-\frac{1}{4}\left(r_{\mathbf{k}}-\sqrt{\hbar^{2} v_{F}^{2} q^{2}+\left|\Delta_{d}\right|^{2}}\right)^{2}\left(\sqrt{\hbar^{2} v_{F}^{2} q^{2}+\left|\Delta_{d}\right|^{2}}+\hbar v_{F} q\right)
\end{gathered}
$$

Putting this together then we find:

$$
\begin{gathered}
\frac{E_{2, \mathbf{k}}\left(E_{2, \mathbf{k}}^{2}-(\mathbf{k} \cdot \mathbf{P})^{2}-E_{2, \mathbf{k}} \hbar v_{F} q\right)}{\mathcal{N}_{2}^{2}} \\
=-\frac{r_{\mathbf{k}}-\sqrt{\hbar^{2} v_{F}^{2} q^{2}+\left|\Delta_{d}\right|^{2}}}{4 r_{\mathbf{k}} \sqrt{\hbar^{2} v_{F}^{2} q^{2}+\left|\Delta_{d}\right|^{2}}}
\end{gathered}
$$

Finally we can put (A.8) and (A.11) together to find the result given in (3.62):

$$
\begin{aligned}
E V(\mathbf{k})= & \frac{r_{\mathbf{k}}+\sqrt{\hbar^{2} v_{F}^{2} q^{2}+\left|\Delta_{d}\right|^{2}}}{4 r_{\mathbf{k}} \sqrt{\hbar^{2} v_{F}^{2} q^{2}+\left|\Delta_{d}\right|^{2}}}-\frac{r_{\mathbf{k}}-\sqrt{\hbar^{2} v_{F}^{2} q^{2}+\left|\Delta_{d}\right|^{2}}}{4 r_{\mathbf{k}} \sqrt{\hbar^{2} v_{F}^{2} q^{2}+\left|\Delta_{d}\right|^{2}}} \\
& =\frac{1}{2 r_{\mathbf{k}}}=\frac{1}{2 \sqrt{4(\mathbf{k} \cdot \mathbf{P})^{2}+\hbar^{2} v_{F}^{2} q^{2}+\left|\Delta_{d}\right|^{2}}}
\end{aligned}
$$




\section{Appendix B}

\section{Treating $\sin 2 \alpha(\mathbf{k})$}

To properly characterise this function it will be convenient to choose some set of energy and wavevector scaling to simplify the function. Since $\sin 2 \alpha(\mathbf{k})=2 \sin \alpha(\mathbf{k}) \cos \alpha(\mathbf{k})$ then it will be easier to first work on $\sin \alpha(\mathbf{k})$. We start by writing:

$$
\sin \alpha(\mathbf{k})=\frac{1}{\sqrt{2}} \sqrt{1-\frac{\Delta E}{E_{\mathbf{k}}}}
$$

with

$$
\Delta E=\varepsilon_{d, \mathbf{k}}-\varepsilon_{f b}=\varepsilon_{d, 0}+\frac{\hbar^{2}}{2 m_{d \uparrow}} k^{2}-\varepsilon_{f b}
$$

We will scale both $\Delta E$ and $E_{\mathbf{k}}$ separately, however, we will find that the scaling constants used will turn out to be the same. Beginning with $\Delta E$ we presume that $\Delta E$ and $\mathbf{k}$ can be written as

$$
\mathbf{k}=\tilde{\mathbf{k}} k_{r}, \quad \Delta E=\tilde{\Delta E} \Delta E_{r}
$$

where $\tilde{\mathbf{k}}$ and $\tilde{\Delta E}$ are dimensionless. Then (B.3) becomes:

$$
\begin{gathered}
\tilde{\Delta E} \Delta E_{r}=\varepsilon_{d, 0}-\varepsilon_{f b}+\frac{\hbar^{2} k_{r}^{2}}{2 m_{d \uparrow}} \tilde{k}^{2} \\
\tilde{\Delta E}=\frac{\varepsilon_{d, 0}-\varepsilon_{f b}}{\Delta E_{r}}+\frac{\hbar^{2} k_{r}^{2}}{2 m_{d \uparrow} \Delta E_{r}} \tilde{k}^{2}
\end{gathered}
$$

From this we take

$$
\Delta E_{r}=\varepsilon_{f b}-\varepsilon_{d, 0}
$$

and

$$
k_{r}=\sqrt{\frac{2 m_{d \uparrow} \Delta E_{r}}{\hbar^{2}}}=\sqrt{\frac{2 m_{d \uparrow}\left(\varepsilon_{f b}-\varepsilon_{d, 0}\right)}{\hbar^{2}}}
$$


where we choose $\Delta E_{r}$ in this way so that it is positive (we are presuming that there will be band crossing between the $d$ and $f$ bands). In fact physically these quantities represent the difference in energy between the bands at the $X$ point and the wavevector at which the two energy bands crossover so we may in fact call $\Delta E_{r}=\Delta E_{0}$ and $k_{r}=k_{c}$. Now we see that we may write (B.2) dimensionlessly as

$$
\tilde{\Delta E}=\tilde{k}^{2}-1
$$

Looking to $E_{\mathbf{k}}$ in the same way we see

$$
\begin{gathered}
\tilde{E}_{\tilde{\mathbf{k}}} E_{r}=\sqrt{4\left(k_{r} \tilde{\mathbf{k}} \cdot \mathbf{P}\right)^{2}+\Delta E^{2}} \\
\tilde{E}_{\tilde{\mathbf{k}}}=\sqrt{4\left(\frac{k_{r} \tilde{\mathbf{k}} \cdot \mathbf{P}}{E_{r}}\right)^{2}+\left(\frac{\Delta E}{E_{r}}\right)^{2}}
\end{gathered}
$$

Now if we choose $E_{r}$ and $k_{r}$ here to be $\Delta E_{0}$ and $k_{c}$ as found before we can define a new quantity $\zeta$ such that

$$
\frac{1}{2} \zeta=\frac{P k_{c}}{\Delta E_{0}}=P \sqrt{\frac{2 m_{d \uparrow}}{\hbar^{2}\left(\varepsilon_{f b}-\varepsilon_{d, 0}\right)}}
$$

where $\mathbf{P}$ has been written so $\mathbf{P}=P \hat{\mathbf{P}}$. This now allows us to express $E_{\mathbf{k}}$ as

$$
\tilde{E}_{\tilde{\mathbf{k}}}=\sqrt{\zeta^{2}(\tilde{\mathbf{k}} \cdot \hat{\mathbf{P}})^{2}+\left(\tilde{k}^{2}-1\right)^{2}} .
$$

Putting (B.6) and (B.8) together now also gives us $\sin \alpha(\tilde{\mathbf{k}})$ :

$$
\sin \alpha(\tilde{\mathbf{k}})=\frac{1}{\sqrt{2}} \sqrt{1-\frac{\tilde{k}^{2}-1}{\sqrt{\zeta^{2}(\tilde{\mathbf{k}} \cdot \hat{\mathbf{P}})^{2}+\left(\tilde{k}^{2}-1\right)^{2}}}}
$$

We see also that $\cos \alpha(\mathbf{k})$ is also easily expressed as

$$
\cos \alpha(\tilde{\mathbf{k}})=\frac{1}{\sqrt{2}} \sqrt{1+\frac{\tilde{k}^{2}-1}{\sqrt{\zeta^{2}(\tilde{\mathbf{k}} \cdot \hat{\mathbf{P}})^{2}+\left(\tilde{k}^{2}-1\right)^{2}}}}
$$

Now we may express $\sin 2 \alpha(\mathbf{k})$ which after some simplifications can be finally written as:

$$
\sin 2 \alpha(\tilde{\mathbf{k}})=\sqrt{\frac{\zeta^{2}(\tilde{\mathbf{k}} \cdot \hat{\mathbf{P}})^{2}}{\zeta^{2}(\tilde{\mathbf{k}} \cdot \hat{\mathbf{P}})^{2}+\left(\tilde{k}^{2}-1\right)^{2}}}
$$


Now that we have $\sin 2 \alpha(\tilde{\mathbf{k}})$ and we know that $\mathbf{P}$ is in the $z$-direction we may now write

$$
\sin ^{2} 2 \alpha(\tilde{\mathbf{k}})=\frac{\zeta^{2} \tilde{k}^{2} \cos ^{2} \theta}{\zeta^{2} \tilde{k}^{2} \cos ^{2} \theta+\left(\tilde{k}^{2}-1\right)^{2}}=\sin ^{2} 2 \alpha(\tilde{k})
$$

We note that immediately the form of (B.12) is reminiscent of a Lorentzian which can be easily approximated to a delta function. By rewriting $\left(\tilde{k}^{2}-1\right)^{2}=(\tilde{k}-1)^{2}(\tilde{k}+1)^{2}$ and dividing through by $\tilde{k}^{2}$ we may write (B.12) in the following form

$$
\begin{aligned}
\sin ^{2} 2 \alpha(\tilde{k}) & =\zeta|\cos \theta| \frac{\zeta|\cos \theta|}{(\zeta|\cos \theta|)^{2}+\frac{(\tilde{k}-1)^{2}(\tilde{k}+1)^{2}}{\tilde{k}^{2}}} \\
& =\zeta|\cos \theta| \frac{\zeta|\cos \theta|}{(\zeta|\cos \theta|)^{2}+(\tilde{k}-1)^{2}\left(1+\frac{1}{\tilde{k}}\right)^{2}}
\end{aligned}
$$

By writing (B.12) in this way we see that it is remarkably close to a proper Lorentzian with an extra $\left(1+\frac{1}{\tilde{k}}\right)^{2}$ factor. If we take $\tilde{k}$ to be large then we see that $\frac{1}{\tilde{k}} \rightarrow 0$ and so (B.13) reduces to a proper Lorentzian in this limit, whereas if we take $\tilde{k}$ small then $\frac{1}{\tilde{k}} \rightarrow \infty$ which sends $\sin ^{2} 2 \alpha \rightarrow 0$. If we take $\tilde{k}$ to be around 1 then we see that $\frac{1}{\tilde{k}} \approx 1$ and so we find we will have

$$
\sin ^{2} 2 \alpha(\tilde{k}) \approx \zeta|\cos \theta| \frac{\zeta|\cos \theta|}{(\zeta|\cos \theta|)^{2}+4(\tilde{k}-1)^{2}}=\frac{1}{2} \zeta|\cos \theta| L(\tilde{k})
$$

which again is a Lorentzian with a half width of $\frac{1}{2} \Gamma=\frac{\zeta}{2}|\cos \theta|$ (called $L(\tilde{k})$ here) where $L(\tilde{k})$ has the definition:

$$
L(\tilde{k})=\frac{(\zeta|\cos \theta| / 2)}{(\zeta|\cos \theta| / 2)^{2}+(\tilde{k}-1)^{2}} .
$$

This half width is also supported from numerical calculations and increases in accuracy as $\zeta$ decreases. This can also be seen in figure B.1 where even at $\zeta|\cos \theta|=0.1$ we see very good agreement between $\sin ^{2} 2 \alpha(\tilde{k}) /(\zeta|\cos \theta| / 2)$ and $L(\tilde{k})$ around the peak and for small and large values of $\tilde{k}$. From the limiting values of $\tilde{k}$ we see that the integral is well bounded on both sides and in fact the integral $\int_{0}^{\infty} \sin ^{2} 2 \alpha(\tilde{k}) d \tilde{k}=\frac{\pi}{2}$ for any value of $\zeta|\cos \theta|$. From this we believe it is reasonable to approximate $\sin ^{2} 2 \alpha(\tilde{k})$ with

$$
\sin ^{2} 2 \alpha(\tilde{k}) \approx \frac{1}{2} \zeta|\cos \theta| \frac{2}{\pi} \delta(\tilde{k}-1)
$$

For this approximation to work we would also like the side where $\tilde{k}>1$ to become small on the same scale as it does for $\tilde{k}<1$ to keep the asymmetry of the function 


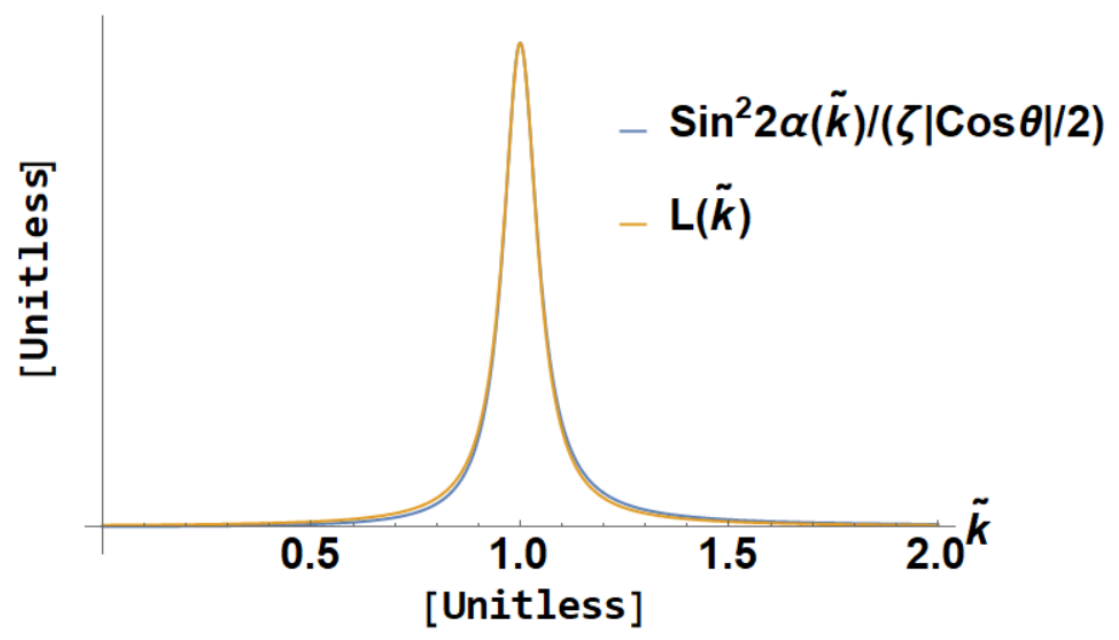

Figure B.1: Plot of $\sin ^{2} 2 \alpha(\tilde{k}) /(\zeta|\cos \theta| / 2)$ and $L(\tilde{k})$ with $\zeta|\cos \theta|=0.1$.

to a minimum and to maintain a delta function-like shape. Thus we would need

$$
(\tilde{k}-1)^{2}\left(1+\frac{1}{\tilde{k}}\right)^{2} \gg(\zeta|\cos \theta|)^{2}
$$

for $\tilde{k} \gtrsim 2$. Thus for maximal values of $|\cos \theta|$ we have the condition

$$
\zeta^{2} \ll 1
$$




\section{Bibliography}

[1] Gordon E Moore et al. Cramming more components onto integrated circuits, 1965.

[2] Thomas N Theis and H-S Philip Wong. The end of moore's law: A new beginning for information technology. Computing in Science \& Engineering, 19(2):41-50, 2017.

[3] James R Powell. The quantum limit to moore's law. Proceedings of the IEEE, 96(8):1247-1248, 2008.

[4] Franck Natali, Ben J Ruck, Natalie OV Plank, H Joe Trodahl, Simon Granville, Claire Meyer, and Walter RL Lambrecht. Rare-earth mononitrides. Progress in Materials Science, 58(8):1316-1360, 2013.

[5] E-M Anton, S Granville, A Engel, SV Chong, M Governale, U Zülicke, AG Moghaddam, HJ Trodahl, F Natali, S Vézian, et al. Superconductivity in the ferromagnetic semiconductor samarium nitride. Physical Review B, 94(2):024106, 2016.

[6] ARH Preston, S Granville, DH Housden, B Ludbrook, BJ Ruck, HJ Trodahl, A Bittar, GVM Williams, JE Downes, A DeMasi, et al. Comparison between experiment and calculated band structures for dyn and smn. Physical Review $B, 76(24): 245120,2007$.

[7] C Meyer, BJ Ruck, J Zhong, S Granville, ARH Preston, GVM Williams, and HJ Trodahl. Near-zero-moment ferromagnetism in the semiconductor smn. Physical Review B, 78(17):174406, 2008.

[8] WF Holmes-Hewett, FH Ullstad, BJ Ruck, F Natali, and HJ Trodahl. Anomalous hall effect in smn: Influence of orbital magnetism and 4 f-band conduction. Physical Review B, 98(23):235201, 2018. 
[9] Eva-Maria Anton, BJ Ruck, Claire Meyer, F Natali, Harry Warring, Fabrice Wilhelm, Andrei Rogalev, VN Antonov, and HJ Trodahl. Spin/orbit moment imbalance in the near-zero moment ferromagnetic semiconductor smn. Physical Review B, 87(13):134414, 2013.

[10] JP Dismukes, WM Yim, JJ Tietjen, and RE Novak. Vapor deposition of semiconducting mononitrides of scandium, yttrium, and the rare-earth elements. RCA Rev, 31:680-691, 1970.

[11] HA Eick, NC Baenziger, and L Eyring. The preparation, crystal structure and some properties of smn, eun and ybn1. Journal of the American Chemical Society, 78(23):5987-5989, 1956.

[12] F Natali, NOV Plank, J Galipaud, BJ Ruck, HJ Trodahl, F Semond, S Sorieul, and L Hirsch. Epitaxial growth of gdn on silicon substrate using an aln buffer layer. Journal of crystal growth, 312(24):3583-3587, 2010.

[13] Franck Natali, BJ Ruck, HJ Trodahl, S Vezian, B Damilano, Y Cordier, F Semond, Claire Meyer, et al. Role of magnetic polarons in ferromagnetic gdn. Physical Review B, 87(3):035202, 2013.

[14] MA Scarpulla, CS Gallinat, S Mack, JS Speck, and AC Gossard. Gdn (l $\left.\begin{array}{lll}1 & 1 & 1\end{array}\right)$ heteroepitaxy on gan $\left(\begin{array}{llll}0 & 0 & 0 & 1\end{array}\right)$ by n2 plasma and nh3 molecular beam epitaxy. Journal of Crystal Growth, 311(5):1239-1244, 2009.

[15] JH Richter, BJ Ruck, M Simpson, F Natali, NOV Plank, M Azeem, HJ Trodahl, ARH Preston, B Chen, James McNulty, et al. Electronic structure of eun: Growth, spectroscopy, and theory. Physical Review B, 84(23):235120, 2011.

[16] Franck Natali, Bart Ludbrook, Jules Galipaud, Natalie Plank, Simon Granville, Andrew Preston, Bin Le Do, Jan Richter, Ian Farrell, Roger Reeves, et al. Epitaxial growth and properties of gdn, eun and smn thin films. physica status solidi c, 9(3-4):605-608, 2012.

[17] Takao A Yamamoto, Takashi Nakagawa, Kengo Sako, Takayuki Arakawa, and Hiroaki Nitani. Magnetocaloric effect of rare earth mono-nitrides, tbn and hon. Journal of alloys and compounds, 376(1-2):17-22, 2004.

[18] Takashi Nakagawa, Takayuki Arakawa, Kengo Sako, Naoto Tomioka, Takao A Yamamoto, Takafumi Kusunose, Koichi Niihara, Koji Kamiya, and Takenori Numazawa. Magnetocaloric effects of ferromagnetic erbium mononitride. Journal of alloys and compounds, 408:191-195, 2006. 
[19] P Wachter, F Bommeli, L Degiorgi, P Burlet, F Bourdarot, and E Kaldis. The blue shift of the plasma edge of a ferromagnetic semimetal. Solid state communications, 105(11):675-680, 1998.

[20] S Granville, BJ Ruck, F Budde, A Koo, DJ Pringle, F Kuchler, ARH Preston, DH Housden, N Lund, A Bittar, et al. Semiconducting ground state of gdn thin films. Physical Review B, 73(23):235335, 2006.

[21] JF McNulty, BJ Ruck, and HJ Trodahl. On the ferromagnetic ground state of smn. Physical Review B, 93(5):054413, 2016.

[22] P Larson, Walter RL Lambrecht, Athanasios Chantis, and Mark van Schilfgaarde. Electronic structure of rare-earth nitrides using the lsda $+\mathrm{u}$ approach: Importance of allowing $4 \mathrm{f}$ orbitals to break the cubic crystal symmetry. Physical Review B, 75(4):045114, 2007.

[23] Giorgio L Olcese. Interconfiguration fluctuation of cerium in cen as a function of temperature and pressure. Journal of Physics F: Metal Physics, 9(3):569, 1979.

[24] Takayuki Suehiro, Naoto Hirosaki, Yoshinobu Yamamoto, Toshiyuki Nishimura, Mamoru Mitomo, Junichi Takahashi, and Hisanori Yamane. Preparation of lutetium nitride by direct nitridation. Journal of materials research, 19(3):959-963, 2004.

[25] Akira Hasegawa and Akira Yanase. Energy band structures of gd-pnictides. Journal of the Physical Society of Japan, 42(2):492-498, 1977.

[26] A Hasegawa. Electronic structure of la monopnictides. Journal of Physics C: Solid State Physics, 13(33):6147, 1980.

[27] AG Petukhov, WRL Lambrecht, and B Segall. Electronic structure of rare-earth pnictides. Physical Review B, 53(8):4324, 1996.

[28] Leonid V Pourovskii, Kris T Delaney, Chris G Van de Walle, Nicola A Spaldin, and Antoine Georges. Role of atomic multiplets in the electronic structure of rare-earth semiconductors and semimetals. Physical review letters, 102(9):096401, 2009.

[29] A Svane. Electronic structure of cerium in the self-interaction corrected local spin density approximation. Physical review letters, 72(8):1248, 1994. 
[30] CM Aerts, Paul Strange, M Horne, WM Temmerman, Zdzislawa Szotek, and Axel Svane. Half-metallic to insulating behavior of rare-earth nitrides. Physical Review B, 69(4):045115, 2004.

[31] Walter RL Lambrecht. Electronic structure and optical spectra of the semimetal scas and of the indirect-band-gap semiconductors scn and gdn. Physical Review B, 62(20):13538, 2000.

[32] Chun-gang Duan, RF Sabiryanov, Jianjun Liu, Wai-Ning Mei, Peter A Dowben, and John R Hardy. Strain induced half-metal to semiconductor transition in gdn. Physical review letters, 94(23):237201, 2005.

[33] Chun-gang Duan, RF Sabiryanov, Jianjun Liu, Wai-Ning Mei, Peter A Dowben, and John R Hardy. Theoretical study of the magnetic ordering in rareearth compounds with face-centered-cubic structure. Journal of applied physics, 97(10):10A915, 2005.

[34] Chun-Gang Duan, Renat F Sabiryanov, Wai-Ning Mei, Peter A Dowben, SS Jaswal, and Evgeny Y Tsymbal. Magnetic ordering in gd monopnictides: Indirect exchange versus superexchange interaction. Applied physics letters, 88(18):182505, 2006.

[35] Dipta Bhanu Ghosh, Molly De, and SK De. Electronic, magnetic, and optical properties of gd monopnictides: An lda $+\mathrm{u}$ study. Physical Review B, $72(4): 045140,2005$.

[36] P Larson and Walter RL Lambrecht. Electronic structure of gd pnictides calculated within the lsda $+\mathrm{u}$ approach. Physical Review B, 74(8):085108, 2006.

[37] Chandrima Mitra and Walter RL Lambrecht. Magnetic exchange interactions in the gadolinium pnictides from first principles. Physical Review B, 78(13):134421, 2008.

[38] Chandrima Mitra and Walter RL Lambrecht. Calculated interband optical transition spectra of gdn. Physical Review B, 78(19):195203, 2008.

[39] Samir Abdelouahed and M Alouani. Calculated electronic properties and structural phase transitions of gdn pnictide under hydrostatic pressure. Physical Review B, 76(21):214409, 2007.

[40] Samir Abdelouahed and M Alouani. Magnetic anisotropy in gd, gdn, and gdfe 2 tuned by the energy of gadolinium 4 f states. Physical Review B, 79(5):054406, 2009. 
[41] Athanasios N Chantis, Mark van Schilfgaarde, and Takao Kotani. Quasiparticle self-consistent g w method applied to localized $4 \mathrm{f}$ electron systems. Physical Review B, 76(16):165126, 2007.

[42] Klaus Doll. Electronic structure of gdn, and the influence of exact exchange. Journal of Physics: Condensed Matter, 20(7):075214, 2008.

[43] Martin Schlipf, Markus Betzinger, Christoph Friedrich, Marjana Ležaić, and Stefan Blügel. Hse hybrid functional within the flapw method and its application to gdn. Physical Review B, 84(12):125142, 2011.

[44] Christoph Friedrich, Markus Betzinger, Martin Schlipf, Stefan Blügel, and Arno Schindlmayr. Hybrid functionals and gw approximation in the flapw method. Journal of Physics: Condensed Matter, 24(29):293201, 2012.

[45] F Hulliger. Magnetic properties of the rare earth pnictides. Journal of Magnetism and Magnetic Materials, 8(3):183-205, 1978.

[46] F Hulliger. Rare earth pnictides. Handbook on the physics and chemistry of rare earths, 4:153-236, 1979.

[47] O Vogt and K Mattenberger. Magnetic measurements on rare earth and actinide monopnictides and monochalcogenides. Handbook on the Physics and Chemistry of Rare Earths, 17:301-407, 1993.

[48] G Busch. Magnetic properties of rare-earth compounds. Journal of Applied Physics, 38(3):1386-1394, 1967.

[49] David P Schumacher and WE Wallace. Magnetic characteristics of gadolinium, praseodymium, and thulium nitrides. Journal of Applied Physics, 36(3):984985, 1965.

[50] Lu Wang, Wai-Ning Mei, SR McHale, JW McClory, JC Petrosky, J Wu, Ratnakar Palai, Ya B Losovyj, and Peter A Dowben. Rare earth 4f hybridization with the gan valence band. Semiconductor Science and Technology, 27(11):115017, 2012.

[51] F Patthey, S Cattarinussi, W-D Schneider, Y Baer, and B Delley. Highresolution photoemission study of cen: a narrow-band material. EPL (Europhysics Letters), 2(11):883, 1986. 
[52] Yung-mau Nie and Xiao Hu. Possible half metallic antiferromagnet in a holedoped perovskite cuprate predicted by first-principles calculations. Physical review letters, 100(11):117203, 2008.

[53] Wai Kong Yeoh, Baptiste Gault, XY Cui, Chen Zhu, Michael P Moody, Liwei Li, RK Zheng, WX Li, XL Wang, SX Dou, et al. Direct observation of local potassium variation and its correlation to electronic inhomogeneity in (ba 1- $\mathrm{x}$ k x) fe 2 as 2 pnictide. Physical review letters, 106(24):247002, 2011.

[54] CV Topping, FKK Kirschner, SJ Blundell, PJ Baker, DN Woodruff, Francesca Schild, Hualei Sun, and SJ Clarke. Coexistence of magnetism and superconductivity in separate layers of the iron-based superconductor li 1- x fe x (oh) fe 1- y se. Physical Review B, 95(13):134419, 2017.

[55] I Felner, U Asaf, Y Levi, and O Millo. Coexistence of magnetism and superconductivity in $\mathrm{r} 1.4$ ce 0.6 rusr 2 cu 2 o $10-\delta \mathrm{s}(\mathrm{r}=\mathrm{eu}$ and $\mathrm{gd})$. Physical Review B, 55(6):R3374, 1997.

[56] Vladimir P Mineev, K Samokhin, and LD Landau. Introduction to unconventional superconductivity. CRC Press, 1999.

[57] VP Mineev and T Champel. Theory of superconductivity in ferromagnetic superconductors with triplet pairing. Physical Review B, 69(14):144521, 2004.

[58] The rise of quantum materials. Nat. Phys., 12:105, 2016.

[59] H Kamerlingh Onnes. Further experiments with liquid helium. In Proceedings of the KNAW, volume 13, pages 1910-1911, 1911.

[60] John Bardeen, Leon N Cooper, and John Robert Schrieffer. Theory of superconductivity. Physical review, 108(5):1175, 1957.

[61] P Dai, BC Chakoumakos, GF Sun, KW Wong, Y Xin, and DF Lu. Synthesis and neutron powder diffraction study of the superconductor hgba2ca2cu3o8+ $\delta$ by tl substitution. Physica C: Superconductivity, 243(3-4):201-206, 1995.

[62] Leon N Cooper. Bound electron pairs in a degenerate fermi gas. Physical Review, 104(4):1189, 1956.

[63] J George Bednorz and K Alex Müller. Possible hight c superconductivity in the ba- la- cu- o system. Zeitschrift für Physik B Condensed Matter, 64(2):189-193, 1986. 
[64] Pia Jensen Ray. Figure 2.4 in master's thesis, "structural investigation of $\mathrm{la}_{2-x} \mathrm{Sr}_{x} \mathrm{cuO}_{4+y}$ - following staging as a function of temperature". Master's thesis, Niels Bohr Institute, Faculty of Science, University of Copenhagen, Copenhagen, Denmark, 2015. DOI: 10.6084/m9.figshare.2075680.v2.

[65] NE Bickers, DJ Scalapino, and RT Scalettar. Cdw and sdw mediated pairing interactions. International Journal of Modern Physics B, 1(03n04):687-695, 1987.

[66] Philip W Anderson. The resonating valence bond state in la2cuo4 and superconductivity. science, 235(4793):1196-1198, 1987.

[67] Gabriel Kotliar and Jialin Liu. Superexchange mechanism and d-wave superconductivity. Physical Review B, 38(7):5142, 1988.

[68] Ming Shi, Johan Chang, Stephane Pailhés, MR Norman, JC Campuzano, Martin Månsson, Thomas Claesson, Oscar Tjernberg, Azzedin Bendounan, Luc Patthey, et al. Coherent d-wave superconducting gap in underdoped la 2- x sr $\mathrm{x}$ cuo 4 by angle-resolved photoemission spectroscopy. Physical review letters, 101(4):047002, 2008.

[69] Jianqiao Meng, Wentao Zhang, Guodong Liu, Lin Zhao, Haiyun Liu, Xiaowen Jia, Wei Lu, Xiaoli Dong, Guiling Wang, Hongbo Zhang, et al. Monotonic dwave superconducting gap of the optimally doped bi 2 sr 1.6 la 0.4 cuo 6 superconductor by laser-based angle-resolved photoemission spectroscopy. Physical Review B, 79(2):024514, 2009.

[70] Yasumasa Tsutsumi, Kazushige Machida, Tetsuo Ohmi, and Masa-aki Ozaki. A spin triplet superconductor upt3. Journal of the Physical Society of Japan, 81(7):074717, 2012.

[71] Yoshiteru Maeno, Shunichiro Kittaka, Takuji Nomura, Shingo Yonezawa, and Kenji Ishida. Evaluation of spin-triplet superconductivity in sr2ruo4. Journal of the Physical Society of Japan, 81(1):011009, 2011.

[72] Jun Nagamatsu, Norimasa Nakagawa, Takahiro Muranaka, Yuji Zenitani, and Jun Akimitsu. Superconductivity at $39 \mathrm{k}$ in magnesium diboride. nature, 410(6824):63-64, 2001.

[73] Hyoung Joon Choi, David Roundy, Hong Sun, Marvin L Cohen, and Steven G Louie. The origin of the anomalous superconducting properties of mgb 2. $\mathrm{Na}$ ture, 418(6899):758-760, 2002. 
[74] Yoichi Kamihara, Takumi Watanabe, Masahiro Hirano, and Hideo Hosono. Iron-based layered superconductor la $[\mathrm{o} 1-\mathrm{x} \mathrm{f} \mathrm{x}]$ feas $(\mathrm{x}=0.05-0.12)$ with $\mathrm{t} \mathrm{c}=$ 26 k. Journal of the American Chemical Society, 130(11):3296-3297, 2008.

[75] Hideo Hosono and Kazuhiko Kuroki. Iron-based superconductors: Current status of materials and pairing mechanism. Physica C: Superconductivity and its Applications, 514:399-422, 2015.

[76] Yew San Hor, Anthony J Williams, Joseph G Checkelsky, Pedram Roushan, Jungpil Seo, Qiang Xu, Henny W Zandbergen, Ali Yazdani, Nai Phuan Ong, and Robert Joseph Cava. Superconductivity in cu x bi 2 se 3 and its implications for pairing in the undoped topological insulator. Physical review letters, 104(5):057001, 2010.

[77] Liang Fu and Erez Berg. Odd-parity topological superconductors: theory and application to cu x bi 2 se 3. Physical review letters, 105(9):097001, 2010.

[78] Masatoshi Sato and Yoichi Ando. Topological superconductors: a review. Reports on Progress in Physics, 80(7):076501, 2017.

[79] Yasuhiro Asano, Akihiro Sasaki, and Alexander A Golubov. Dirty twoband superconductivity with interband pairing order. New journal of physics, 20(4):043020, 2018.

[80] Governale M. Mogheddam, A. G. and U. Züelicke. Theory of spinless $p$-wave superconductivity. 2015.

[81] Manfred Sigrist and Kazuo Ueda. Phenomenological theory of unconventional superconductivity. Reviews of Modern physics, 63(2):239, 1991.

[82] Lok C Lew Yan Voon and Morten Willatzen. The kp method: electronic properties of semiconductors. Springer Science \& Business Media, 2009.

[83] YU Peter and Manuel Cardona. Fundamentals of semiconductors: physics and materials properties. Springer Science \& Business Media, 2010.

[84] Dr Helmut Föll. 2.1.5 band structures and structural representation. https: //www.tf.uni-kiel.de/matwis/amat/semi_en/index.html, 2019. [Online; accessed 19-February-2020].

[85] Wikimedia Commons. File:brillouin zone (1st, fcc).svg — wikimedia commons, the free media repository. https://commons.wikimedia.org/w/index.php? 
title=File:Brillouin_Zone_(1st,_FCC).svg\&oldid=351404377, 2019. [Online; accessed 19-February-2020].

[86] Andres Ellens, H Andres, MLH Ter Heerdt, RT Wegh, A Meijerink, and G Blasse. Spectral-line-broadening study of the trivalent lanthanide-ion series. ii. the variation of the electron-phonon coupling strength through the series. Physical Review B, 55(1):180, 1997.

[87] Tawinan Cheiwchanchamnangij. Applications of the Quasiparticle Selfconsistent GW Method. PhD thesis, Case Western Reserve University, 2014.

[88] Narayan N Som, Venu H Mankad, Shweta D Dabhi, Anjali Patel, and Prafulla K Jha. Magnetic behavior study of samarium nitride using density functional theory. Journal of Magnetism and Magnetic Materials, 448:186-191, 2018.

[89] WF Holmes-Hewett, RG Buckley, BJ Ruck, F Natali, and HJ Trodahl. Optical spectroscopy of smn: Evidence for $4 \mathrm{f}$ transport. arXiv preprint arXiv:1903.05326, 2019.

[90] C Morari, F Beiuşeanu, Igor Di Marco, L Peters, E Burzo, S Mican, and L Chioncel. Magnetism and electronic structure calculation of smn. Journal of Physics: Condensed Matter, 27(11):115503, 2015.

[91] Paolo Marconcini and Massimo Macucci. The kp method and its application to graphene, carbon nanotubes and graphene nanoribbons: The dirac equation. arXiv preprint arXiv:1105.1351, 2011.

[92] Piers Coleman. Heavy fermions: Electrons at the edge of magnetism. Handbook of magnetism and advanced magnetic materials, 2007.

[93] Charles Kittel and Ching-yao Fong. Quantum theory of solids, volume 5. Wiley New York, 1963.

[94] N Kristoffel, P Konsin, and T Örd. Two-band model for high-temperature superconductivity. La Rivista del Nuovo Cimento (1978-1999), 17(9):1-41, 1994.

[95] AI Akhiezer and IA Akhiezer. On the coexistence of superconductivity and ferromagnetism. SOVIET PHYSICS JETP, 16(6), 1963. 